\title{
LIMIT THEOREMS FOR BESSEL AND DUNKL PROCESSES OF LARGE DIMENSIONS AND FREE CONVOLUTIONS
}

\author{
MICHAEL VOIT, JEANNETTE H.C. WOERNER
}

\begin{abstract}
We study Bessel and Dunkl processes $\left(X_{t, k}\right)_{t \geq 0}$ on $\mathbb{R}^{N}$ with possibly multivariate coupling constants $k \geq 0$. These processes describe interacting particle systems of Calogero-Moser-Sutherland type with $N$ particles. For the root systems $A_{N-1}$ and $B_{N}$ these Bessel processes are related with $\beta$-Hermite and $\beta$-Laguerre ensembles. Moreover, for the frozen case $k=\infty$, these processes degenerate to deterministic or pure jump processes.

We use the generators for Bessel and Dunkl processes of types A and B and derive analogues of Wigner's semicircle and Marchenko-Pastur limit laws for $N \rightarrow \infty$ for the empirical distributions of the particles with arbitrary initial empirical distributions by using free convolutions. In particular, for Dunkl processes of type B new non-symmetric semicircle-type limit distributions on $\mathbb{R}$ appear. Our results imply that the form of the limiting measures is already completely determined by the frozen processes. Moreover, in the frozen cases, our approach leads to a new simple proof of the semicircle and MarchenkoPastur limit laws for the empirical measures of the zeroes of Hermite and Laguerre polynomials respectively.
\end{abstract}

\section{INTRODUCTION}

Calogero-Moser-Sutherland particle systems on $\mathbb{R}$ or $[0, \infty[$ with $N$ particles can be described as multivariate Bessel processes on closed Weyl chambers in $\mathbb{R}^{N}$. These Bessel processes are time-homogeneous diffusions with well-known transition probabilities and generators of the transition semigroups; moreover they are solution of the associated stochastic differential equations (SDEs); see [CGY, GY, R1, R2, RV1, RV2, DV, An] for the background. These multivariate Bessel processes $\left(X_{t, k}\right)_{t \geq 0}$ depend on their starting configurations for $t=0$, root systems, and a possibly multidimensional multiplicity parameter $k$ which describes the strength of interaction of the particles to each other and to the boundary.

Furthermore, based on the theory of Dunkl operators, these Bessel processes on Weyl chambers in $\mathbb{R}^{N}$ can be extended in a canonical way to Feller processes $\left(X_{t, k}\right)_{t>0}$ on $\mathbb{R}^{N}$ by adding random reflections which are associated with the underlying root systems and multiplicity parameters $k$; see [CGY, GY, R1, RV1, RV2 for the background. These diffusion-reflection processes are called Dunkl processes; for the background in analysis and mathematical physics see [R2, An, DV] and

Date: September 30, 2020.

2010 Mathematics Subject Classification. Primary 60F05; Secondary 60F15, 60B20, 60J60, $60 \mathrm{~K} 35,70 \mathrm{~F} 10,82 \mathrm{C} 22$.

Key words and phrases. Bessel processes, Dunkl processes, interacting particle systems, Calogero-Moser-Sutherland models, zeroes of Hermite polynomials, zeroes of Laguerre polynomials, $\beta$-Hermite ensembles, $\beta$-Laguerre ensembles, Dyson Brownian motion, semicircle laws, Marchenko-Pastur laws, free convolution, Stieltjes transform, Burgers equation. 
references there. For these Bessel and Dunkl processes $\left(X_{t, k}=\left(X_{t, k}^{1}, \ldots, X_{t, k}^{N}\right)\right)_{t \geq 0}$ we derive limit theorems for the empirical distributions

$$
\frac{1}{N}\left(\delta_{X_{t, k}^{1} / \sqrt{N}}+\ldots+\delta_{X_{t, k}^{N} / \sqrt{N}}\right)
$$

of the $N$ particles as $N \rightarrow \infty$ for $t>0$ under the condition that these empirical distributions converge for $t=0$ and $N \rightarrow \infty$ weakly to some given probability measure $\mu \in M^{1}(\mathbb{R})$ which satisfies some moment condition. We prove that then the measures in 1.1 converge a.s. weakly to probability measures $\mu_{t} \in M^{1}(\mathbb{R})$ which can be described in terms of $\mu$ and free additive convolutions $\boxplus$. The appearance of free probability is not surprising, as for some root systems and multiplicities $k$, our Bessel processes describe the evolutions of spectra of classical random matrix models like the $\beta$-Hermite and $\beta$-Laguerre ensembles of Dumitriu and Edelman DE1, DE2. Thus our results are closely related to Wigner's semicircle laws and Marchenko-Pastur limit laws in different random matrix settings; see e.g. [AGZ, $\mathrm{D}, \mathrm{HT}, \mathrm{Me}, \mathrm{NS}, \mathrm{OP}, \mathrm{RS}$. We mention that in particular the dynamic approach in Section 4.3 of AGZ is closely related to our paper. However, our approach via moments is simpler than that in [AGZ] in view of the technical tools on stochastic processes. Moreover, in AGZ only processes of type A are considered.

It is clear that for our limit theorems we need some control on the parameters $k$ and the types of root systems which must exist for all dimensions $N$. This and the need of nontrivial interactions of the particles are the reason that we will restrict our attention to the root systems of types $A_{N-1}$ and $B_{N}$ on $\mathbb{R}^{N}$. Moreover, as the processes for the root systems $D_{N}$ differ from those for $B_{N}$ only in the behavior of one extremal particle (with a suitable relation between the multiplicities; see e.g. AV1, V]), our results on the empirical distributions of $N$ particles for $N \rightarrow \infty$ for the root systems $D_{N}$ may be easily regarded as a special case of some $B_{N}$-case.

We next briefly summarize some details of the main results of this paper.

For the root systems $A_{N-1}$, we fix a multiplicity $\left.k \in\right] 0, \infty[$. The associated Bessel processes $\left(X_{t, k}\right)_{t \geq 0}$ then live on the closed Weyl chambers

$$
C_{N}^{A}:=\left\{x \in \mathbb{R}^{N}: \quad x_{1} \geq x_{2} \geq \ldots \geq x_{N}\right\},
$$

and the generators of the transition semigroups are

$$
\mathcal{L}_{k} f:=\frac{1}{2} \Delta f+k \sum_{i=1}^{N}\left(\sum_{j \neq i} \frac{1}{x_{i}-x_{j}}\right) \frac{\partial}{\partial x_{i}} f,
$$

where we assume reflecting boundaries, i.e., the domain of $\mathcal{L}_{k}$ is

$D\left(\mathcal{L}_{k}\right):=\left\{\left.f\right|_{C_{N}^{A}}: f \in C^{(2)}\left(\mathbb{R}^{N}\right), f\right.$ invariant under all coordinate permutations $\}$.

It will be convenient, also to consider the renormalized processes $\left(\tilde{X}_{t, k}:=\frac{X_{t, k}}{\sqrt{k}}\right)_{t \geq 0}$, which satisfy the SDEs

$$
d \tilde{X}_{t, k}^{i}=\frac{1}{\sqrt{k}} d B_{t}^{i}+\sum_{j \neq i} \frac{1}{\tilde{X}_{t, k}^{i}-\tilde{X}_{t, k}^{j}} d t \quad(i=1, \ldots, N)
$$

with $N$-dimensional Brownian motions $\left(B_{t}^{1}, \ldots, B_{t}^{N}\right)_{t \geq 0}$. We mention that these SDEs admit unique strong solutions by GrM] even if these SDEs do not satisfy the standard assumptions for general SDEs as e.g. in the monograph $[\mathrm{P}]$ due to the 
singularities on the boundary. For $k=\infty$ these SDEs degenerate to the ODEs

$$
\frac{d}{d t} \tilde{X}_{t, \infty}^{i}=\sum_{j \neq i} \frac{1}{\tilde{X}_{t, \infty}^{i}-\tilde{X}_{t, \infty}^{j}} \quad(i=1, \ldots, N) .
$$

For the root systems $B_{N}$, we have $\left.k=\left(k_{1}, k_{2}\right) \in\right] 0, \infty\left[{ }^{2}\right.$, the Bessel processes live on

$$
C_{N}^{B}:=\left\{x \in \mathbb{R}^{N}: \quad x_{1} \geq x_{2} \geq \ldots \geq x_{N} \geq 0\right\},
$$

and the generators are

$$
\mathcal{L}_{k} f:=\frac{1}{2} \Delta f+k_{2} \sum_{i=1}^{N} \sum_{j \neq i}\left(\frac{1}{x_{i}-x_{j}}+\frac{1}{x_{i}+x_{j}}\right) \frac{\partial}{\partial x_{i}} f \quad+k_{1} \sum_{i=1}^{N} \frac{1}{x_{i}} \frac{\partial}{\partial x_{i}} f,
$$

where we again assume reflecting boundaries. We now write the multiplicities as $k=\left(k_{1}, k_{2}\right)=(\nu \cdot \beta, \beta)$ with $\nu \geq 0, \beta>0$. Moreover, we study the renormalized Bessel processes $\left(\tilde{X}_{t, k}:=X_{t, k} / \sqrt{\beta}\right)_{t \geq 0}$ which then satisfy the SDEs

$$
d \tilde{X}_{t, k}^{i}=\frac{1}{\sqrt{\beta}} d B_{t}^{i}+\left(\sum_{j \neq i} \frac{\tilde{X}_{t, k}^{i}}{\left(\tilde{X}_{t, k}^{i}\right)^{2}-\left(\tilde{X}_{t, k}^{j}\right)^{2}}+\frac{\nu}{\tilde{X}_{t, k}^{i}}\right) d t \quad(i=1, \ldots, N)
$$

with $\left(B_{t}^{1}, \ldots, B_{t}^{N}\right)_{t \geq 0}$ as above. For $\beta=\infty$ these SDEs degenerate to the ODEs

$$
\frac{d}{d t} \tilde{X}_{t, \infty}^{i}=\sum_{j \neq i} \frac{\tilde{X}_{t, \infty}^{i}}{\left(\tilde{X}_{t, \infty}^{i}\right)^{2}-\left(\tilde{X}_{t, \infty}^{j}\right)^{2}}+\frac{\nu}{\tilde{X}_{t, \infty}^{i}} \quad(i=1, \ldots, N) .
$$

We point out that the limit transitions $k, \beta \rightarrow \infty$ above for the root systems of type A and B lead to interesting limit theorems which admit interpretations for $\beta$-random matrix ensembles; see [DE2, AHV, AKM1, AKM2, AV1, AV2, GK, GM, V, VW1.

We next recapitulate from [R1, R2, RV1, RV2 that for the root systems $A_{N-1}$ and $B_{N}$, the transition probabilities of the Bessel processes $\left(X_{t, k}\right)_{t \geq 0}$ have the form

$$
K_{t}(x, A)=c_{k} \int_{A} \frac{1}{t^{\gamma_{k}+N / 2}} e^{-\left(\|x\|^{2}+\|y\|^{2}\right) /(2 t)} J_{k}\left(\frac{x}{\sqrt{t}}, \frac{y}{\sqrt{t}}\right) \cdot w_{k}(y) d y
$$

for $t>0, x \in C_{N}$, and $A \subset C_{N}$ a Borel set (with $C_{N}=C_{N}^{A}, C_{N}^{B}$ respectively), with the weight functions

$$
w_{k}^{A}(x):=\prod_{i<j}\left(x_{i}-x_{j}\right)^{2 k}, \quad w_{k}^{B}(x):=\prod_{i<j}\left(x_{i}^{2}-x_{j}^{2}\right)^{2 k_{2}} \cdot \prod_{i=1}^{N} x_{i}^{2 k_{1}},
$$

and with the constants $\gamma_{k}^{A}(k):=k N(N-1) / 2$ and $\gamma_{k}^{B}\left(k_{1}, k_{2}\right):=k_{2} N(N-1)+k_{1} N$ respectively. In both cases, $w_{k}$ is homogeneous of degree $2 \gamma_{k}, c_{k}>0$ is a known normalization. $J_{k}$ is a multivariate Bessel function of type $A_{N-1}$ or $B_{N}$ with multiplicities $k$ or $\left(k_{1}, k_{2}\right)$ which is analytic on $\mathbb{C}^{N} \times \mathbb{C}^{N}$ with $J_{k}(x, y)>0$ for $x, y \in \mathbb{R}^{N}$. Moreover, $J_{k}(x, y)=J_{k}(y, x)$ and $J_{k}(0, y)=1$ for $x, y \in \mathbb{C}^{N}$; see e.g. [R1, R2]. In particular, if $X_{0, k}=0$, then $X_{t, k}$ has the Lebesgue density

$$
\frac{c_{k}}{t^{\gamma+N / 2}} e^{-\|y\|^{2} /(2 t)} \cdot w_{k}(y)
$$

on $C_{N}$ for $t>0$. Hence, for the root systems $A_{N-1}$ and $B_{N}$, the processes $\left(X_{t, k}\right)_{t \geq 0}$ are related to $\beta$-Hermite and $\beta$-Laguerre ensembles in DE1].

We now turn to the main results of this paper. 
The following result in the A-case for $k=\infty$ is a special case of the main result of Section 2. It uses the classical free additive convolution $\boxplus$ and the semicircle distributions $\mu_{s c, R}$ with supports $[-R, R]$ for $R \geq 0$ as discussed e.g. in [AGZ, NS].

Theorem 1.1. Let $\mu \in M^{1}(\mathbb{R})$ be a probability measure with compact support, and let $\left(x_{N, n}\right)_{N \geq 1,1 \leq n \leq N} \subset \mathbb{R}$ a sequence with $x_{N, n-1} \geq x_{N, n}$ for $2 \leq n \leq N$ such that the normalized empirical measures

$$
\mu_{N, 0}:=\frac{1}{N}\left(\delta_{x_{N, 1} / \sqrt{N}}+\ldots \delta_{x_{N, N} / \sqrt{N}}\right)
$$

tend weakly to $\mu$ for $N \rightarrow \infty$. If we take the solutions $\left(\phi_{N, 1}(t), \ldots, \phi_{N, N}(t)\right)$ of 1.4) with $\phi_{N, n}(0)=x_{N, n}$ and the associated normalized empirical measures

$$
\mu_{N, t}:=\frac{1}{N}\left(\delta_{\phi_{N, 1}(t) / \sqrt{N}}+\ldots+\delta_{\phi_{N, N}(t) / \sqrt{N}}\right) \quad(t \geq 0)
$$

for $N \in \mathbb{N}$, then for each $t \in\left[0, \infty\left[\right.\right.$, the $\mu_{N, t}$ tend weakly to $\mu_{s c, 2 \sqrt{t}} \boxplus \mu$.

The proof of this result will be based on recursive formulas for the moments of the measures $\mu_{N, t}$ which follow from (1.4). By using the Stieltjes and R-transforms of the measures $\mu_{N, t}$ and their limits, we shall see that the limits of the $\mu_{N, t}$ are equal to $\mu_{s c, 2 \sqrt{t}} \boxplus \mu$. We mention that this ODE-approach includes a classical limit result on the empirical distributions of the zeroes of the classical Hermite polynomials $H_{N}$ for $N \rightarrow \infty$; see Corollary 2.7 below. For other proofs of this result see e.g. D, G, KM1.

In Section 3 we use standard techniques from probability like the BurkholderDavis-Gundy inequality and the lemma of Borel-Cantelli to extend the results for $k=\infty$ to a.s. results for Bessel processes of type A for finite multiplicities $k$ :

Theorem 1.2. Let $\mu \in M^{1}(\mathbb{R})$ be a probability measure with compact support, and let $\left(x_{N, n}\right)_{N \geq 1,1 \leq n \leq N} \subset \mathbb{R}$ with $x_{N, n-1} \geq x_{N, n}$ for $2 \leq n \leq N$ such that the measures in (1.11) tend weakly to $\mu$ for $N \rightarrow \infty$.

For $k \geq 1 / 2$ and $N \in \mathbb{N}$, consider the renormalized Bessel processes $\left(\tilde{X}_{t, k}\right)_{t \geq 0}$ with start in $\left(x_{N, 1}, \ldots, x_{N, n}\right) \in C_{N}^{A}$. Then, for $t \geq 0$, the empirical measures

$$
\tilde{\mu}_{N, t}:=\frac{1}{N}\left(\delta_{\tilde{X}_{t, k}^{1} / \sqrt{N}}+\ldots+\delta_{\tilde{X}_{t, k}^{N} / \sqrt{N}}\right) \text {. }
$$

tend weakly to $\mu_{s c, 2 \sqrt{t}} \boxplus \mu$ a.s. for $N \rightarrow \infty$.

We now turn to the main results for the case B in the Sections 4 and 5 . The first result is analogous to Theorem 1.1

Theorem 1.3. Let $\mu \in M^{1}([0, \infty[)$ be a probability measure with compact support. Let $\left(x_{N, n}\right)_{N \geq 1,1 \leq n \leq N} \subset\left[0, \infty\left[\right.\right.$ with $\left(x_{N, 1}, \ldots, x_{N, N}\right) \in C_{N}^{B}$ such that the measures

$$
\mu_{N, 0}:=\frac{1}{N}\left(\delta_{x_{N, 1} / \sqrt{2 N}}+\ldots \delta_{x_{N, N} / \sqrt{2 N}}\right)
$$

tend weakly to $\mu$ for $N \rightarrow \infty$. Consider the solutions $\phi_{N}$ of (1.7) with start in $\left(x_{N, 1}, \cdots, x_{N, N}\right)$. If

$$
\lim _{N \rightarrow \infty} \nu(N) / N=: \nu_{0} \geq 0
$$

then for each $t \in[0, \infty[$, the empirical measures

$$
\mu_{N, t}:=\frac{1}{N}\left(\delta_{\frac{\phi_{N, 1}(t)}{\sqrt{2 N}}}+\ldots+\delta_{\frac{\phi_{N, N}(t)}{\sqrt{2 N}}}\right) \quad(t \geq 0)
$$


tend weakly to $\sqrt{\mu_{M P, \nu_{0}, t} \boxplus\left(\mu_{s c, 2 \sqrt{t}} \boxplus \mu_{\text {even }}\right)^{2}}$ where the symbols $\sqrt{\cdot}$ and.$^{2}$ mean push forwards of probability measures under these mappings, $\mu_{\text {even }}$ is the even part of $\mu$, and the measures $\mu_{M P, \nu_{0}, t}$ are Marchenko-Pastur distributions with parameters $\nu_{0}, t$.

Again, this ODE-approach leads to a classical limit result on the empirical distributions of the zeroes of the classical Laguerre polynomials $L_{N}^{(\alpha)}$ for $N \rightarrow \infty$ in Section 4; see also G, KM2 and references there for other proofs of these facts. Moreover, this result admits the following extension:

Theorem 1.4. Let $\mu \in M^{1}([0, \infty[)$ be a probability measure with compact support. Let $\left(x_{N, n}\right)_{N \geq 1,1 \leq n \leq N} \subset\left[0, \infty\left[\right.\right.$ with $\left(x_{N, 1}, \ldots, x_{N, N}\right) \in C_{N}^{B}$ such that the measures in (1.13) tend weakly to $\mu$. Consider the normalized Bessel processes $\left(\tilde{X}_{t, k}\right)_{t \geq 0}$ of type $B$ with start in $\left(x_{N, 1}, \ldots, x_{N, N}\right) \in C_{N}^{B}$. Then, for each $t \geq 0$, and $\lim _{N \rightarrow \infty} \nu(N) / N=: \nu_{0} \geq 0$, the measures

$$
\mu_{N, t}:=\frac{1}{N}\left(\delta_{\frac{\tilde{X}_{t, k}^{1}}{\sqrt{2 N}}}+\ldots+\delta_{\frac{\tilde{X}_{t, k}^{N}}{\sqrt{2 N}}}\right)
$$

tend a.s. weakly to $\sqrt{\mu_{M P, \nu_{0}, t} \boxplus\left(\mu_{s c, 2 \sqrt{t}} \boxplus \mu_{\text {even }}\right)^{2}}$.

The description of the limits in Theorems 1.3 and 1.4 seems to be new; a partial result on the PDEs of the Stieltjes transforms of the limits can be found in CG].

Finally, in Sections 6-8 we turn to Dunkl processes. For the root systems $A_{N-1}$, the Dunkl processes differ from the corresponding Bessel processes only by additional permutations of particles. As these permutation have no influence to the limit theorems 1.1 and 1.2 the transition from Bessel to Dunkl processes leads to the same results; we thus do not study this case.

However, for root systems of type $B$, the transition from Bessel to Dunkl processes leads to additional random sign-changes of all particles even in the freezing case $\beta=\infty$ and thus to new effects. To explain the main results, we first recapitulate some notations. We fix some multiplicity $k=\left(k_{1}, k_{2}\right) \in\left[0, \infty\left[^{2}\right.\right.$ for the root system $B_{N}$ and write these constants as $\left(k_{1}, k_{2}\right)=(\beta, \nu \beta)$ with $\beta>0$ and $\nu \geq 0$ as above. By [RV1, RV2, CGY], the associated renormalized Dunkl processes $\left(\tilde{X}_{t, \nu, \beta}\right)_{t \geq 0}$ on $\mathbb{R}^{N}$ are then defined as Feller processes on $\mathbb{R}^{N}$ with the generators

$$
\tilde{\mathcal{L}}_{k_{0}, \beta} u(x):=\frac{1}{2 \beta} \Delta u(x)+L_{\nu} u(x)
$$

for $u \in C_{c}^{2}\left(\mathbb{R}^{N}\right)$ where

$$
\begin{aligned}
L_{\nu} u(x) & :=\sum_{i=1}^{N}\left(\sum_{j: j \neq i} \frac{2 x_{i}}{x_{i}^{2}-x_{j}^{2}}+\frac{\nu}{x_{i}}\right) u_{x_{i}}(x)+\frac{\nu}{2} \sum_{i=1}^{N} \frac{u\left(\sigma_{i} x\right)-u(x)}{x_{i}^{2}} \\
& +\frac{1}{2} \sum_{i, j: j \neq i}\left(\frac{u\left(\sigma_{i, j} x\right)-u(x)}{\left(x_{i}-x_{j}\right)^{2}}+\frac{u\left(\sigma_{i, j}^{-} x\right)-u(x)}{\left(x_{i}+x_{j}\right)^{2}}\right)
\end{aligned}
$$

is, by definition, the generator of the frozen process with $\beta=\infty . \sigma_{i}, \sigma_{i, j}, \sigma_{i, j}^{-}(i \neq j)$ denote reflections on $\mathbb{R}^{N}$ where $\sigma_{i}$ changes the sign of the $i$-th coordinate, $\sigma_{i, j}$ exchanges the coordinates $i, j$, and $\sigma_{i, j}^{-}$exchanges the coordinates $i, j$ and changes the signs of these coordinates in addition. 
If the starting measure $\mu \in M^{1}(\mathbb{R})$ of a sequence of such renormalized Dunkl processes is symmetric, then we may choose the starting sequences $\left(x_{N, n}\right)_{N \geq 1,1 \leq n \leq N} \subset$ $\mathbb{R}$ as e.g. in Theorem 1.3 in a symmetric way, and symmetry arguments lead to symmetric extensions of the Marchenko-Pastur limit theorems 1.3 and 1.4

However, for non-symmetric starting configurations, completely new limit distributions appear. We study the analytic part of this problem in Section 7 for the frozen case where we describe the even parts of the limit measures via Theorem 1.3 and 1.4. while the odd parts are described via their Stieltjes transforms. For this we shall first derive linear PDEs for the Stieltjes transforms of the odd parts, and then we shall deduce these Stieltjes transforms in an explicit way. Unfortunately, we are not able to describe the associated probability measures via free convolutions in general. However, for the case $\lim _{N \rightarrow \infty} \nu(N) / N=\nu_{0}=0$ and a quarter circle distribution on $[0,2]$ as starting measure, we are able to compute the associated measures for all times $t \geq 0$ in an explicit way; see Example 7.6. After this analytic part in Section 7 on the frozen case, we extend Theorem 1.4 to renormalized Dunkl processes $\left(\tilde{X}_{t, \nu, \beta}\right)_{t \geq 0}$ in Section 8 .

\section{A sequence of ODEs and the Semicircle laW}

In this section we study a sequence of ODEs with $N \geq 2$ equations which are closely related to the zeroes of the Hermite polynomials $H_{N}$. We show that the empirical distributions of the $N$-dimensional solutions of these ODEs for $N \rightarrow \infty$ are related to the semicircle law. We identify the limits as free additive convolution of the semicircle law with the law associated to the starting value. As a special case this leads to the well-known semicircle law for the empirical distributions of the zeroes of $H_{N}$. Let us start with the ODEs:

The ODE 2.1. Let $N \geq 2$. On the interior of the closed Weyl chamber

$$
C_{N}^{A}:=\left\{x \in \mathbb{R}^{N}: \quad x_{1} \geq x_{2} \geq \ldots \geq x_{N}\right\} \subset \mathbb{R}^{N}
$$

of type $\mathrm{A}$, consider the $\mathbb{R}^{N}$-valued function

$$
H(x):=\left(\sum_{j \neq 1} \frac{1}{x_{1}-x_{j}}, \ldots, \sum_{j \neq N} \frac{1}{x_{N}-x_{j}}\right) .
$$

It is shown in [VW2] that for each initial condition $x_{0} \in C_{N}^{A}$, the ODE

$$
\frac{d x}{d t}(t)=H(x(t)), \quad x(0)=x_{0}
$$

has a unique solution for all $t \geq 0$ in the sense that $\left[0, \infty\left[\rightarrow C_{N}^{A}, t \mapsto x(t)\right.\right.$ is continuous such that $x(t)$ is in the interior of $C_{N}^{A}$ and solves the ODE in (2.1) for $t>0$. For the ODE (2.1) we also refer to AV1, VW1. We denote solutions of the ODE (2.1) by $\phi_{N}:=\left(\phi_{N, 1}, \ldots, \phi_{N, N}\right)$ where we suppress the dependence on $x_{0}$.

For $x_{0}=0 \in C_{N}^{A}$, the solution of 2.1 can be expressed via the zeroes of the Hermite polynomial $H_{N}$ where, as usual, the $\left(H_{N}\right)_{N \geq 0}$ are orthogonal w.r.t. the density $e^{-x^{2}}$ on $\mathbb{R}$ as e.g. in $[\mathrm{Sz}$. For this we need the following fact due to Stieltjes; see Section 6.7 of $[\mathrm{Sz}$ ] or $\mathrm{AKM} 1$ :

Lemma 2.2. Let $z \in C_{N}^{A}$. Then $z:=\left(z_{1}, \ldots, z_{N}\right)$ consists of the ordered zeroes of $H_{N}$ if and only if

$$
z_{i}=\sum_{j: j \neq i} \frac{1}{z_{i}-z_{j}} \quad \text { for } \quad i=1, \ldots, N .
$$


Lemma 2.2 immediately implies the following result; see AV1:

Corollary 2.3. Let $z \in C_{N}^{A}$ as above and $c \geq 0$. Then $\phi_{N}(t)=\sqrt{2 t+c^{2}} \cdot z$ is a solution of (2.1).

We now turn to the empirical measures of solutions of 2.1). We choose starting sequences $\left(x_{N, k}\right)_{1 \leq k \leq N} \subset \mathbb{R}$ with $\left(x_{N, 1}, \ldots, x_{N, N}\right) \in C_{N}^{A}$ such that for each $N$ the ODE (2.1) has a solution with start in $\left(x_{N, 1}, \ldots, x_{N, N}\right)$. For $t \geq 0$ consider the associated solutions $\phi_{N}(t)$ and normalized empirical measures

$$
\mu_{N, t}:=\frac{1}{N}\left(\delta_{\phi_{N, 1}(t) / \sqrt{N}}+\ldots+\delta_{\phi_{N, N}(t) / \sqrt{N}}\right) \in M^{1}(\mathbb{R}) .
$$

The aim of this section is to characterize the limiting empirical measures $\mu_{t}$ of $\mu_{N, t}$ for $N \rightarrow \infty$ and $t \geq 0$ under the condition that the $\mu_{N, 0}$ converge to some probability measure $\mu$. For this we first derive a recurrence equation for the moments of the $\mu_{N, t}$. This will lead to PDEs for the Stieltjes transforms of the $\mu_{N, t}$ and $\mu_{t}$. With the aid of the R-transform from free probability (see Section 5.3 of [AGZ] we then identify the $\mu_{t}$ as free additive convolutions of $\mu$ with suitably scaled semicircle laws. For more details on free probability we refer to [NS.

Denote the $l$-th moment $\left(l \in \mathbb{N}_{0}\right)$ of the probability measure $\mu_{N, t}$ by

$$
S_{N, l}(t):=\int_{\mathbb{R}} y^{l} d \mu_{N, t}(y)=\frac{1}{N^{l / 2+1}}\left(\phi_{N, 1}(t)^{l}+\ldots+\phi_{N, N}(t)^{l}\right) .
$$

Then $S_{N, 0}(t)=1$. Moreover, by (2.1),

$$
\frac{d}{d t} S_{N, 1}(t)=\frac{1}{N^{3 / 2}} \sum_{i, j=1 ; i \neq j}^{N} \frac{1}{\phi_{N, i}(t)-\phi_{N, j}(t)}=0,
$$

i.e., $S_{N, 1}(t)=S_{N, 1}(0)$ for all $t \geq 0$. By the same reasons,

$$
\frac{d}{d t} S_{N, 2}(t)=\frac{2}{N^{2}} \sum_{i, j=1 ; i \neq j}^{N} \frac{\phi_{N, i}(t)}{\phi_{N, i}(t)-\phi_{N, j}(t)}=\frac{2}{N^{2}} \cdot \frac{N(N-1)}{2}=\frac{N-1}{N}
$$

and

$$
\begin{aligned}
\frac{d}{d t} S_{N, 3}(t) & =\frac{3}{N^{5 / 2}} \sum_{i, j=1 ; i \neq j}^{N} \frac{\phi_{N, i}(t)^{2}}{\phi_{N, i}(t)-\phi_{N, j}(t)}=\frac{3}{2 N^{5 / 2}} \sum_{i, j=1 ; i \neq j}^{N}\left(\phi_{N, i}(t)+\phi_{N, j}(t)\right) \\
& =\frac{3(N-1)}{N^{5 / 2}} \sum_{i=1}^{N} \phi_{N, j}(t)=\frac{3(N-1)}{N} S_{N, 1}(0)
\end{aligned}
$$

These computations yield the following result for $l=0,1,2,3$ :

Lemma 2.4. Let $\left(x_{N, k}\right)_{1 \leq k \leq N} \subset \mathbb{R}$ be starting sequences such that for all $l \in \mathbb{N}_{0}$,

$$
c_{l}(0):=\lim _{N \rightarrow \infty} S_{N, l}(0)=\lim _{n \rightarrow \infty} \frac{1}{N^{l / 2+1}}\left(x_{N, 1}^{l}+\ldots+x_{N, N}^{l}\right)<\infty
$$

exists. Then for $l \in \mathbb{N}_{0}$,

$$
c_{l}(t):=\lim _{N \rightarrow \infty} S_{N, l}(t)
$$

exists locally uniformly in $t \in[0, \infty[$ and satisfies the recurrence relation

$$
c_{l}(t)=c_{l}(0)+\frac{l}{2} \int_{0}^{t}\left(\sum_{k=0}^{l-2} c_{l-2-k}(s) c_{k}(s)\right) d s
$$


for $l \geq 2$ and start $c_{0}(t)=1, c_{1}(t)=c_{1}(0)$.

For each $l \in \mathbb{N}_{0}, c_{l}(t)$ is a polynomial in $t$ of degree at most $\lfloor l / 2\rfloor$ with a nonnegative "leading" coefficient of order $\lfloor l / 2\rfloor$.

Proof. By our preceding computations,

$$
c_{0}(t)=1, \quad c_{1}(t)=c_{1}(0), \quad c_{2}(t)=c_{2}(0)+t, \quad c_{3}(t)=c_{3}(0)+3 c_{1}(0) t .
$$

For $l \geq 4$, we have

$$
\begin{aligned}
\frac{d}{d t} S_{N, l}(t) & =\frac{l}{N^{l / 2+1}} \sum_{i, j=1 ; i \neq j}^{N} \frac{\phi_{N, i}(t)^{l-1}}{\phi_{N, i}(t)-\phi_{N, j}(t)} \\
& =\frac{l}{N^{l / 2+1}} \sum_{1 \leq i<j \leq N} \frac{\phi_{N, i}(t)^{l-1}-\phi_{N, j}(t)^{l-1}}{\phi_{N, i}(t)-\phi_{N, j}(t)} \\
& =\frac{l}{2 N^{l / 2+1}} \sum_{i, j=1 ; i \neq j}^{N}\left(\phi_{N, i}(t)^{l-2}+\phi_{N, i}(t)^{l-3} \phi_{N, j}(t)+\ldots+\phi_{N, j}(t)^{l-2}\right) .
\end{aligned}
$$

As

$$
\frac{1}{N^{l / 2+1}} \sum_{i, j=1 ; i \neq j}^{N} \phi_{N, i}(t)^{l-2-k} \phi_{N, j}(t)^{k}=S_{N, l-2-k}(t) S_{N, k}(t)-\frac{S_{N, l-2}(t)}{N}
$$

for $k=1,2, \ldots, l-3$, and as

$$
\frac{1}{N^{l / 2+1}} \sum_{i, j=1 ; i \neq j}^{N} \phi_{N, i}(t)^{l-2}=\frac{N-1}{N} S_{N, l-2}(t),
$$

we get

$$
\begin{aligned}
\frac{d}{d t} S_{N, l}(t) & =\frac{l}{2}\left(\frac{2 N+1-l}{N} S_{N, l-2}(t)+\sum_{k=1}^{l-3} S_{N, l-2-k}(t) S_{N, k}(t)\right) \\
& =\frac{l}{2}\left(\frac{1-l}{N} S_{N, l-2}(t)+\sum_{k=0}^{l-2} S_{N, l-2-k}(t) S_{N, k}(t)\right) .
\end{aligned}
$$

Hence, for $l \geq 4$ we obtain in an inductive way that the limit

$$
\begin{aligned}
c_{l}(t) & :=\lim _{N \rightarrow \infty} S_{N, l}(t)=c_{l}(0)+\lim _{N \rightarrow \infty} \int_{0}^{t} \frac{d}{d s} S_{N, l}(s) d s \\
& =c_{l}(0)+\frac{l}{2} \int_{0}^{t}\left(2 c_{l-2}(s)+\sum_{k=1}^{l-3} c_{l-2-k}(s) c_{k}(s)\right) d s
\end{aligned}
$$

exists locally uniformly in $t \in\left[0, \infty\left[\right.\right.$. Moreover, the $c_{l}(t)$ satisfy

$$
c_{l}(t)=c_{l}(0)+\frac{l}{2} \int_{0}^{t}\left(\sum_{k=0}^{l-2} c_{l-2-k}(s) c_{k}(s)\right) d s .
$$

2.8) and this recurrence imply by an easy induction that for each $l \in \mathbb{N}_{0}, c_{l}(t)$ is a polynomial of degree at most $\lfloor l / 2\rfloor$ with a nonnegative coefficient for this order. 
For even $l$ we next determine the leading coefficients of the polynomials $c_{l}(t)$ of order $l / 2$. For this we recapitulate the Catalan numbers

$$
C_{0}:=1, \quad C_{n}:=\frac{1}{n+1}\left(\begin{array}{c}
2 n \\
n
\end{array}\right)=\left(\begin{array}{c}
2 n \\
n
\end{array}\right)-\left(\begin{array}{c}
2 n \\
n+1
\end{array}\right) \quad(n \geq 1)
$$

which admit the well known recurrence relation (see e.g. Section 2.1.1 of [AGZ]):

$$
C_{0}=C_{1}=1, \quad C_{n+1}=\sum_{k=0}^{n} C_{k} C_{n-k} \quad(n \geq 1) .
$$

We compare this with (2.8) and (2.7) where $c_{l}(t)$ has degree at most $\lfloor l / 2\rfloor$. A simple induction then yields:

Lemma 2.5. The polynomial $c_{2 l}(t)$ has the degree $l$ with the Catalan number $C_{l}$ as leading coefficient for $l \in \mathbb{N}_{0}$.

Example 2.6. Assume that the solutions of our ODEs satisfy $\phi_{N}(0)=0$ for all $N$, i.e., that $x_{N, k}=0$ for all $N, k$. Then $c_{0}(0)=1$ and $c_{l}(0)=0$ for $l \geq 1$. Therefore $c_{0}(t)=1, c_{1}(t)=0, c_{2}(t)=t$ and $c_{3}(t)=0$ for $t \geq 0$. Hence, by (2.7),

$$
c_{2 l}(t)=C_{l} t^{l} \quad \text { and } \quad c_{2 l+1}(t)=0 \quad\left(t \geq 0, l \in \mathbb{N}_{0}\right) .
$$

We next recapitulate that for $R>0$, a random variable $X_{R}$ with the semicircle law $\mu_{s c, R}$ with density $f_{R}(x):=\frac{2}{\pi R^{2}} \sqrt{R^{2}-x^{2}}$ for $|x| \leq R$ and $f_{R}(x)=0$ otherwise has the moments

$$
E\left(X_{R}^{2 n}\right)=\left(\frac{R}{2}\right)^{2 n} C_{n} \quad \text { and } \quad E\left(X_{R}^{2 n+1}\right)=0 \quad \text { for } \quad n \geq 0 ;
$$

see e.g. Section 2.1.1 of AGZ. We thus conclude from the moment convergence theorem that for $t>0$ the empirical measures $\mu_{N, t}$ of the (renormalized) solutions of our ODEs with start in the origin tend weakly to $\mu_{s c, 2 \sqrt{t}}$ for $N \rightarrow \infty$.

If we combine Example 2.6 with Corollary 2.3 for $c=0, t=1 / 2$ there, we obtain the following classical result on the zeroes of the Hermite polynomials; see also [D, G, KM1] for different proofs:

Corollary 2.7. For $N \in \mathbb{N}$ let $z_{1}, \ldots, z_{N}$ be the zeroes of the Hermite polynomial $H_{N}$. Then the normalized empirical measures

$$
\mu_{N}:=\frac{1}{N}\left(\delta_{z_{1} / \sqrt{N}}+\ldots+\delta_{z_{N} / \sqrt{N}}\right)
$$

tend weakly to $\mu_{s c, \sqrt{2}}$ for $N \rightarrow \infty$.

We next study the general case with a start with an arbitrary probability measure $\mu \in M^{1}(\mathbb{R})$ which is determined uniquely by its moments $c_{l}:=\int x^{l} d \mu(x)(l \geq 0)$. This uniqueness holds in particular under the Carleman condition

$$
\sum_{l=1}^{\infty} c_{2 l}^{-\frac{1}{2 l}}=\infty
$$

see p. 85 of A. Moreover, 2.12 clearly follows from the condition

$$
\left|c_{l}\right| \leq(c l)^{l} \quad \text { for all } \quad l \geq 0 \text { and some } \quad c>0 .
$$


Now let $\mu \in M^{1}(\mathbb{R})$ be determined uniquely by its moments $c_{l}$. We choose a family $\left(x_{N, n}\right)_{N \geq 1,1 \leq n \leq N} \subset \mathbb{R}$ of numbers with $x_{N, n-1} \geq x_{N, n}$ for $2 \leq n \leq N$ such that the empirical measures

$$
\mu_{N, 0}:=\frac{1}{N}\left(\delta_{x_{N, 1} / \sqrt{N}}+\ldots \delta_{x_{N, N} / \sqrt{N}}\right)
$$

tend weakly to $\mu$ for $N \rightarrow \infty$, i.e., by the moment convergence theorem, that

$$
\lim _{N \rightarrow \infty} S_{N, l}(0):=\lim _{N \rightarrow \infty} \frac{1}{N^{l / 2+1}}\left(x_{N, 1}^{l}+\ldots+x_{N, N}^{l}\right)=c_{l} \quad(l \geq 0) .
$$

For $N \geq 2$ we now consider the solutions $\phi_{N}(t)$ of 2.1 with start in $\left(x_{N, 1}, \ldots, x_{N, N}\right)$ and the normalized empirical measures

$$
\mu_{N, t}:=\frac{1}{N}\left(\delta_{\phi_{N, 1}(t) / \sqrt{N}}+\ldots+\delta_{\phi_{N, N}(t) / \sqrt{N}}\right) \quad(t \geq 0) .
$$

Proposition 2.8. In the preceding setting, the limits

$$
c_{l}(t):=\lim _{N \rightarrow \infty} S_{N, l}(t) \quad(t \in[0, \infty[, l \geq 0)
$$

exist. Moreover, if the moment condition 2.13) holds for $\mu$, then for each $t \in$ $\left[0, \infty\left[\right.\right.$, the sequence $\left(c_{l}(t)\right)_{l \geq 0}$ is the sequence of moments of some unique probability measure $\mu_{t} \in M^{1}(\mathbb{R})$ for which (2.13) also holds. Moreover, the $\mu_{N, t}$ tend weakly to $\mu_{t}$ for $N \rightarrow \infty$.

Proof. The arguments in the proof of Lemma 2.4 show that the limits $c_{l}(t)$ exist for all $l, t$, and that the $c_{l}(t)$ satisfy the recurrence $(2.7)$.

Assume now that the $c_{l}=c_{l}(0)$ satisfy 2.13 , i.e., $\left|c_{l}\right| \leq(c l)^{l}$ for all $l$ and some $c>0$. We fix $t>0$ and show that there exists $R=R(t)>1$ such that

$$
\left|c_{l}(s)\right| \leq(R l)^{l} \quad \text { for all } \quad l \geq 0, \quad s \in[0, t] .
$$

It is clear from 2.8 that 2.14 holds for $l=0,1,2,3$ and $R$ sufficiently large. Moreover, for $l \geq 4$, we use induction on $l$. In fact, the assumption of our induction, the recurrence (2.7), and the condition (2.13) imply that for $\tau \in[0, t]$,

$$
\begin{aligned}
\left|c_{l}(\tau)\right| & \leq\left|c_{l}(0)\right|+\frac{l}{2} \int_{0}^{\tau}\left(\sum_{k=0}^{l-2}\left|c_{l-2-k}(s)\right| \cdot\left|c_{k}(s)\right|\right) d s \\
& \leq(c l)^{l}+\frac{l(l-1) t}{2} R^{l-2} l^{l-2} .
\end{aligned}
$$

If we choose $R$ large enough depending on $c, t$, we see that the RHS of 2.15 is bounded by $R^{l} l^{l}$, which then proves 2.14 . In summary, for each $t \geq 0$, the sequence $\left(c_{l}(t)\right)_{l}$ satisfies the Carleman condition, and this sequence is the limit of the moment sequences of the measures $\mu_{N, t} \in M^{1}(\mathbb{R})$ for $N \rightarrow \infty$. Hence, by the moment convergence theorem, $\left(c_{l}(t)\right)_{l}$ is the moment sequences of a unique probability measure $\mu_{t} \in M^{1}(\mathbb{R})$, and the $\mu_{N, t}$ tend weakly to $\mu_{t}$.

We next identify the limit measures $\mu_{t}$ in Proposition 2.8 as the free additive convolutions

$$
\mu_{t}=\mu_{s c, 2 \sqrt{t}} \boxplus \mu \quad \text { for } \quad t \geq 0
$$

with the free additive convolution discussed e.g. in [NS, AGZ]. To prove this we need some additional tools. We first recapitulate the Stieltjes transform

$$
G_{\mu}(z):=\int_{\mathbb{R}} \frac{1}{z-x} d \mu(x) \quad \text { for } \quad z \in H:=\{z \in \mathbb{C}: \Im z>0\}
$$


of a probability measure $\mu \in M^{1}(\mathbb{R})$. Clearly, $G_{\mu}$ is analytic on $H$. We next derive PDEs for the Stieltjes transforms

$$
G(t, z):=G_{\mu_{t}}(z), \quad G^{N}(t, z):=G_{\mu_{N, t}}(z) \quad(t \geq 0, z \in H)
$$

of the measures $\mu_{t}$ and $\mu_{N, t}$. In the setting of Proposition 2.8 we now have:

Proposition 2.9. (1) For all $N \in \mathbb{N}, t \geq 0, z \in H$, the partial derivatives of $G^{N}$ satisfy

$$
G_{t}^{N}(t, z)=-G^{N}(t, z) G_{z}^{N}(t, z)-\frac{1}{N} E^{N}(t, z)
$$

with the error term $E^{N}(t, z)$ defined below in 2.19).

(2) Assume that in addition the moment condition 2.13) holds for the start measure $\mu$. Then for $t \geq 0, z \in H$, the function $G$ satisfies Burgers equation

$$
G_{t}(t, z)=-G(t, z) G_{z}(t, z) \text {. }
$$

The appearance of Burgers equation here is not surprising, as this connection is well-known in the context of dynamic versions of Gaussian unitary (or symmetric or symplectic) ensembles; see the next section and e.g. [CG, Men.

Proof. For $t \geq 0$ and $z \in H$ with $|z|$ sufficiently large (depending on $N$ ) we have

$$
G^{N}(t, z)=\int_{\mathbb{R}} \frac{1}{z-x} d \mu_{N, t}(x)=\sum_{l=0}^{\infty} \frac{S_{N, l}(t)}{z^{l+1}}
$$

and thus

$$
G_{t}^{N}(t, z)=\sum_{l=0}^{\infty} \frac{1}{z^{l+1}} \frac{d}{d t} S_{N, l}(t) .
$$

If we apply (2.4)-2.6) as well as the recurrence 2.9), we obtain

$$
\begin{aligned}
G_{t}^{N}(t, z)= & \frac{N-1}{N} \cdot \frac{1}{z^{3}}+\frac{3(N-1)}{N} \cdot S_{N, 1}(0) \cdot \frac{1}{z^{4}} \\
& +\sum_{l=4}^{\infty} \frac{1}{z^{l+1}} \frac{l}{2}\left(\frac{1-l}{N} S_{N, l-2}(t)+\sum_{k=0}^{l-2} S_{N, l-2-k}(t) S_{N, k}(t)\right) \\
= & \sum_{l=2}^{\infty} \frac{1}{z^{l+1}} \frac{l}{2} \sum_{k=0}^{l-2} S_{N, l-2-k}(t) S_{N, k}(t) \quad+\frac{1}{N} E^{N}(t, z)
\end{aligned}
$$

with

$$
E^{N}(t, z):=-\frac{1}{z^{3}}-\frac{3}{z^{4}} \cdot S_{N, 1}(0)+\frac{1}{2} \sum_{l=4}^{\infty} \frac{l(1-l)}{z^{l+1}} \cdot S_{N, l-2}(t) .
$$

Using

$$
z \cdot G_{z}^{N}(t, z)=-\sum_{l=0}^{\infty} \frac{l+1}{z^{l+1}} \cdot S_{N, l}(t), \quad z^{2} \cdot G_{z z}^{N}(t, z)=\sum_{l=0}^{\infty} \frac{(l+1)(l+2)}{z^{l+1}} \cdot S_{N, l}(t),
$$

we obtain by some elementary calculation that

$$
\begin{aligned}
E^{N}(t, z)= & -\frac{1}{2} z^{2} \cdot G_{z z}^{N}(t, z)-2 z \cdot G_{z}^{N}(t, z)-G^{N}(t, z) \\
& -\frac{1}{z^{3}}\left(1-S_{N, 2}(0)-\frac{N-1}{N} t\right)-\frac{3}{z^{4}}\left(S_{N, 1}(0)-\frac{3(N-1)}{N} t-S_{N, 3}(0)\right) .
\end{aligned}
$$


As

$$
\begin{aligned}
& \frac{1}{2 z} \sum_{l=2}^{\infty} \frac{1}{z^{l}} l \sum_{k=0}^{l-2} S_{N, l-2-k}(t) S_{N, k}(t)=\frac{1}{2 z} \sum_{l=0}^{\infty} \sum_{k=0}^{l} \frac{1}{z^{l+2}}(l+2) S_{N, l-k}(t) S_{N, k}(t) \\
& =\frac{1}{2 z^{2}} \sum_{l=0}^{\infty} \sum_{k=0}^{l}\left[(l+1-k) S_{N, l-k}(t) z^{-l-1+k} S_{N, k}(t) z^{-k}\right. \\
& \left.\quad+(k+1) S_{N, l-k}(t) z^{-l+k} S_{N, k}(t) z^{-k-1}\right] \\
& =-G^{N}(t, z) G_{z}^{N}(t, z),
\end{aligned}
$$

part (1) follows for $z \in H$ sufficiently large. As both sides of the equation in (1) are analytic in $z \in H$, this equation holds for all $z \in H$.

For (2) we recapitulate that the measures $\mu_{N, t}$ tend weakly to $\mu_{t}$ by Proposition 2.8. This implies that the Stieltjes transforms $G^{N}(t, z)$ tend to $G(t, z)$ for $t \geq 0$ and locally uniformly for $z \in H$. Hence, by the integral formulas of Cauchy, also $G_{z}^{N}(t, z)$ tends to $G_{z}(t, z)$ and $G_{z z}^{N}(t, z)$ to $G_{z z}(t, z)$ for $z \in H$. Therefore, the error term $E^{N}(t, z)$ converges for $N \rightarrow \infty$ by Lemma 2.4. This and part (1) imply that the derivatives $G_{t}^{N}(t, z)$ tend to $-G(t, z) G_{z}(t, z)$ for $N \rightarrow \infty$. Moreover, as

$$
\left|G_{t}^{N}(t, z)\right|=\left|\frac{d}{d t} \int_{\mathbb{R}} \frac{1}{z-x} d \mu_{N, t}(x)\right| \leq \frac{1}{N} \sum_{k=1}^{N} \frac{1}{\left|z-\phi_{N, k}^{\prime}(t) / \sqrt{N}\right|} \leq \frac{1}{\Im z},
$$

we conclude by dominated convergence that for $t \geq 0$

$$
\lim _{N \rightarrow \infty}\left(G^{N}(t, z)-G^{N}(0, z)\right)=-\int_{0}^{t} G(\tau, z) G_{z}(\tau, z) d \tau .
$$

This implies (2).

Proposition 2.9(2) now leads to 2.16) with the aid of the R-transform of measures $\mu \in M^{1}(\mathbb{R})$ which is defined e.g. in Section 5.3 of AGZ as the formal power series $R_{\mu}(z):=\sum_{n=0}^{\infty} k_{n+1}(\mu) z^{n}$ with the free cumulants $k_{n}(\mu)$ of the measure $\mu$ for which all moments exist. As formal power and Laurent series and also as analytic functions on suitable domains in the upper halfplane (see Section 5.3.3 of [AGZ]), the functions $R_{\mu}$ and $G_{\mu}$ are related by

$$
z-\frac{1}{G_{\mu}(z)}=R_{\mu}\left(G_{\mu}(z)\right)
$$

If we apply this to the measures $\mu_{t}$ and the R-transform $R(t, z):=R_{\mu_{t}}(z)$, we get

$$
z=R(t, G(t, z))+\frac{1}{G(t, z)} .
$$

Hence, on suitable domains,

$$
\begin{aligned}
G_{z}(t, z) & =-\frac{G^{2}(t, z)}{1-R_{z}(t, G(t, z)) G^{2}(t, z)} \\
G_{t}(t, z) & =\frac{R_{t}(t, G(t, z)) G^{2}(t, z)}{1-R_{z}(t, G(t, z)) G^{2}(t, z)} .
\end{aligned}
$$

Proposition $2.9(2)$ now implies that $R_{t}(t, G(t, z))=G(t, z)$. As $z \mapsto G(t, z)$ is not constant, we arrive at

$$
R_{t}(t, z)=z
$$


with $R(0, z)=R_{\mu}(z)$ the R-transform of the starting measure $\mu$. Therefore,

$$
R(t, z)=z t+R(0, z) .
$$

As by 5.3 .23 and 5.3.26 of [AGZ], the R-transform satisfies

$$
R_{\mu_{s c, 2 \sqrt{t}} \boxplus \mu}(z)=R_{\mu_{s c, 2 \sqrt{t}}}(z)+R_{\mu}(z)=z t+R(0, z),
$$

and as the R-transform is injective, we finally obtain (2.16).

In summary, we have proved the following theorem mentioned in the introduction

Theorem 2.10. Let $\mu \in M^{1}(\mathbb{R})$ be a probability measure satisfying 2.13), and let $\left(x_{N, n}\right)_{N \geq 1,1 \leq n \leq N} \subset \mathbb{R}$ with $x_{N, n-1} \geq x_{N, n}$ for $2 \leq n \leq N$ such that the empirical measures

$$
\mu_{N, 0}:=\frac{1}{N}\left(\delta_{x_{N, 1} / \sqrt{N}}+\ldots \delta_{x_{N, N} / \sqrt{N}}\right)
$$

tend weakly to $\mu$ for $N \rightarrow \infty$. If we form the associated solutions $\left(\phi_{N, 1}(t), \ldots, \phi_{N, N}(t)\right)$ of (2.1) and the associated normalized empirical measures

$$
\mu_{N, t}:=\frac{1}{N}\left(\delta_{\phi_{N, 1}(t) / \sqrt{N}}+\ldots+\delta_{\phi_{N, N}(t) / \sqrt{N}}\right) \quad(t \geq 0),
$$

then for $t \in\left[0, \infty\left[\right.\right.$, the $\mu_{N, t}$ tend weakly to $\mu_{s c, 2 \sqrt{t}} \boxplus \mu$.

Remark 2.11. We show in the next section that the limit measures $\mu_{t}(t>0)$ in Proposition 2.8 also appear in a dynamic version Wigner's semicircle law for Gaussian unitary ensembles; c.f. AGZ. If one uses this together with the results of Section 3, one obtains a further proof of 2.16.

We finally study an ODE with an additional drift compared to (2.1). For this let $\phi_{N}\left(t, x_{0}\right)$ be a solution of 2.1 with start in $x_{0} \in C_{N}^{A}$. Then, by an easy computation (see [VW1]),

$$
\tilde{\phi}_{N}\left(t, x_{0}\right):=\phi_{N}\left(\frac{1-e^{-2 \lambda t}}{2 \lambda}, e^{-\lambda t} x_{0}\right)
$$

is a solution of the ODE

$$
\frac{d x}{d t}(t)=H(x(t))-\lambda x(t), \quad \phi_{N}\left(0, x_{0}\right)=x_{0}
$$

for $\lambda \in \mathbb{R}$ and vice versa. As the functions $\tilde{\phi}_{N}$ and $\phi_{N}$ are related by the space-time transformation (2.26), we obtain the following semicircle limit law.

Corollary 2.12. Let $x:=\left(x_{N, k}\right)_{k>1}$ be starting sequences as in Lemma 2.4 and $\lambda>0$. Consider the solutions $\tilde{\phi}_{N}(t):=\tilde{\phi}_{N}\left(t,\left(x_{N, 1}, \ldots, x_{N, 1}\right)\right)$ of 2.27) and the associated normalized empirical measures $\tilde{\mu}_{N, t}$. Then

$$
\lim _{t \rightarrow \infty} \lim _{N \rightarrow \infty} \tilde{\mu}_{N, t}=\lim _{N \rightarrow \infty} \lim _{t \rightarrow \infty} \tilde{\mu}_{N, t}=\mu_{s c, \sqrt{2 / \lambda}} .
$$

Proof. Corollary 2.3 and 2.26 yield $\lim _{t \rightarrow \infty} \tilde{\phi}_{N}(t)=\phi_{N}(1 /(2 \lambda), 0)=\sqrt{1 / \lambda} \cdot z$. Hence, by Example 2.6 and Corollary 2.7, $\lim _{N \rightarrow \infty} \lim _{t \rightarrow \infty} \tilde{\mu}_{N, t}=\mu_{s c, \sqrt{2 / \lambda}}$. 
On the other hand, if we use the moments $\tilde{S}_{N, l}(l \geq 0)$ of the empirical measures $\tilde{\mu}_{N, t}$, we see from the space-time transformation 2.26 and Lemma 2.4 that

$$
\begin{aligned}
\lim _{N \rightarrow \infty} \tilde{S}_{N, l} & =\lim _{N \rightarrow \infty} \frac{1}{N^{l / 2+1}} \sum_{i=1}^{N} \tilde{\phi}_{N, i}(t)^{l} \\
& =\lim _{N \rightarrow \infty} \frac{1}{N^{l / 2+1}} \sum_{i=1}^{N} \phi_{N, i}\left(\frac{1-e^{-2 \lambda t}}{2 \lambda}, e^{-\lambda t}\left(x_{N, 1}, \ldots, x_{N, 1}\right)\right)^{l} \\
& =c_{l}\left(\frac{1-e^{-2 \lambda t}}{2 \lambda}, e^{-\lambda t} x\right),
\end{aligned}
$$

where $c_{l}(t, x)$ is a polynomial in $t$ and $x$ by the proof of Lemma 2.4. Hence

$$
\lim _{t \rightarrow \infty} \lim _{N \rightarrow \infty} \tilde{S}_{N, l}=c_{l}\left(\frac{1}{2 \lambda}, 0\right) .
$$

If we use the recurrence relations for the $c_{l}$ in the proof of Lemma 2.4 together with Example 2.6. we obtain $\lim _{t \rightarrow \infty} \lim _{N \rightarrow \infty} \tilde{\mu}_{N, t}=\mu_{s c, \sqrt{2 / \lambda}}$.

\section{The Semicircle laW for Bessel processes of type A}

Now we consider Bessel processes $\left(X_{t, k}\right)_{t \geq 0}$ on the Weyl chambers $C_{N}^{A}$ for the root systems $A_{N-1}$ which satisfy the SDE

$$
d X_{t, k}^{i}=d B_{t}^{i}+k \sum_{j \neq i} \frac{1}{X_{t, k}^{i}-X_{t, k}^{j}} d t \quad(i=1, \ldots, N) .
$$

with an $N$-dimensional Brownian motion $\left(B_{t}^{1}, \ldots, B_{t}^{N}\right)_{t \geq 0}$. By GrM] (see also Sch for a related situation) we know that for $k \geq 1 / 2$ and all starting points $x \in C_{N}^{A}$, (3.1) admits an a.s. solution $\left(X_{t, k}\right)_{t \geq 0}$ which does not hit the boundary of $C_{N}^{A}$ for $t>0$ almost surely, even if $x$ is on the boundary of $C_{N}^{A}$. In the following we only consider this regular case $k \geq 1 / 2$.

Under convergence conditions on the starting points as in Section 2 for $N \geq 2$, we now derive limit theorems for the moments of the associated empirical measures

$$
\mu_{N, t}:=\frac{1}{N}\left(\delta_{X_{t, k}^{1} / \sqrt{N}}+\ldots+\delta_{X_{t, k}^{N} / \sqrt{N}}\right)
$$

for $t \geq 0$ and $N \rightarrow \infty$. For this, it will be convenient also to study the renormalized processes $\left(\tilde{X}_{t, k}:=X_{t, k} / \sqrt{k}\right)_{t \geq 0}$ which satisfy the SDE

$$
d \tilde{X}_{t, k}^{i}=\frac{1}{\sqrt{k}} d B_{t}^{i}+\sum_{j \neq i} \frac{1}{\tilde{X}_{t, k}^{i}-\tilde{X}_{t, k}^{j}} d t \quad(i=1, \ldots, N),
$$

which agrees, for $k=\infty$, with the ODE 2.1). We also study the empirical measures

$$
\tilde{\mu}_{N, t}:=\frac{1}{N}\left(\delta_{\tilde{X}_{t, k}^{1} / \sqrt{N}}+\ldots+\delta_{\tilde{X}_{t, k}^{N} / \sqrt{N}}\right) .
$$

Denote the $l$-th moment $\left(l \in \mathbb{N}_{0}\right)$ of $\tilde{\mu}_{N, t}$ by

$$
S_{N, l}(t):=\int_{\mathbb{R}} y^{l} d \tilde{\mu}_{N, t}(y)=\frac{1}{N^{l / 2+1}} \sum_{i=1}^{N}\left(\tilde{X}_{t, k}^{i}\right)^{l} .
$$

We will show that for all $l$ the moments $S_{N, l}(t)$ converge for $N \rightarrow \infty$ to the numbers $c_{l}(t)$ of Lemma 2.4 independent of $k \in[1 / 2, \infty]$. The proof of this fact will 
be based on some induction which even leads to a slightly more general convergence statement.

To state this result, we need some notation about partitions. Let $\mathcal{P}$ the set of all partitions with $N$ components consisting of all $\lambda=\left(\lambda_{1}, \ldots, \lambda_{N}\right) \in \mathbb{N}_{0}^{N}$ with $\lambda_{1} \geq \lambda_{2} \geq \ldots \geq \lambda_{N}$. For $\lambda \in \mathcal{P}$, let $|\lambda|=\lambda_{1}+\ldots+\lambda_{N}$ its weight and $L(\lambda):=$ $\max \left\{k: \lambda_{k} \geq 1\right\}$ its length. We also consider the symmetric monomials

$$
m_{\lambda}(x):=\sum_{\pi \in S_{N}} x^{\pi(\lambda)}:=\sum_{\pi \in S_{N}} x_{1}^{\lambda_{\pi(1)}} x_{2}^{\lambda_{\pi(2)}} \cdots x_{N}^{\lambda_{\pi(N)}}
$$

for $x=\left(x_{1}, \ldots, x_{N}\right) \in \mathbb{R}^{N}$ where the sum runs over the symmetric group $S_{N}$ which acts on vectors in the obvious way.

Lemma 3.1. Let $\left(x_{N}\right)_{N \geq 1}:=\left(x_{N, n}\right)_{N \geq 1,1 \leq n \leq N} \subset \mathbb{R}$ be a family of starting numbers with $x_{N, n-1} \geq x_{N, n}$ for $2 \leq n \leq N$, for which

$$
\lim _{N \rightarrow \infty} \frac{m_{\lambda}\left(x_{N}\right)}{N ! \cdot N^{|\lambda| / 2}}<\infty
$$

exists for all $\lambda \in \mathcal{P}$. Let $k \in[1 / 2, \infty]$, and consider for $N \geq 2$ the renormalized Bessel processes $\left(\tilde{X}_{t, k}\right)_{t \geq 0}$ with start in $\left(x_{N, 1}, \ldots, x_{N, n}\right) \in C_{N}^{A}$. Then, for all $\lambda \in \mathcal{P}$, the limits

$$
\lim _{N \rightarrow \infty} \frac{E\left(m_{\lambda}\left(\tilde{X}_{t, k}\right)\right)}{N ! \cdot N^{|\lambda| / 2}}
$$

exist locally uniformly in $t$ and are independent from $k$.

Proof. We prove this statement by induction on $|\lambda|$.

For $|\lambda|=0$ we have $\lambda=0$ and $m_{\lambda}(x)=N$ ! which yields the claim here. For $|\lambda|=1$ we have $\lambda=(1,0, \ldots, 0)$ and $m_{\lambda}(x)=(N-1) ! \cdot\left(x_{1}+\ldots+x_{N}\right)$. Thus, as

$$
\sum_{i=1}^{N} \tilde{X}_{t, k}^{i}=\sum_{i=1}^{N} x_{N, i}+\frac{1}{\sqrt{k}} \sum_{i=1}^{N} B_{t}^{i}
$$

by $(3.3)$, the claim also follows.

Now let $\lambda \in \mathcal{P}$ with $|\lambda| \geq 2$, and we assume that the statement is already shown for partitions with weight at most $|\lambda|-1$. Itô's formula and 3.3 yield

$$
\begin{aligned}
m_{\lambda}\left(\tilde{X}_{t, k}\right)= & m_{\lambda}\left(x_{N}\right)+\frac{1}{\sqrt{k}} \sum_{i=1}^{N} \int_{0}^{t} \frac{d m_{\lambda}}{d x_{i}}\left(\tilde{X}_{s, k}\right) d B_{s}^{i} \\
& +\int_{0}^{t}\left(\sum_{i=1}^{N} \frac{1}{2 k} \frac{d^{2} m_{\lambda}}{d x_{i}^{2}}\left(\tilde{X}_{s, k}\right)+\sum_{i=1}^{N} \sum_{j \neq i} \frac{d m_{\lambda}}{d x_{i}}\left(\tilde{X}_{s, k}\right) \cdot \frac{1}{\tilde{X}_{s, k}^{i}-\tilde{X}_{s, k}^{j}}\right) d s .
\end{aligned}
$$

We now claim that the diffusion parts $\frac{1}{\sqrt{k}} \sum_{i=1}^{N} \int_{0}^{t} \frac{d m_{\lambda}}{d x_{i}}\left(\tilde{X}_{s, k}\right) d B_{s}^{i}$ of 3.5 are martingales and thus

$$
E\left(\sum_{i=1}^{N} \int_{0}^{t} \frac{d m_{\lambda}}{d x_{i}}\left(\tilde{X}_{s, k}\right) d B_{s}^{i}\right)=0 \quad(t \geq 0) .
$$

To prove this we observe that

$$
\sum_{i=1}^{N} \int_{0}^{t} \frac{d m_{\lambda}}{d x_{i}}\left(\tilde{X}_{s, k}\right) d B_{s}^{i} \stackrel{d}{=} \int_{0}^{t} \sqrt{\sum_{i=1}^{N}\left(\frac{d m_{\lambda}}{d x_{i}}\left(\tilde{X}_{s, k}\right)\right)^{2}} d \tilde{B}_{s}
$$


for some one-dimensional Brownian motion $\tilde{B}$ by the Lévy characterization of the one-dimensional Brownian motion. We now analyze the integrand of the RHS of (3.7). As $\sum_{i=1}^{N}\left(\frac{d m_{\lambda}}{d x_{i}}\right)^{2}$ is a symmetric polynomial, we may write it as a polynomial expression with rational coefficients of polynomials of the form $p_{n}(x)=\sum_{i=1}^{N} x_{i}^{n}$. Furthermore, for $l \geq 1$, we have

$$
\sum_{i=1}^{N} x_{i}^{2 l} \leq\left(\sum_{i=1}^{N} x_{i}^{2}\right)^{l}, \quad \sum_{i=1}^{N}\left|x_{i}\right|^{2 l+1} \leq\left(\sum_{i=1}^{N} x_{i}^{2}\right)^{(2 l+1) / 2} .
$$

As $\sum_{i=1}^{N} \tilde{X}_{s, k}^{i}=\sum_{i=1}^{N} B_{s}^{i}$, and as $\left(\sum_{i=1}^{N}\left(\tilde{X}_{s, k}^{i}\right)^{2}\right)_{s \geq 0}$ is a classical squared Bessel process by

$$
\sum_{i=1}^{N}\left(\tilde{X}_{t, k}^{i}\right)^{2}=\sum_{i=1}^{N} x_{N, i}^{2}+\frac{2}{\sqrt{k}} \sum_{i=1}^{N} \int_{0}^{t} \tilde{X}_{s, k}^{i} d B_{s}^{i}+\left(N(N-1)+\frac{N}{k}\right) t,
$$

we see that we may bound $\sum_{i=1}^{N}\left(\frac{d m_{\lambda}}{d x_{i}}\left(\tilde{X}_{s, k}\right)\right)^{2}$ by a polynomial in a one-dimensional Brownian motion and a one-dimensional squared Bessel process. With standard results on the Itô integral, this readily yields the claim and 3.6 .

We now turn to the second term in the RHS of 3.5 . We here use that

$$
\sum_{i=1}^{N} \frac{d^{2} m_{\lambda}}{d x_{i}^{2}}(x)=\sum_{i=1}^{N} \sum_{\pi \in S_{N}} \lambda_{\pi(i)}\left(\lambda_{\pi(i)}-1\right) x^{\pi(\lambda)-2 e_{i}}
$$

with the $i$-th unit vector $e_{i}=(0, \ldots, 0,1,0, \ldots, 0) \in \mathbb{R}^{N}$. Notice that this expression is a symmetric polynomial which is homogeneous of order $|\lambda|-2$ and thus a linear combination of the symmetric monomials $m_{\tilde{\lambda}}$ with $\tilde{\lambda} \in \mathcal{P}$ with $|\tilde{\lambda}|=|\lambda|-2$. Moreover, the coefficients in this linear combination depend only on $\lambda_{1}, \ldots, \lambda_{L(\lambda)}$ and not on $N \geq L(\lambda)$. Hence, by our induction assumption,

$$
\frac{1}{N ! \cdot N^{|\lambda| / 2}} E\left(\int_{0}^{t} \sum_{i=1}^{N} \frac{1}{2 k} \frac{d^{2} m_{\lambda}}{d x_{i}^{2}}\left(\tilde{X}_{s, k}\right) d s\right)=O(1 / N) .
$$

We now turn to the $3 \mathrm{rd}$ term in the RHS of (3.5). We here first notice that for $u, v \in \mathbb{R}, a, b \in \mathbb{N}_{0},\left(u^{a} v^{b}-u^{b} v^{a}\right) /(u-v)$ is some polynomial $p_{a, b}(u, v)$ of degree $a+b-1$. With our notations and the transposition $(i, j) \in S_{N}$ we here have

$$
\begin{aligned}
\sum_{i=1}^{N} \sum_{j \neq i} & \frac{d m_{\lambda}}{d x_{i}}(x) \cdot \frac{1}{x_{i}-x_{j}}=\sum_{\pi \in S_{N}} \sum_{i=1}^{N} \sum_{j \neq i} \lambda_{\pi(i)} \frac{x^{\pi(\lambda)-e_{i}}}{x_{i}-x_{j}} \\
= & \frac{1}{2} \sum_{\pi \in S_{N}} \sum_{i=1}^{N} \sum_{j \neq i} \frac{\lambda_{\pi(i)} x^{\pi(\lambda)-e_{i}}-\lambda_{\pi(j)} x^{\pi(\lambda)-e_{j}}}{x_{i}-x_{j}} \\
= & \frac{1}{2} \sum_{\pi \in S_{N}} \sum_{i=1}^{N} \sum_{j \neq i} \frac{\lambda_{\pi(i)} x^{\pi(\lambda)-e_{i}}-\lambda_{\pi(i)} x^{\pi \circ(i, j)(\lambda)-e_{j}}}{x_{i}-x_{j}} \\
= & \frac{1}{2} \sum_{\pi \in S_{N}} \sum_{i=1}^{N} \sum_{j \neq i} \lambda_{\pi(i)} p_{\lambda_{\pi(i)}-1, \lambda_{\pi(j)}-1}\left(x_{i}, x_{j}\right) \cdot\left(x^{\pi(\lambda)}\right)_{i, j}
\end{aligned}
$$

where $\left(x^{\pi(\lambda)}\right)_{i, j}$ means the product as in the definition of the $m_{\lambda}$ above where factors involving $x_{i}, x_{j}$ are cancelled, as these parts are handled separately in the 
polynomial $p_{\lambda_{\pi(i)}-1, \lambda_{\pi(j)}-1}\left(x_{i}, x_{j}\right)$. Please notice that the third $=$ in 3.9 follows by an obvious substitution in the summation over $\pi \in S_{N}$ in the second summand.

The expression (3.9) obviously is a symmetric polynomial which is homogeneous of order $|\lambda|-2$ and thus a linear combination of the symmetric monomials $m_{\tilde{\lambda}}$ with $\tilde{\lambda} \in \mathcal{P}$ with $|\tilde{\lambda}|=|\lambda|-2$. Moreover, if we analyze the coefficients $c_{\tilde{\lambda}}$ of the $m_{\tilde{\lambda}}$ of this linear combination (w.l.o.g. take for simplicity the product $x^{\tilde{\lambda}}$ ), we see that $c_{\tilde{\lambda}} / N$ converges for $N \rightarrow \infty$. Hence, by our induction assumption,

$$
\frac{1}{N ! \cdot N^{|\lambda| / 2}} E\left(\int_{0}^{t} \sum_{i=1}^{N} \sum_{j \neq i} \frac{d m_{\lambda}}{d x_{i}}\left(\tilde{X}_{s, k}\right) \cdot \frac{1}{\tilde{X}_{s, k}^{i}-\tilde{X}_{s, k}^{j}}\right) d s .
$$

converges for $N \rightarrow \infty$. A combination of (3.5), (3.8), 3.9), and 3.10 now completes our induction.

Remark 3.2. The proof of Lemma 3.1 shows that for fixed $k$ and $\lambda$, the limit in Lemma 3.1 has order $O(1 / N)$.

Lemma 3.1 has the following application to the moments $S_{N, l}(t)$ :

Corollary 3.3. Let $\left(x_{N, n}\right)_{N \geq 1,1 \leq n \leq N} \subset \mathbb{R}$ be starting numbers with $x_{N, n-1} \geq x_{N, n}$ for $2 \leq n \leq N$, for which the convergence condition in Lemma 2.4 holds. Let $k \in[1 / 2, \infty]$, and let for $N \geq 2,\left(\tilde{X}_{t, k}\right)_{t \geq 0}$ the renormalized Bessel processes starting in $\left(x_{N, 1}, \ldots, x_{N, n}\right) \in C_{N}^{A}$. Then, for $l \in \mathbb{N}_{0}$ and $c_{l}(t)$ from Lemma 2.4.

$$
E\left(S_{N, l}(t)\right) \rightarrow c_{l}(t) \quad \text { for } \quad N \rightarrow \infty
$$

Proof. As the polynomials $m_{\lambda}$ in Lemma 3.1 are polynomials in the polynomials $\sum_{i=1}^{N} x_{i}^{l}$ for $l \leq|\lambda|$ with coefficient independent of $N$ for $N \geq L(\lambda)$, the convergence condition in Lemma 2.4 implies that the convergence condition in Lemma 3.1 holds. As $S_{N, l}(t)=\frac{1}{N ! N^{l / 2}} m_{(l, 0, \ldots, 0)}\left(\tilde{X}_{t, k}\right)$, Lemma 3.1 implies that the expectations converge, and that the limit is independent from $k \in[1 / 2, \infty]$. Lemma 2.4 now completes the proof.

Corollary 3.3 can be extended to an a.s. result:

Theorem 3.4. Let $\left(x_{N, n}\right)_{N \geq 1,1 \leq n \leq N} \subset \mathbb{R}$ be starting numbers with $x_{N, n-1} \geq x_{N, n}$ for $2 \leq n \leq N$, for which the condition in Lemma 2.4 holds. Fix $k \geq 1 / 2$, and, for $N \geq 2$, the renormalized Bessel processes $\left(\tilde{X}_{t, k}\right)_{t \geq 0}$ starting in $\left(x_{N, 1}, \ldots, x_{N, n}\right) \in$ $C_{N}^{A}$. Then,

$$
S_{N, l}(t) \rightarrow c_{l}(t) \quad \text { for } \quad N \rightarrow \infty
$$

almost surely for all $l \geq 0$ and locally uniformly for $t \in[0, \infty[$.

Proof. We first recapitulate from Corollary 3.3 that for all $l$,

$$
E\left(\frac{1}{N^{l / 2+1}} \sum_{i=1}^{N}\left(\tilde{X}_{t, k}^{i}\right)^{l}\right)=c_{l}(t)+o(1) \quad(N \rightarrow \infty)
$$

locally uniformly in $t \geq 0$. Moreover, by Itô's formula and (3.3),

$$
\begin{aligned}
\sum_{i=1}^{N}\left(\tilde{X}_{t, k}^{i}\right)^{l}= & \sum_{i=1}^{N} x_{N, i}^{l}+\frac{l}{\sqrt{k}} \sum_{i=1}^{N} \int_{0}^{t}\left(\tilde{X}_{s, k}^{i}\right)^{l-1} d B_{s}^{i} \\
& +\int_{0}^{t}\left(l \sum_{i=1}^{N} \sum_{j \neq i} \frac{\left(\tilde{X}_{s, k}^{i}\right)^{l-1}}{\tilde{X}_{s, k}^{i}-\tilde{X}_{s, k}^{j}}+\sum_{i=1}^{N} \frac{l(l-1)}{2 k}\left(\tilde{X}_{s, k}^{i}\right)^{l-2}\right) d s .
\end{aligned}
$$


This together with the Chebychev and Burkholder-Davis-Gundy inequality shows that for all $l \in \mathbb{N}_{0}, \epsilon>0$ and $T>0$ and some universal constant $c>0$,

$$
\begin{aligned}
\sup _{s \leq T} P( & \mid \frac{1}{N^{l / 2+1}}\left(\sum _ { i = 1 } ^ { N } \left(\left(\tilde{X}_{s, k}^{i}\right)^{l}-\sum_{i=1}^{N} x_{N, i}^{l}\right.\right. \\
& \left.\left.-\int_{0}^{t}\left(l \sum_{i=1}^{N} \sum_{j \neq i} \frac{\left(\tilde{X}_{s, k}^{i}\right)^{l-1}}{\tilde{X}_{s, k}^{i}-\tilde{X}_{s, k}^{j}}+\sum_{i=1}^{N} \frac{l(l-1)}{2 k}\left(\tilde{X}_{s, k}^{i}\right)^{l-2}\right) d s\right) \mid>\epsilon\right) \\
& \leq \frac{1}{\epsilon^{2}} E\left(\sup _{s \leq T}\left(\frac{1}{N^{l / 2+1}} \frac{l}{\sqrt{k}} \sum_{i=1}^{N} \int_{0}^{t}\left(\tilde{X}_{s, k}^{i}\right)^{l-1} d B_{s}^{i}\right)^{2}\right) \\
& \leq \frac{c}{\epsilon^{2}} E\left(\frac{l^{2}}{k} \frac{1}{N^{l+2}} \int_{0}^{T} \sum_{i=1}^{N}\left(\tilde{X}_{s, k}^{i}\right)^{2 l-2} d s\right) \rightarrow 0
\end{aligned}
$$

as $N \rightarrow \infty$. Furthermore, by 3.12 ,

$$
\sum_{N=1}^{\infty} E\left(\frac{1}{N^{2}} \frac{l^{2}}{k} \int_{0}^{T} \frac{1}{N^{l}} \sum_{i=1}^{N}\left(X_{s, k}^{i}\right)^{2 l-2} d s\right)<\infty .
$$

This and the Borel-Cantelli Lemma imply that

$$
\begin{aligned}
\frac{1}{N^{l / 2+1}} & \left(\sum_{i=1}^{N}\left(\tilde{X}_{s, k}^{i}\right)^{l}-\sum_{i=1}^{N} x_{N, i}^{l}\right. \\
& \left.-\int_{0}^{t}\left(l \sum_{i=1}^{N} \sum_{j \neq i} \frac{\left(\tilde{X}_{s, k}^{i}\right)^{l-1}}{\tilde{X}_{s, k}^{i}-\tilde{X}_{s, k}^{j}}+\sum_{i=1}^{N} \frac{l(l-1)}{2 k}\left(\tilde{X}_{s, k}^{i}\right)^{l-2}\right) d s\right) \rightarrow 0
\end{aligned}
$$

for $N \rightarrow \infty$ almost surely. Hence, by (3.13), also

$$
\frac{1}{N^{l / 2+1}}\left(\sum_{i=1}^{N} \frac{l}{\sqrt{k}} \int_{0}^{t}\left(\tilde{X}_{s, k}^{i}\right)^{l-1} d B_{s}^{i}\right) \rightarrow 0 \quad \text { a.s. }
$$

With this result we now prove the claim by induction on $l$. In fact, the case $l=0$ is trivial, and the case $l=1$ follows easily from $(3.13)$ and $(3.14)$ for $l=1$, and the fact that the drift part on the RHS of (3.13) disappears. Moreover, for $l \geq 2$, we again use (3.13) and (3.14), i.e., it suffices to show that for $N \rightarrow \infty$

$$
\begin{aligned}
& \frac{1}{N^{l / 2+1}}\left(\sum_{i=1}^{N} x_{N, i}^{l}+\int_{0}^{t}\left(l \sum_{i=1}^{N} \sum_{j \neq i} \frac{\left(\tilde{X}_{s, k}^{i}\right)^{l-1}}{\tilde{X}_{s, k}^{i}-\tilde{X}_{s, k}^{j}}+\sum_{i=1}^{N} \frac{l(l-1)}{2 k}\left(\tilde{X}_{s, k}^{i}\right)^{l-2}\right) d s\right) \\
& \quad=c_{l}(t)+o_{p}\left(\frac{1}{N^{l / 2+1}}\right)
\end{aligned}
$$

almost surely. But this follows easily from our induction assumption and the recurrence of the numbers $c_{l}(t)$ in Lemma 2.4. This yields the claim.

Theorem 3.4 implies that for $N \rightarrow \infty$ the moments of the empirical measures of the renormalized Bessel processes $\left(\tilde{X}_{t, k}\right)_{t \geq 0}$ behave almost surely like the solutions of the ODEs 2.1], i.e. the case $k=\infty$. Hence Theorem 2.10 also holds for the empirical measure associated to the renormalized Bessel processes $\left(\tilde{X}_{t, k}\right)_{t \geq 0}$. In particular, we obtain the following result from the moment convergence theorem. 
Theorem 3.5. Let $\mu \in M^{1}(\mathbb{R})$ be a probability measure satisfying 2.13), and let $\left(x_{N, n}\right)_{N \geq 1,1 \leq n \leq N} \subset \mathbb{R}$ with $x_{N, n-1} \geq x_{N, n}$ for $2 \leq n \leq N$ such that the associated normalized empirical measures as in (2.25) tend weakly to $\mu$ for $N \rightarrow \infty$.

For $k \geq 1 / 2$ and $N \in \mathbb{N}$, consider the renormalized Bessel processes $\left(\tilde{X}_{t, k}\right)_{t \geq 0}$ with start in $\left(x_{N, 1}, \ldots, x_{N, n}\right) \in C_{N}^{A}$. Then, for $t \geq 0$, the empirical measures $\tilde{\mu}_{N, t}$ from (3.4) tend weakly to $\mu_{s c, 2 \sqrt{t}} \boxplus \mu$ almost surely for $N \rightarrow \infty$.

Remark 3.6. The previous results were obtained for fixed $k>1 / 2$. As in AV1, AV2, V], VW1 we may also consider the freezing regime $k \rightarrow \infty$. Here we choose starting points $\left(x_{1, N}, \ldots, x_{N, N}\right)$ in the interior of $C_{N}^{A}$ for $N \in \mathbb{N}$. Then by [AV1,

$$
X_{t, k} / \sqrt{k} \rightarrow \phi_{N}(t)
$$

locally uniform in $t$ a.s. for $k \rightarrow \infty$ and the Bessel processes $\left(X_{t, k}^{N}\right)_{t \geq 0}$ starting in $\left(x_{1, N}, \ldots, x_{N, N}\right)$ where the $\phi_{N}$ are the solutions of $(2.1)$ with start in $\left(x_{1, N}, \ldots, x_{N, N}\right)$. Hence, by (2.4),

$$
\lim _{N \rightarrow \infty} \lim _{k \rightarrow \infty} \frac{1}{N^{l / 2+1}} \sum_{i=1}^{N}\left(\frac{X_{t, k}^{i}}{\sqrt{k}}\right)^{l}=c_{l}(t)
$$

a.s.. On the other hand, by Theorem 3.4 ,

$$
\lim _{k \rightarrow \infty} \lim _{N \rightarrow \infty} \frac{1}{N^{l / 2+1}} \sum_{i=1}^{N}\left(\frac{X_{t, k}^{i}}{\sqrt{k}}\right)^{l}=c_{l}(t)
$$

a.s., i.e., the limits of $k$ and $N$ may be interchanged here.

We next study a stochastic analogue of Corollary 2.12 and modify the SDE of the Bessel processes by an additional drift $-\lambda x$ with some constant $\lambda \in \mathbb{R}$, i.e. a component as in a classical Ornstein-Uhlenbeck setting, cf. VWW. We thus consider Bessel-OU processes $Y:=\left(Y_{t, k}\right)_{t \geq 0}$ on $C_{N}^{A}$ of type $A_{N-1}$ as solutions of

$$
d Y_{t, k}^{i}=d B_{t}^{i}+\left(k \sum_{j \neq i} \frac{1}{Y_{t, k}^{i}-Y_{t, k}^{j}}-\lambda Y_{t, k}^{i}\right) d t \quad(i=1, \ldots, N)
$$

for $k \geq 1 / 2$ with an $N$-dimensional Brownian motion $\left(B_{t}^{1}, \ldots, B_{t}^{N}\right)_{t \geq 0}$. For $\lambda>0$, these processes are mean reverting ergodic process $Y=\left(Y_{t, k}\right)_{t \geq 0}$ with speed of mean-reversion $\lambda$, and for $\lambda \leq 0$, non-ergodic.

Itô's formula together with a time-change argument shows that $Y$ is a space-time transformation of the original Bessel process $\left(X_{t, k}\right)_{t \geq 0}($ with $\lambda=0)$ via

$$
Y_{t, k}=e^{-\lambda t} X_{\frac{e^{2 \lambda t}-1}{2 \lambda}, k} \text {. }
$$

For a proof based on the generators of these diffusions see RV1]. Using this spacetime transformation, we can immediately reformulate Theorems 3.4 and 3.5 for the $Y_{t, k}$. Moreover, for $t \rightarrow \infty$ we obtain the following analogue to Corollary 2.12,

Corollary 3.7. Consider starting sequences $\left(x_{1, N}, \ldots, x_{N, N}\right) \subset C_{N}^{A}$ for $N \in \mathbb{N}$ as in Lemma 2.4. and let $\lambda>0$ and $k \geq 1 / 2$. For $N \in \mathbb{N}$ let $\left(Y_{t, k}^{N}\right)_{t \geq 0}$ be the solution of (3.15) on $C_{N}^{A}$ with start in $\left(x_{1, N}, \ldots, x_{N, N}\right)$. Then the empirical measures

$$
\tilde{\mu}_{N, t}:=\frac{1}{N}\left(\delta_{Y_{t, k}^{1} / \sqrt{N}}+\ldots+\delta_{Y_{t, k}^{N} / \sqrt{N}}\right)
$$

satisfy $\lim _{N \rightarrow \infty} \lim _{t \rightarrow \infty} \tilde{\mu}_{N, t}=\lim _{t \rightarrow \infty} \lim _{N \rightarrow \infty} \tilde{\mu}_{N, t}=\mu_{s c, \sqrt{2 / \lambda}}$. 


\section{Zeroes of Laguerre polynomials and the Marchenko-Pastur law}

In this section we transfer the approach of Section 2 for the empirical measures of the zeroes of the Hermite polynomials to Laguerre polynomials. We start with the appropriate ODE:

The ODE 4.1. Let $\nu \in] 0, \infty[$ and $N \geq 2$. On the interior of the closed Weyl chamber

$$
C_{N}^{B}:=\left\{x \in \mathbb{R}^{N}: \quad x_{1} \geq x_{2} \geq \ldots \geq x_{N} \geq 0\right\} \subset \mathbb{R}^{N}
$$

of type $\mathrm{B}$, we consider the $\mathbb{R}^{N}$-valued function

$$
H_{\nu}(x):=\left(\sum_{j \neq 1} \frac{2 x_{1}}{x_{1}^{2}-x_{j}^{2}}+\frac{\nu}{x_{1}}, \ldots, \sum_{j \neq N} \frac{2 x_{N}}{x_{N}^{2}-x_{j}^{2}}+\frac{\nu}{x_{N}}\right) .
$$

It is shown in [VW2 that for each initial condition $x_{0} \in C_{N}^{B}$, the ODE

$$
\frac{d x}{d t}(t)=H_{\nu}(x(t)), \quad x(0)=x_{0}
$$

has a unique solution for all $t \geq 0$ in the sense that $\left[0, \infty\left[\rightarrow C_{N}^{B}, t \mapsto x(t)\right.\right.$ is continuous where $x(t)$ is in the interior of $C_{N}^{B}$ and solves the ODE in 4.1 for $t>0$. For the ODE 4.1 see also AV1, VW1. We denote solutions of the ODE (2.1) by $\phi_{N}:=\left(\phi_{N, 1}, \ldots, \phi_{N, N}\right)$ where we suppress the dependence on $x_{0}$ and $\nu$.

For $x_{0}=0 \in C_{N}^{b}$, the solution of 4.1 can be expressed in terms of the zeroes of the Laguerre polynomial $L_{N}^{(\nu-1)}$ where the $\left(L_{N}^{(\nu-1)}\right)_{N \geq 0}$ are orthogonal w.r.t. the density $e^{-x} x^{\nu-1}$ on $] 0, \infty[$ as in $[\mathrm{Sz}]$. In fact, by [AV1] we have:

Lemma 4.2. Let $\nu>0$ and $z_{1}^{(\nu-1)}>\ldots>z_{N}^{(\nu-1)}>0$ be the ordered zeros of $L_{N}^{(\nu-1)}$. Then, for $z:=\left(z_{1}^{(\nu-1)}, \ldots, z_{N}^{(\nu-1)}\right) \in C_{N}^{B}$ and any $c \geq 0$,

$$
\phi_{N}(t):=\sqrt{2 t+c^{2}} \cdot\left(\sqrt{z_{1}^{(\nu-1)}}, \ldots, \sqrt{z_{N}^{(\nu-1)}}\right) \quad(t \geq 0)
$$

is a solution of the ODE (4.1).

We now study the empirical measures of solutions of 4.1. We proceed as in Section 2 and derive recurrence relations for the associated empirical moments. However, this works for the even moments only. For this reason we take squares in all components of $\phi_{N}$ at some stages. As we are working here on the Weyl chambers $C_{N}^{B}$, no information is lost by taking these squares. Moreover, having the well-known relation $H_{2 N}(x)=d_{N} L_{N}^{(-1 / 2)}\left(x^{2}\right)\left(d_{N}>0\right.$ some constants) in mind (see (5.6.1) of $[\mathrm{Sz}]$ ), we normalize our empirical measures with $2 N$ instead of $N$.

We now choose a family $\left(x_{N, k}\right)_{1 \leq k \leq N} \in\left[0, \infty\left[\right.\right.$ with $\left(x_{N, 1}, \ldots, x_{N, N}\right) \in C_{N}^{B}$ for $N \geq 2$. We then form the associated solutions $\phi_{N}(t)$ of (4.1) with start in $\left(x_{N, 1}, \ldots, x_{N, N}\right)$ for $N \geq 2$, and introduce the normalized empirical measures

$$
\mu_{N, t}^{2}:=\frac{1}{N}\left(\delta_{\frac{\phi_{N, 1}(t)^{2}}{2 N}}+\ldots+\delta_{\frac{\phi_{N, N}(t)^{2}}{2 N}}\right)
$$

for $t \geq 0$. Denote the $l$-th moment $\left(l \in \mathbb{N}_{0}\right)$ of $\mu_{N, t}^{2}$ by

$$
S_{N, l}(t):=\int_{\mathbb{R}} y^{l} \mu_{N, t}(y)=\frac{1}{2^{l} N^{l+1}}\left(\phi_{N, 1}(t)^{2 l}+\ldots+\phi_{N, N}(t)^{2 l}\right) .
$$


Then $S_{N, 0}(t)=1$. Moreover, by (4.1),

$$
\frac{d}{d t} S_{N, 1}(t)=\frac{2}{2 N^{2}}\left(\sum_{i, j=1 ; i \neq j}^{N} \frac{2 \phi_{N, i}(t)^{2}}{\phi_{N, i}(t)^{2}-\phi_{N, j}(t)^{2}}+\nu N\right)=\frac{N(N+\nu-1)}{N^{2}} .
$$

Furthermore, for $l \geq 2$,

$$
\begin{aligned}
& \frac{d}{d t} S_{N, l}(t)=\frac{2}{2^{l} N^{l+1}}\left(\sum_{i, j=1 ; i \neq j}^{N} \frac{2 \phi_{N, i}(t)^{2 l}}{\phi_{N, i}(t)^{2}-\phi_{N, j}(t)^{2}}+\nu \sum_{i=1}^{N} \phi_{N, 1}(t)^{2 l-2}\right) \\
& =\frac{2}{2^{l} N^{l+1}}\left(2 \sum_{1 \leq i<j \leq N} \frac{\phi_{N, i}(t)^{2 l}-\phi_{N, j}(t)^{2 l}}{\phi_{N, i}(t)^{2}-\phi_{N, j}(t)^{2}}+\nu \sum_{i=1}^{N} \phi_{N, 1}(t)^{2 l-2}\right) \\
& =\frac{2}{2^{l} N^{l+1}}\left(\sum_{i, j=1 ; i \neq j}^{N}\left(\phi_{N, i}(t)^{2(l-1)}+\phi_{N, i}(t)^{2(l-2)} \phi_{N, j}(t)^{2}+\ldots+\phi_{N, j}(t)^{2(l-1)}\right)\right. \\
& \left.\quad+\nu \sum_{i=1}^{N} \phi_{N, 1}(t)^{2 l-2}\right) .
\end{aligned}
$$

As

$$
\frac{1}{2^{l} N^{l+1}} \sum_{i, j=1 ; i \neq j}^{N} \phi_{N, i}(t)^{2(l-k)} \phi_{N, j}(t)^{2 k}=\frac{1}{2}\left(S_{N, l-k-1}(t) S_{N, k}(t)-\frac{S_{N, l-1}(t)}{N}\right)
$$

for $k=1,2, \ldots, l-2$, and as

$$
\frac{1}{2^{l} N^{l+1}} \sum_{i, j=1 ; i \neq j}^{N} \phi_{N, i}(t)^{2(l-1)}=\frac{N-1}{2 N} S_{N, l-1}(t),
$$

we obtain

$$
\frac{d}{d t} S_{N, l}(t)=l\left(\frac{2 N+\nu-l}{N} S_{N, l-1}(t)+\sum_{k=1}^{l-2} S_{N, l-1-k}(t) S_{N, k}(t)\right) .
$$

Similar to the Hermite case in Section 2, these equations show that the limits $c_{l}(t):=\lim _{N \rightarrow \infty} S_{N, l}(t)\left(l \in \mathbb{N}_{0}\right)$ exist and satisfy the following recurrence relation:

Lemma 4.3. Let $\left(x_{N, k}\right)_{1 \leq k \leq N} \subset \mathbb{R}$ be starting sequences such that for all $l \in \mathbb{N}_{0}$,

$$
c_{l}(0):=\lim _{N \rightarrow \infty} S_{N, l}(0)=\lim _{n \rightarrow \infty} \frac{1}{2^{l} N^{l+1}}\left(x_{N, 1}^{2 l}+\ldots+x_{N, N}^{2 l}\right)<\infty
$$

exists. Assume that $\nu=\nu(N)$ depends on $N$ with

$$
\lim _{N \rightarrow \infty} \frac{\nu(N)}{N}=\nu_{0} \geq 0
$$

Then for $l \in \mathbb{N}_{0}$,

$$
c_{l}(t):=\lim _{N \rightarrow \infty} S_{N, l}(t)
$$

exists locally uniformly in $t \in[0, \infty[$ and satisfies the recurrence relation

$c_{0}(t)=1 \quad$ and $\quad c_{l}(t)=c_{l}(0)+l \nu_{0} \int_{0}^{t} c_{l-1}(s) d s+l \int_{0}^{t} \sum_{k=0}^{l-1} c_{l-1-k}(s) c_{k}(s) d s \quad(l \geq 1)$. 
As in Section 2 we now show that under mild conditions on the starting sequences, the $c_{l}(t)$ are the moments of some unique probability measures which can be described via free probability.

We first consider the case $\nu_{0}=0$ which includes the case that all $\nu$ are independent of $N$. This special case can be easily reduced to Section 2 . We here denote the image of some probability measure $\mu$ under some continuous mapping $f$ by $f(\mu)$. We use this notation in particular for the maps $x \mapsto|x|$ and $x \mapsto x^{2}$ and write $|\mu|$ and $\mu^{2}$. Moreover, for a probability measure $\mu$ on $\left[0, \infty\left[\right.\right.$, let $\mu_{\text {even }}$ the unique even probability measure on $\mathbb{R}$ with $\left|\mu_{\text {even }}\right|=\mu$.

Theorem 4.4. Let $\mu \in M^{1}([0, \infty[)$ be a probability measure satisfying the moment condition 2.13). Let $\left(x_{N, n}\right)_{N \geq 1,1 \leq n \leq N} \subset\left[0, \infty\left[\right.\right.$ with $\left(x_{N, 1}, \ldots, x_{N, N}\right) \in C_{N}^{B}$ for $N \geq 2$ such that the normalized empirical measures

$$
\mu_{N, 0}:=\frac{1}{N}\left(\delta_{x_{N, 1} / \sqrt{2 N}}+\ldots \delta_{x_{N, N} / \sqrt{2 N}}\right)
$$

tend weakly to $\mu$ for $N \rightarrow \infty$. If we form the associated solutions $\phi_{N}(t)$ of (4.1) and the normalized empirical measures

$$
\mu_{N, t}:=\frac{1}{N}\left(\delta_{\phi_{N, 1}(t) / \sqrt{2 N}}+\ldots+\delta_{\phi_{N, N}(t) / \sqrt{2 N}}\right) \quad(t \geq 0),
$$

then for $t \in\left[0, \infty\left[\right.\right.$, the $\mu_{N, t}$ tend weakly to $\left|\mu_{s c, 2 \sqrt{t}} \boxplus \mu_{\text {even }}\right|$.

Proof. For $N \geq 2$ and $\left(x_{N, 1}, \cdots, x_{N, N}\right) \in C_{N}^{B}$ we define

$$
\left(y_{2 N, 1}, \cdots, y_{2 N, 2 N}\right)=\left(x_{N, 1}, \cdots, x_{N, N},-x_{N, N}, \cdots,-x_{N, 1}\right) \in C_{2 N}^{A} .
$$

Clearly, the associated empirical measures

$$
\mu_{N, \text { even }}:=\frac{1}{2 N}\left(\delta_{y_{2 N, 1} / \sqrt{2 N}}+\ldots+\delta_{y_{2 N, 1} / \sqrt{2 N}}\right)
$$

tend weakly to $\mu_{\text {even }}$. Moreover, all odd moments of $\mu_{\text {even }}$ disappear, and the even moments of $\mu_{\text {even }}$ and $\mu$ are equal. In particular, $\mu_{\text {even }}$ also satisfies the moment condition 2.13).

We now consider the even measures $\mu_{s c, 2 \sqrt{t}} \boxplus \mu_{\text {even }}$ whose odd moments disappear, and whose even moments satisfy (2.7) by Section 2. We obtain from (2.7) for these even moments and from 4.5 , that for all $l \geq 0$, the $c_{l}(t)$ from Lemma 4.3 are just the $2 l$-th moments of $\mu_{s c, 2 \sqrt{t}} \boxplus \mu_{\text {even }}$. This, Carleman's condition 2.12 for $\left(\mu_{s c, 2 \sqrt{t}} \boxplus \mu_{\text {even }}\right)^{2}$, Lemma 4.3 , and moment convergence now imply that the probability measures $\mu_{N, t}^{2}$ tend weakly to $\left(\mu_{s c, 2 \sqrt{t}} \boxplus \mu_{\text {even }}\right)^{2}$. This implies the claim.

If we start the ODE (4.1) in $x_{0}=0$, Theorem 4.4 and Lemma 4.2 lead to the following well known result for the empirical measures of the zeroes $z_{1, N}^{(\nu-1)}, \ldots, z_{N, N}^{(\nu-1)}$ of $L_{N}^{(\nu-1)}$; see e.g. $\mathrm{G}$ :

Corollary 4.5. Let $\nu>0$ be fixed. The empirical measures

$$
\mu_{N}:=\frac{1}{N}\left(\delta_{z_{1, N}^{(\nu-1)} /(4 N)}+\ldots+\delta_{z_{N, N}^{(\nu-1)} /(4 N)}\right)
$$

tend weakly to the beta distribution $\beta(1 / 2,3 / 2)$ on $[0,1]$ with the density

$$
\frac{2}{\pi} t^{-1 / 2}(1-t)^{1 / 2} \mathbf{1}_{] 0,1[}(t) \text {. }
$$


We now turn to the general case $\nu_{0} \geq 0$. We proceed similarly as in the Hermite case and deduce first a PDE for the Stieltjes transform $G$. This PDE will then be transformed into a PDE for the R-transform. Then we combine our results for $\nu_{0}=0$ and the R-transform for an appropriately parametrized Marchenko-Pastur distribution to identify the limit measure in general.

Recall that for the parameters $c \geq 0, t>0$, the Marchenko-Pastur distribution $\mu_{M P, c, t}$ is the probability measure on $\left[x_{-}, x_{+}\right] \subset\left[0, \infty\left[\right.\right.$ with $\mu_{M P, c, t}=\tilde{\mu}$ for $c \geq 1$ and $\mu_{M P, c, t}=(1-c) \delta_{0}+c \tilde{\mu}$ for $0 \leq c<1$, where $x_{ \pm}:=t(\sqrt{c} \pm 1)^{2}$ and $\tilde{\mu}$ has the Lebesgue density

$$
\frac{1}{2 \pi x t} \sqrt{\left(x_{+}-x\right)\left(x-x_{-}\right)} \cdot \mathbf{1}_{\left[x_{-}, x_{+}\right]}(x) .
$$

We also recall (see Exercise 5.3.27 of [AGZ]), that the Marchenko-Pastur distributions have the R-transforms

$$
R_{M P, c, t}(z)=\frac{c t}{1-t z} .
$$

As these R-transforms are linear in $c$, we in particular conclude that

$$
\mu_{M P, a, t} \boxplus \mu_{M P, b, t}=\mu_{M P, a+b, t} .
$$

Now we proceed as in Section 2. The proof of the first step is completely analog to Proposition 2.8 .

Proposition 4.6. Let $\lim _{N \rightarrow \infty} \nu(N) / N=\nu_{0} \geq 0$ in the setting of Lemma 4.3. Then the limits

$$
c_{l}(t):=\lim _{N \rightarrow \infty} S_{N, l}(t) \quad(t \in[0, \infty[, l \geq 0)
$$

exist. Moreover, if the moment condition (2.13) holds for $\mu^{2}$, then for each $t \in$ $\left[0, \infty\left[\right.\right.$, the sequence $\left(c_{l}(t)\right)_{l \geq 0}$ is the sequence of moments of some unique probability measure $\mu_{t}^{2} \in M^{1}(\mathbb{R})$ for which (2.13) also holds. Moreover, the $\mu_{N, t}^{2}$ tend weakly to $\mu_{t}^{2}$ for $N \rightarrow \infty$.

We next derive PDEs for the Stieltjes transforms

$$
G(t, z):=G_{\mu_{t}^{2}}(z), \quad G^{N}(t, z):=G_{\mu_{N, t}^{2}}(z) \quad(t \geq 0, z \in H=\{z \in \mathbb{C}: \Im z>0\})
$$

of the measures $\mu_{t}^{2}$ and $\mu_{N, t}^{2}$. In the setting of Proposition 4.6 we now have:

Proposition 4.7. (1) For all $N \in \mathbb{N}, t \geq 0, z \in H$,

$$
G_{t}^{N}(t, z)=-\frac{\nu(N)}{N} G_{z}^{N}(t, z)-2 z G_{z}^{N}(t, z) G^{N}(t, z)-\left(G^{N}\right)^{2}(t, z)-\frac{1}{N} E^{N}(t, z)
$$

with the error term $E^{N}(t, z)$ defined below in (4.11).

(2) Assume that in addition the moment condition (2.13) holds for $\mu^{2}$. Then for $t \geq 0, z \in H$,

$$
\begin{aligned}
G_{t}(t, z) & =-\nu_{0} G_{z}(t, z)-2 z G_{z}(t, z) G(t, z)-G^{2}(t, z) \\
G(0, z) & =G_{\mu}(z) .
\end{aligned}
$$

Proof. For $t \geq 0$ and $z \in H$ with $|z|$ sufficiently large (depending on $N$ ) we have

$$
G^{N}(t, z)=\int_{\mathbb{R}} \frac{1}{z-x} d \mu_{N, t}(x)=\sum_{l=0}^{\infty} \frac{S_{N, l}(t)}{z^{l+1}}
$$


and thus

$$
G_{t}^{N}(t, z)=\sum_{l=0}^{\infty} \frac{1}{z^{l+1}} \frac{d}{d t} S_{N, l}(t) .
$$

If we apply the recurrence relation (4.4) and the start (4.3), we obtain

$$
\begin{aligned}
G_{t}^{N}(t, z)= & \frac{N+\nu-1}{N} \cdot \frac{1}{z^{2}} \\
& +\sum_{l=2}^{\infty} \frac{1}{z^{l+1}} l\left(\frac{2 N+\nu-l}{N} S_{N, l-1}(t)+\sum_{k=1}^{l-2} S_{N, l-1-k}(t) S_{N, k}(t)\right) \\
= & \sum_{l=0}^{\infty} \frac{1}{z^{l+2}}(l+1) \sum_{k=0}^{l} S_{N, l-k}(t) S_{N, k}(t)+\frac{\nu}{N} \sum_{l=0}^{\infty}(l+1) \frac{1}{z^{l+2}} S_{N, l}(t) \\
& -\frac{1}{N} E^{N}(t, z)
\end{aligned}
$$

with

$$
E^{N}(t, z):=\sum_{l=0}^{\infty} \frac{(l+1)^{2}}{z^{l+2}} \cdot S_{N, l-2}(t) .
$$

Using as in the proof of 2.9 .

$$
z \cdot G_{z}^{N}(t, z)=-\sum_{l=0}^{\infty} \frac{l+1}{z^{l+1}} \cdot S_{N, l}(t), \quad z^{2} \cdot G_{z z}^{N}(t, z)=\sum_{l=0}^{\infty} \frac{(l+1)(l+2)}{z^{l+1}} \cdot S_{N, l}(t),
$$

we obtain by some simple calculation that

$$
E^{N}(t, z)=z G_{z z}^{N}(t, z)-G_{z}^{N}(t, z)
$$

As

$$
\begin{aligned}
\sum_{l=0}^{\infty} & \frac{1}{z^{l+2}}(l+1) \sum_{k=0}^{l} S_{N, l-k}(t) S_{N, k}(t)+\frac{\nu}{N} \sum_{l=0}^{\infty}(l+1) \frac{1}{z^{l+2}} S_{N, l}(t) \\
= & \sum_{l=0}^{\infty} \frac{1}{z^{l+2}}(l-k+1) \sum_{k=0}^{l} S_{N, l-k}(t) S_{N, k}(t)+\sum_{l=0}^{\infty} \frac{1}{z^{l+2}}(k+1) \sum_{k=0}^{l} S_{N, l-k}(t) S_{N, k}(t) \\
& \quad-\sum_{l=0}^{\infty} \frac{1}{z^{l+2}} \sum_{k=0}^{l} S_{N, l-k}(t) S_{N, k}(t)+\frac{\nu}{N} \sum_{l=0}^{\infty}(l+1) \frac{1}{z^{l+2}} S_{N, l}(t) \\
= & -\frac{\nu}{N} G_{z}^{N}(t, z)-2 z G_{z}^{N}(t, z) G^{N}(t, z)-\left(G^{N}\right)^{2}(t, z),
\end{aligned}
$$

part (1) follows for $z \in H$ sufficiently large. As both sides of the equation in part (1) are analytic in $z \in H$, this equation holds for all $z \in H$.

For (2) the arguments are the same as in the proof of (2.9).

Now we turn to the main result of this section:

Theorem 4.8. Let $\mu \in M^{1}\left(\left[0, \infty[)\right.\right.$ be a probability measure such that $\mu^{2}$ satisfies the moment condition 2.13). Let $\left(x_{N, n}\right)_{N \geq 1,1 \leq n \leq N} \subset\left[0, \infty\left[\right.\right.$ with $\left(x_{N, 1}, \ldots, x_{N, N}\right) \in$ $C_{N}^{B}$ such that the normalized empirical measures

$$
\mu_{N, 0}:=\frac{1}{N}\left(\delta_{x_{N, 1} / \sqrt{2 N}}+\ldots \delta_{x_{N, N} / \sqrt{2 N}}\right)
$$


tend weakly to $\mu$ for $N \rightarrow \infty$. For $N \geq 2$ we form the solutions $\phi_{N}$ of (4.1) with start in $\left(x_{N, 1}, \cdots, x_{N, N}\right)$. If

$$
\lim _{N \rightarrow \infty} \frac{\nu(N)}{N}=\nu_{0} \geq 0
$$

then for each $t \in[0, \infty[$, the associated normalized empirical measures

$$
\mu_{N, t}:=\frac{1}{N}\left(\delta_{\frac{\phi_{N, 1}(t)}{\sqrt{2 N}}}+\ldots+\delta_{\frac{\phi_{N, N}(t)}{\sqrt{2 N}}}\right) \quad(t \geq 0)
$$

tend weakly to $\sqrt{\mu_{M P, \nu_{0}, t} \boxplus\left(\mu_{s c, 2 \sqrt{t}} \boxplus \mu_{\text {even }}\right)^{2}}$.

Proof. We use (2.21), 2.22), and 2.23) in the PDE (4.10) and obtain

$$
R_{t}(t, G(t, z))=\nu_{0}+2 R(t, G(t, z)) \cdot G(t, z)+2-1+R_{z}(t, G(t, z)) \cdot G(t, z)^{2} .
$$

For $z$ instead of $G(t, z)$ we arrive at the following PDE for the R-transform:

$$
\begin{aligned}
R_{t}(t, z) & =\nu_{0}+1-2 z R(t, z)+z^{2} R_{z}(t, z) \\
R(0, z) & =R_{\mu}(z) .
\end{aligned}
$$

By Theorem 4.4 we know that $R_{\left(\mu_{s c, 2 \sqrt{t}} \boxplus \mu\right)^{2}}(z)$ solves 4.13 with $\nu_{0}=0$. This, 4.8, and a straight forward calculation now show that $R_{\mu_{M P, \nu_{0}, t}}(z)+R_{\left(\mu_{s c, 2 \sqrt{t}} \boxplus \mu_{e v e n}\right)^{2}}(z)$ solves the general PDE (4.13). This implies the desired result.

We finally consider Theorem 4.8 for the case with start in zero. As by a straight forward calculation $\left(\mu_{s c, 2 \sqrt{t}}\right)^{2}=\mu_{M P, 1, t}$, we here obtain the measures $\sqrt{\mu_{M P, 1+\nu_{0}, t}}$ as weak limits for the empirical measures $\mu_{N, t}$. This result and Lemma 4.2 on the zeroes $z_{1}^{(\nu(N)-1)}>\ldots>z_{N}^{(\nu(N)-1)}>0$ of $L_{N}^{(\nu(N)-1)}$ for $t=1 / 2$ lead to the following well known result (see e.g. [KM2] and references there):

Corollary 4.9. If $\lim _{N \rightarrow \infty} \nu(N) / N=\nu_{0} \geq 0$, then the empirical measures

$$
\frac{1}{N}\left(\delta_{z_{1}^{(\nu(N)-1) / 2 N}}+\ldots+\delta_{z_{N}^{(\nu(N)-1) / 2 N}}\right) \quad(t \geq 0),
$$

tend weakly to $\mu_{M P, 1+\nu_{0}, 1 / 2}$.

Theorem 4.8 has also the following consequence:

Corollary 4.10. For all $s, t \geq 0$ and $\nu_{0} \geq 0$,

$$
\mu_{M P, \nu_{0}, s} \boxplus\left(\mu_{s c, 2 \sqrt{s}} \boxplus\left(\sqrt{\mu_{M P, \nu_{0}+1, t}}\right)_{e v e n}\right)^{2}=\mu_{M P, \nu_{0}+1, s+t} .
$$

Proof. Theorem 4.8 with $\mu=\delta_{0}$ together with the semigroup property of our solutions of the ODEs 4.1) imply

$$
\begin{aligned}
\mu_{M P, \nu_{0}+1, s+t} & =\mu_{M P, \nu_{0}, s+t} \boxplus\left(\mu_{s c, 2 \sqrt{s+t}} \boxplus \delta_{0}\right)^{2} \\
& =\mu_{M P, \nu_{0}, s} \boxplus\left(\mu_{s c, 2 \sqrt{s}} \boxplus\left(\sqrt{\mu_{M P, \nu_{0}+1, t}}\right)_{\text {even }}\right)^{2} .
\end{aligned}
$$


This corollary, the additivity of the R-transform, and the known R-transforms of Marchenko-Pastur distributions lead immediately to the R-transform

$$
\begin{aligned}
R_{\left(\mu_{s c, 2 \sqrt{s}} \boxplus\left(\sqrt{\mu_{M P, \nu_{0}+1, t}}\right)_{e v e n}\right)^{2}} & =\frac{\left(\nu_{0}+1\right)(s+t)}{1-(s+t) z}-\frac{\nu_{0} s}{1-s z} \\
& =R_{\mu_{M P, 1, s+t}}(z)+\frac{\nu_{0} t}{(1-(s+t) z)(1-s z)} .
\end{aligned}
$$

\section{The Marchenko-Pastur law for Bessel processes of type B}

In this section we transfer the results for the ODEs (4.1) to a stochastic setting for the Bessel processes of type B. For this we consider Bessel processes $\left(X_{t, k}\right)_{t \geq 0}$ on the Weyl chambers $C_{N}^{B}$ for the root systems $B_{N}$ with $N \geq 2$ and multiplicities $k=\left(k_{1}, k_{2}\right)=(\nu \cdot \beta, \beta), \nu, \beta>0$. These processes satisfy the SDE

$$
d X_{t, k}^{i}=d B_{t}^{i}+\beta \sum_{j \neq i} \frac{X_{t, k}^{i}}{\left(X_{t, k}^{i}\right)^{2}-\left(X_{t, k}^{j}\right)^{2}} d t+\beta \frac{\nu}{X_{t, k}^{i}} d t \quad(i=1, \ldots, N) .
$$

with an $N$-dimensional Brownian motion $\left(B_{t}^{1}, \ldots, B_{t}^{N}\right)_{t \geq 0}$. By GrM we know, similar to Bessel processes of type $\mathrm{A}$, that for $\beta \geq 1 / 2$ and $\nu \beta \geq 1 / 2$, the process $\left(X_{t, k}\right)_{t>0}$ does not hit the boundary of $C_{N}^{B}$ a.s. for $t>0$ for arbitrary starting points in $C_{N}^{B}$. In the following we only consider this regular case $\beta \geq 1 / 2, \nu \beta \geq 1 / 2$. We now derive limit theorems for the moments of the associated empirical measures, where, as in the deterministic setting, we first consider the squares of all coordinates of our processes, namely

$$
\mu_{N, t}^{2}:=\frac{1}{N}\left(\delta_{\left(X_{t, k}^{1}\right)^{2} / 2 N}+\ldots+\delta_{\left(X_{t, k}^{N}\right)^{2} / 2 N}\right)
$$

for $t \geq 0$. For this it will be convenient also to study the renormalized processes $\left(\tilde{X}_{t, k}:=X_{t, k} / \sqrt{\beta}\right)_{t \geq 0}$ which satisfy the SDE

$$
d \tilde{X}_{t, k}^{i}=\frac{1}{\sqrt{\beta}} d B_{t}^{i}+\sum_{j \neq i} \frac{\tilde{X}_{t, k}^{i}}{\left(\tilde{X}_{t, k}^{i}\right)^{2}-\left(\tilde{X}_{t, k}^{j}\right)^{2}} d t+\frac{\nu}{\tilde{X}_{t, k}^{i}} d t \quad(i=1, \ldots, N) .
$$

which agrees, for $\beta=\infty$, with the ODE (4.1). Again we consider the renormalized empirical measures of the squares

$$
\tilde{\mu}_{N, t}^{2}:=\frac{1}{N}\left(\delta_{\left(\tilde{X}_{t, k}^{1}\right)^{2} / 2 N}+\ldots+\delta_{\left(\tilde{X}_{t, k}^{N}\right)^{2} / 2 N}\right) .
$$

Denote the $l$-th moment $\left(l \in \mathbb{N}_{0}\right)$ of $\tilde{\mu}_{N, t}$ by

$$
S_{N, l}(t):=\int_{\mathbb{R}} y^{l} d \tilde{\mu}_{N, t}(y)=\frac{1}{2^{l} N^{l+1}} \sum_{i=1}^{N}\left(\tilde{X}_{t, k}^{i}\right)^{2 l} .
$$

Now we derive limit theorems for these moments as $N \rightarrow \infty$. As in the deterministic setting the limits depend on the asymptotic behaviour of $\nu$.

Theorem 5.1. Consider the processes $\left(\tilde{X}_{t, k}\right)_{t \geq 0}$ with $\beta \geq 1 / 2, \nu>0$ and with starting sequences $\left(x_{N}\right)_{N \geq 1}:=\left(x_{N, n}\right)_{1 \leq k \leq N} \subset[0, \infty[$ as before such that the limits

$$
c_{l}(0):=\lim _{N \rightarrow \infty} S_{N, l}(0)=\lim _{N \rightarrow \infty} \frac{1}{2^{l} N^{l+1}}\left(x_{N, 1}^{2 l}+\ldots+x_{N, N}^{2 l}\right)<\infty
$$


exist for $l \geq 0$. Assume that $\nu=\nu(N)$ depends on $N$ with $\nu_{0}:=\lim _{N \rightarrow \infty} \nu(N) / N \geq$ 0 . Then, for $l \in \mathbb{N}_{0}$,

$$
c_{l}(t):=\lim _{N \rightarrow \infty} S_{N, l}(t)
$$

exists almost surely locally uniformly in $t \in\left[0, \infty\left[\right.\right.$ with $c_{0}(t)=1$ and

$$
c_{l}(t)=c_{l}(0)+l \nu_{0} \int_{0}^{t} c_{l-1}(s) d s+l \int_{0}^{t} \sum_{k=0}^{l-1} c_{l-1-k}(s) c_{k}(s) d s \quad(l \geq 1) .
$$

Proof. Using Itô's formula we obtain for $l \geq 1$

$$
\begin{aligned}
& \sum_{i=1}^{N}\left(\tilde{X}_{t, k}^{i}\right)^{2 l}=\sum_{i=1}^{N} x_{i}^{2 l}+\frac{2 l}{\sqrt{\beta}} \sum_{i=1}^{N} \int_{0}^{t}\left(\tilde{X}_{s, k}^{i}\right)^{2 l-1} d B_{s}^{i} \\
& \quad+\int_{0}^{t}\left(2 l \sum_{i=1}^{N} \sum_{j \neq i} \frac{2\left(\tilde{X}_{s, k}^{i}\right)^{2 l}}{\left(\tilde{X}_{s, k}^{i}\right)^{2}-\left(\tilde{X}_{s, k}^{j}\right)^{2}}+\sum_{i=1}^{N}\left(2 l \nu+\frac{l(2 l-1)}{\beta}\right)\left(\tilde{X}_{s, k}^{i}\right)^{2 l-2}\right) d s
\end{aligned}
$$

Now we see that for the normalized drift term we obtain the recurrence relation 4.4 , where $\nu$ is replaced by $\nu+\frac{2 l-1}{2 \beta}$. The desired results now follow along the same lines as in the proofs of Lemma 3.1. Corollary 3.3, and Theorem 3.4 using the deterministic results of (4.3), as well as Theorem 4.8. We here skip the details, as the details are part of the more complicated setting in Section 8.

The methods of Section 3 and Theorem 5.1 lead to the following limit theorem.

Theorem 5.2. Let $\mu \in M^{1}\left(\left[0, \infty[)\right.\right.$ such that $\mu^{2}$ satisfies the moment condition 2.13). Let $\left(x_{N, n}\right)_{N \geq 1,1 \leq n \leq N} \subset\left[0, \infty\left[\right.\right.$ with $\left(x_{N, 1}, \ldots, x_{N, N}\right) \in C_{N}^{B}$ such that the empirical measures

$$
\mu_{N, 0}:=\frac{1}{N}\left(\delta_{x_{N, 1} / \sqrt{2 N}}+\ldots \delta_{x_{N, N} / \sqrt{2 N}}\right)
$$

tend weakly to $\mu$ for $N \rightarrow \infty$. Consider the normalized Bessel processes $\left(\tilde{X}_{t, k}\right)_{t \geq 0}$ of type $B$ with start in $\left(x_{N, 1}, \ldots, x_{N, N}\right) \in C_{N}^{B}$ for $N \geq 2$. Then, for $t \geq 0$ and

$$
\lim _{N \rightarrow \infty} \frac{\nu(N)}{N}=\nu_{0} \geq 0
$$

the normalized empirical measures

$$
\mu_{N, t}:=\frac{1}{N}\left(\delta_{\frac{\tilde{x}_{t, k}^{1}}{\sqrt{2 N}}}+\ldots+\delta_{\frac{\tilde{X}_{t, k}^{N}}{\sqrt{2 N}}}\right)
$$

tend a.s. weakly to $\sqrt{\mu_{M P, \nu_{0}, t} \boxplus\left(\mu_{s c, 2 \sqrt{t}} \boxplus \mu_{\text {even }}\right)^{2}}$ for $N \rightarrow \infty$.

\section{DUNKL PROCESSES AND THEIR FROZEN VERSIONS}

In the remainder of the paper we extend the results of Sections 4 and 5 for Bessel processes of type B and their frozen versions to Dunkl processes of type B. As already discussed in the introduction, this extension from Bessel to Dunkl processes does not lead to new limit results for the root systems of type A. Nevertheless, we recapitulate briefly some facts on general Dunkl processes for the convenience of the reader. Moreover, the frozen versions do not appear in the literature. To keep the discussion concise we assume that the reader is familiar with root systems, Weyl 
groups, and Weyl chambers for the general theory. On the other hand, we present all details for the root systems of type B, as we are interested mainly in this case.

All results on Dunkl theory and Dunkl processes and the algebraic and analytic background can be found in [An, CGY, R1, R2, RV1, RV2] and references there.

Let $R \subset \mathbb{R}^{N}$ be a root system and $R_{+} \subset R$ a subsystem consisting of positive roots. We assume that all roots $\alpha \in R$ satisfy $\|\alpha\|_{2}^{2}=2$. Then, for all $\alpha \in R$, the reflection $\sigma_{\alpha}$ on the hyperplane perpendicular to $\alpha$ is given by $\sigma_{\alpha}(x)=x-(\alpha \cdot x) \alpha$ for $x \in \mathbb{R}^{N}$ with the standard scalar product $\cdot$ on $\mathbb{R}^{N}$. Let $W \subset O(N)$ be the finite reflection group, or Weyl group, generated by the reflections $\sigma_{\alpha}, \alpha \in R_{+}$. The Weyl group $W$ acts on $\mathbb{R}^{N}$ as usual, and we have $W(R)=R$. We next fix some nonnegative multiplicity function $k: R \rightarrow[0, \infty[$ which is by definition invariant under the canonical action of $W$ on $R$.

For given $R$ and $k$ we now define the Dunkl operators $T_{i}(i=1, \ldots, N)$ as the differential-difference operators

$$
T_{i} f(x):=\frac{\partial f(x)}{\partial x_{i}}+\sum_{\alpha \in R_{+}} k(\alpha) \alpha_{i} \frac{f(x)-f\left(\sigma_{\alpha}(x)\right)}{\alpha \cdot x} \quad\left(f \in C^{2}\left(\mathbb{R}^{N}\right)\right) .
$$

It is well known by Dunkl (see e.g. [DX]) that the operators $T_{i}$ commute with $T_{i} f \in C^{1}\left(\mathbb{R}^{N}\right)$ for $f \in C^{2}\left(\mathbb{R}^{N}\right)$. Moreover, the $T_{i}$ lower the degree of homogeneous polynomials in $N$ variables by one like the usual partial derivative operators, which appear as special cases for $k=0$.

We next introduce the Dunkl Laplacian $L_{k}:=\sum_{i=1}^{N} T_{i}^{2}$ as well as its renormalization $\mathcal{L}_{k}:=\frac{1}{2} L_{k}$, which fits better to the usual normalization of Brownian motions in probability. $\mathcal{L}_{k}$ is given explicitly by

$$
\mathcal{L}_{k} f(x)=\frac{1}{2} \Delta f(x)+\sum_{\alpha \in R_{+}} k(\alpha)\left(\frac{\nabla f(x) \cdot \alpha}{\alpha \cdot x}+\frac{f\left(\sigma_{\alpha}(x)\right)-f(x)}{(\alpha \cdot x)^{2}}\right)
$$

for $f \in C_{c}^{2}\left(\mathbb{R}^{N}\right)$ with the usual Laplacian $\Delta$ on $\mathbb{R}^{N}$; see e.g. [CGY, RV2]. The operator $\mathcal{L}_{k}$ is the generator of some Feller semigroup on $\mathbb{R}^{N}$ whose transition densities can be written down explicitly in terms of so-called Dunkl kernels; see [R1, RV1 for details. Associated Feller processes $\left(X_{t, k}\right)_{t \geq 0}$ on $\mathbb{R}^{N}$ with càdlàg paths are called Dunkl processes associated with the root system $R$ with multiplicity $k$.

Let us briefly discuss the corresponding Bessel processes. For this we fix some closed Weyl chamber associated with $R$. This chamber may be regarded as the space $\mathbb{R}^{N} / W$ of all $W$-orbits in $\mathbb{R}^{N}$, i.e., for each $x \in \mathbb{R}^{N}$ there is some unique $\pi(x) \in C$ for some $\pi \in W$. We thus have some canonical projection $\pi: \mathbb{R}^{N} \rightarrow \mathbb{R}^{N} / W \simeq C$, and we can consider the projected processes

$$
\left(X_{t, k}^{W}:=\pi\left(X_{t, k}\right)\right)_{t \geq 0}
$$

on $C$. By symmetry arguments, these processes $\left(X_{t, k}^{W}\right)_{t \geq 0}$ are time-homogeneous diffusion processes on $C$ with reflecting boundaries and with the generators

$$
\mathcal{L}_{k}^{W} f(x)=\frac{1}{2} \Delta f(x)+\sum_{\alpha \in R_{+}} k(\alpha) \frac{\nabla f(x) \cdot \alpha}{\alpha \cdot x}
$$

for functions $f \in C_{c}^{2}\left(\mathbb{R}^{N}\right)$ which are $W$-invariant. Notice that this restriction of the domain of $\mathcal{L}_{k}^{W}$ fits to the reflecting boundaries. These diffusions on $C$ are called Bessel processes associated with the root system $R$ with multiplicity $k$.

We now discuss examples which are connected with the preceding sections: 
Examples 6.1. (1) For $N \geq 2$, the root system of type $A_{N-1}$ is given by

$$
R=\left\{e_{i}-e_{j}: \quad i, j=1, \ldots, N, i \neq j\right\}
$$

where $e_{i} \in \mathbb{R}^{N}$ is the $i$-th unit vector. Here, $W$ is the symmetric group acting on $\mathbb{R}^{N}$ as usual, the multiplicity $k$ is just a constant, the Weyl chamber $C$ may be chosen as $C_{N}^{A}$ from the introduction, and the generator in 6.2 is just the generator of the Bessel process of type $A_{N-1}$ as in 1.2 .

(2) For $N \geq 1$, the root system of type $B_{N}$ is given by

$$
R=\left\{ \pm\left(e_{i} \pm e_{j}\right): \quad 1 \leq i<j \leq N\right\} \cup\left\{ \pm \sqrt{2} \cdot e_{i}: \quad i=1, \ldots, N\right\} .
$$

Here, $W$ is the hyperoctahedral group $\mathbb{Z}_{2}^{N} \ltimes S_{N}$, the multiplicity $k$ consists of 2 constants, the Weyl chamber $C$ may be chosen as $C_{N}^{B}$ from the introduction, and the generator in $(6.2)$ is just the generator of the Bessel process of type $B_{N}$ introduced in $(1.5)$.

We now consider the freezing of Dunkl processes similar to that of Bessel processes in the preceding sections. For this we fix some root system $R$ and write the multiplicity function $k: R \rightarrow\left[0, \infty\left[\right.\right.$ as $k=\beta \cdot k_{0}$ with some fixed multipicity function $k_{0}$ and a varying constant $\beta>0$. For an Dunkl process $\left(X_{t, k}\right)_{t>0}$ on $\mathbb{R}^{N}$ associated

with $R$ and $k$, we now also study its renormalized version $\left(\tilde{X}_{t, k}:=\frac{1}{\sqrt{\beta}} X_{t, k}\right)_{t \geq 0}$ which then has the generator

$$
\tilde{\mathcal{L}}_{k_{0}, \beta} f(x)=\frac{1}{2 \beta} \Delta f(x)+\sum_{\alpha \in R_{+}} k_{0}(\alpha)\left(\frac{\nabla f(x) \cdot \alpha}{\alpha \cdot x}+\frac{f\left(\sigma_{\alpha}(x)\right)-f(x)}{(\alpha \cdot x)^{2}}\right)
$$

for $f \in C_{c}^{2}\left(\mathbb{R}^{N}\right)$. Clearly, this is also the generator of some Feller semigroup for $\beta=\infty$. Associated Feller processes then will be called frozen Dunkl processes associated with $R$ and $k_{0}$. These processes are pure jump processes.

Example 6.2. In the next section we study frozen Dunkl processes for the root system $B_{N}$. Here we choose the fixed multiplicity $k_{0}$, which consists of 2 parameters, as $k_{0}=(1, \nu)$ with $\nu \geq 0$. In this case, we denote the frozen Dunkl processes on $\mathbb{R}^{N}$ by $\left(X_{t, \nu}\right)_{t \geq 0}$. The associated generator $\tilde{\mathcal{L}}_{k_{0}, \infty}$ according to 6.3 will now be denoted as $L_{\nu}$ for simplicity. We then have

$$
\begin{aligned}
L_{\nu} u(x) & =\sum_{i=1}^{N}\left(\sum_{j: j \neq i} \frac{2 x_{i}}{x_{i}^{2}-x_{j}^{2}}+\frac{\nu}{x_{i}}\right) u_{x_{i}}(x)+\frac{\nu}{2} \sum_{i=1}^{N} \frac{u\left(\sigma_{i} x\right)-u(x)}{x_{i}^{2}} \\
& +\frac{1}{2} \sum_{i, j: j \neq i}\left(\frac{u\left(\sigma_{i, j} x\right)-u(x)}{\left(x_{i}-x_{j}\right)^{2}}+\frac{u\left(\sigma_{i, j}^{-} x\right)-u(x)}{\left(x_{i}+x_{j}\right)^{2}}\right)
\end{aligned}
$$

for $u \in \mathcal{C}^{2}\left(\mathbb{R}^{N}\right)$ with the reflections $\sigma_{i}, \sigma_{i, j}, \sigma_{i, j}^{-}(i \neq j)$ on $\mathbb{R}^{N}$ where $\sigma_{i}$ changes the sign of the $i$-th coordinate, $\sigma_{i, j}$ exchanges the coordinates $i, j$, and $\sigma_{i, j}^{-}$exchanges the coordinates $i, j$ and changes the signs of these coordinates in addition.

The Dunkl Laplacians $\tilde{\mathcal{L}}_{k_{0}, \beta}$ from $(6.3)$ for the root system $B_{N}$ with $k_{0}=(1, \nu)$ will be denoted by $\tilde{\mathcal{L}}_{\nu, \beta}$ in the next sections.

\section{Empirical Limit distributions for frozen Dunkl Processes of type B}

In this section we study the empirical distributions of frozen Dunkl processes $\left(X_{t, \nu}\right)_{t \geq 0}$ of type $B$ on $\mathbb{R}^{N}$ with the generators $L_{\nu}$ defined in 6.4. We assume 
that these processes start in deterministic points in $\mathbb{R}^{N}$. Moreover, we denote the components of $X_{t, \nu}$ by $X_{j, t, \nu}$ for $j=1, \ldots, N$.

Similar to Sections 2 and 4 we study the (random) normalized empirical measures

$$
\mu_{N, t, \nu}:=\frac{1}{N}\left(\delta_{X_{1, t, \nu} / \sqrt{N}}+\ldots+\delta_{X_{N, t, \nu} / \sqrt{N}}\right) \in M^{1}(\mathbb{R})
$$

of the processes $\left(X_{t, \nu}\right)_{t \geq 0}$ of dimension $N$ for $N \rightarrow \infty$. For this we study their moments

$$
S_{N, l, \nu}(t):=\frac{1}{N^{l / 2+1}}\left(X_{1, t, \nu}^{l}+\ldots+X_{N, t, \nu}^{l}\right) \quad(l \geq 0)
$$

By the very construction of the processes $\left(X_{t, \nu}\right)_{t \geq 0}$, the even moments $S_{N, 2 l, \nu}(t)$ are deterministic (and closely related to corresponding moments of the corresponding frozen Bessel processes of type B in Section 4), while this is not the case for the odd moments $S_{N, 2 l+1, \nu}(t)(l \geq 0)$. With the functions

$$
u_{l}(x):=x_{1}^{l}+\ldots+x_{N}^{l} \quad(l \geq 0)
$$

we have

$$
S_{N, l, \nu}(t)=\frac{1}{N^{1+l / 2}} \cdot u_{l}\left(X_{t, \nu}\right) \quad(l \geq 0) .
$$

We now compute $L_{\nu} u_{l}$ for $l \geq 0$. For this we observe that all $u_{l}$ are invariant under permutations of coordinates. Therefore, for all $u:=u_{l}$

$$
\begin{aligned}
L_{\nu} u(x) & =\sum_{i=1}^{N}\left(\sum_{j: j \neq i} \frac{2 x_{i}}{x_{i}^{2}-x_{j}^{2}}+\frac{\nu}{x_{i}}\right) u_{x_{i}}(x)+\frac{\nu}{2} \sum_{i=1}^{N} \frac{u\left(\sigma_{i} x\right)-u(x)}{x_{i}^{2}} \\
& +\frac{1}{2} \sum_{i, j: j \neq i} \frac{u\left(\sigma_{i, j}^{-} x\right)-u(x)}{\left(x_{i}+x_{j}\right)^{2}} .
\end{aligned}
$$

Moreover, Dynkin's formula for Markov processes (see e.g. Section III.10 of [RW]) shows that the processes

$$
\left(u_{l}\left(X_{t, \nu}\right)-u_{l}\left(X_{0, \nu}\right)-\int_{0}^{t}\left(L_{\nu} u_{l}\right)\left(X_{s, \nu}\right) d s\right)_{t \geq 0}
$$

are martingales. Hence, for all $l \geq 0$,

$$
\frac{d}{d t} E\left(u_{l}\left(X_{t, \nu}\right)\right)=E\left(\left(L_{\nu} u_{l}\right)\left(X_{t, \nu}\right)\right)
$$

We now compute $L_{\nu} u_{l}$ for $l \geq 0$. We first notice that $u_{0}=N$ and thus $L_{\nu} u_{0}=$ 0 . Moreover, a simple computation yields that $L_{\nu} u_{1}=0$. By Dynkin's formula 7.5 this corresponds to the well-known fact that the process $\left(u_{1}\left(X_{t, \nu}\right)\right)_{t \geq 0}$ is a martingale; see e.g. RV1]. We next turn to the general odd case $u_{2 l+1}$ for $l \geq 1$. 
By (7.4) we have

$$
\begin{aligned}
L_{\nu} u_{2 l+1}(x)= & \sum_{i=1}^{N}\left(\sum_{j: j \neq i} \frac{2 x_{i}^{2 l+1}}{x_{i}^{2}-x_{j}^{2}}+\nu x_{i}^{2 l-1}\right)(2 l+1) \\
& -\sum_{i, j: j \neq i} \frac{x_{i}^{2 l+1}+x_{j}^{2 l+1}}{\left(x_{i}+x_{j}\right)^{2}}-\nu \sum_{i=1}^{N} x_{i}^{2 l-1} \\
= & (2 l+1) \sum_{i, j: j \neq i} \frac{x_{i}^{2 l+1}-x_{j}^{2 l+1}}{x_{i}^{2}-x_{j}^{2}}-\sum_{i, j: j \neq i} \sum_{h=0}^{2 l}(-1)^{h} \frac{x_{i}^{h} x_{j}^{2 l-h}}{x_{i}+x_{j}} \\
& +(2 l+1) \nu u_{2 l-1}(x)-\nu u_{2 l-1}(x)
\end{aligned}
$$

and thus

$$
\begin{aligned}
L_{\nu} u_{2 l+1}(x)= & (2 l+1) \sum_{i, j: j \neq i} \sum_{h=0}^{2 l} \frac{x_{i}^{h} x_{j}^{2 l-h}}{x_{i}+x_{j}} \\
& -\sum_{i, j: j \neq i} \sum_{h=0}^{2 l}(-1)^{h} \frac{x_{i}^{h} x_{j}^{2 l-h}}{x_{i}+x_{j}}+2 l \nu u_{2 l-1}(x) \\
= & \sum_{i, j: j \neq i} \frac{1}{x_{i}+x_{j}}\left(2 l \sum_{h=0}^{l} x_{i}^{2 h} x_{j}^{2 l-2 h}+2(l+1) \sum_{h=0}^{l-1} x_{i}^{2 h+1} x_{j}^{2 l-2 h-1}\right) \\
& +2 l \nu u_{2 l-1}(x) \\
= & \sum_{i, j: j \neq i}\left(2 l x_{j}^{2 l-1}+2 x_{j}^{2 l-2} x_{i}+(2 l-2) x_{j}^{2 l-3} x_{i}^{2}+4 x_{j}^{2 l-4} x_{i}^{3}+\ldots\right. \\
& \left.\ldots+2 x_{j} x_{i}^{2 l-2}+2 l x_{i}^{2 l-1}\right)+2 l \nu u_{2 l-1}(x) \\
= & 2\left(\sum_{i, j: j \neq i} \sum_{h=0}^{l-1} x_{i}^{2 h} x_{j}^{2 l-1-2 h}(l-h)+\sum_{i, j: j \neq i} \sum_{h=0}^{l-1} x_{j}^{2 h} x_{i}^{2 l-1-2 h}(l-h)\right) \\
& +2 l \nu u_{2 l-1}(x) \\
= & 4 \sum_{i, j: j \neq i} \sum_{h=0}^{l-1}(l-h) x_{i}^{2 h} x_{j}^{2 l-1-2 h}+2 l \nu u_{2 l-1}(x) .
\end{aligned}
$$

As $\sum_{i, j: j \neq i} x_{i}^{2 h} x_{j}^{2 l-1-2 h}=u_{2 h}(x) u_{2 l-1-2 h}(x)-u_{2 l-1}(x), \sum_{h=0}^{l-1}(l-h)=l(l+1) / 2$ and $u_{0}=N$, we get

$$
\begin{aligned}
L_{\nu} u_{2 l+1} & =2 l \nu u_{2 l-1}+4 \sum_{h=0}^{l-1}(l-h) u_{2 h} u_{2 l-1-2 h}-4 \sum_{h=0}^{l-1}(l-h) u_{2 l-1} \\
& =(2 l \nu+4 l N-2 l(l+1)) u_{2 l-1}+4 \sum_{h=1}^{l-1}(l-h) u_{2 h} u_{2 l-1-2 h} \\
& =2 l(2 N+\nu-(l+1)) u_{2 l-1}+4 \sum_{h=1}^{l-1}(l-h) u_{2 h} u_{2 l-1-2 h} .
\end{aligned}
$$


Moreover, by a similar computation we obtain in the even case for $l \geq 1$ that

$$
L_{\nu} u_{2 l}=2 l(\nu+l+1) u_{2 l-2}+2 l \sum_{h=0}^{l-1} u_{2 h} u_{2 l-2-2 h} .
$$

If we combine 7.6 with 7.8 and use that $u_{2 h}\left(X_{t, \nu}\right)$ is deterministic, we see that

$$
\begin{array}{rl}
\frac{d}{d t} E\left(u_{2 l+1}\left(X_{t, \nu}\right)\right)=E & E\left(\left(L_{\nu} u_{2 l+1}\right)\left(X_{t, \nu}\right)\right) \\
=2 & 2 l(2 N+\nu-(l+1)) E\left(u_{2 l-1}\left(X_{t, \nu}\right)\right) \\
& +4 \sum_{h=1}^{l-1}(l-h) u_{2 h}\left(X_{t, \nu}\right) \cdot E\left(u_{2 l-1-2 h}\left(X_{t, \nu}\right)\right) .
\end{array}
$$

Therefore, by (7.3),

$$
\begin{aligned}
\frac{d}{d t} E\left(S_{N, 2 l+1, \nu}(t)\right)= & \frac{2 l(2 N+\nu-(l+1))}{N} E\left(S_{N, 2 l-1, \nu}(t)\right) \\
& +4 \sum_{h=1}^{l-1}(l-h) S_{N, 2 h, \nu}(t) E\left(S_{N, 2 l-1-2 h, \nu}(t)\right) .
\end{aligned}
$$

Moreover, in the even case we obtain in a similar way from $(7.9)$ that

$$
\frac{d}{d t} S_{N, 2 l, \nu}(t)=\frac{2 l(\nu+l+1)}{N} S_{N, 2 l-2, \nu}(t)+2 l \sum_{h=0}^{l-1} S_{N, 2 l-2-2 h, \nu}(t) S_{N, 2 h, \nu}(t) .
$$

These two recurrence relation and the methods of the proofs of Lemmas 2.4 and 4.3 now readily lead to the following limit result.

Lemma 7.1. Let $\left(x_{N, k}\right)_{1 \leq k \leq N} \subset \mathbb{R}$ be the starting sequences of the frozen Dunkl processes $\left(X_{t, \nu}\right)_{t \geq 0}$ for $N \in \mathbb{N}$ with $X_{0, \nu}=\left(x_{N, 1}, \ldots, x_{N, N}\right)$ such that

$$
c_{l}(0):=\lim _{N \rightarrow \infty} S_{N, l, \nu}(0)=\lim _{n \rightarrow \infty} \frac{1}{N^{l / 2+1}}\left(x_{N, 1}^{l}+\ldots+x_{N, N}^{l}\right)<\infty
$$

exists for all $l \in \mathbb{N}_{0}$. Assume that $\nu=\nu(N)$ depends on $N$ with $\lim _{N \rightarrow \infty} \frac{\nu(N)}{N}=\nu_{0}$ with some constant $\nu_{0} \geq 0$. Then for $l \in \mathbb{N}_{0}$,

$$
c_{l}(t):=\lim _{N \rightarrow \infty} E\left(S_{N, l, \nu(N)}(t)\right)
$$

exists locally uniformly in $t \in\left[0, \infty\left[\right.\right.$ and satisfies the recurrence relations $c_{0}(t)=1$, $c_{1}(t)=c_{1}(0)$, and for $l \geq 1$,

$$
\begin{gathered}
c_{2 l}(t)=c_{2 l}(0)+2 l \int_{0}^{t}\left(\nu_{0} c_{2 l-2}(s)+\sum_{h=0}^{l-1} c_{2 h}(s) c_{2 l-2 h-2}(s)\right) d s \\
c_{2 l+1}(t)=c_{2 l+1}(0)+\int_{0}^{t}\left(2 l \nu_{0} c_{2 l-1}(s)+4 \sum_{h=0}^{l-1}(l-h) c_{2 h}(s) c_{2 l-2 h-1}(s)\right) d s
\end{gathered}
$$

Please notice that the even limit moments $c_{2 l}(t)$ in 7.12 have the same recurrence relation as the moments $c_{l}(t)$ in 4.5 in the context of Bessel processes of type B up to an factor 2, This factor 2 is caused by by the slightly different scalings of the emirical measures in (7.1) and (4.2).

We now proceed as in Sections 2 and 4 and obtain that under some mild conditions on the initial data, the $c_{l}(t)(l \geq 0)$ in the lemma are the moments of 
unique probability measures $\mu_{t}$ for $t \geq 0$. For this we fix some probability measure $\mu \in M^{1}(\mathbb{R})$ which is determined uniquely by its moments $c_{l}(l \geq 0)$. We now choose $\left(x_{N, n}\right)_{N \geq 1,1 \leq n \leq N} \subset \mathbb{R}$ with $x_{N, n-1} \geq x_{N, n}$ for $2 \leq n \leq N$ such that the empirical measures

$$
\mu_{N, 0}:=\frac{1}{N}\left(\delta_{x_{N, 1} / \sqrt{N}}+\ldots \delta_{x_{N, N} / \sqrt{N}}\right)
$$

tend weakly to $\mu$ for $N \rightarrow \infty$, i.e., by the moment convergence theorem, that

$$
\lim _{N \rightarrow \infty} S_{N, l, \nu}(0):=\lim _{N \rightarrow \infty} \frac{1}{N^{l / 2+1}}\left(x_{N, 1}^{l}+\ldots+x_{N, N}^{l}\right)=c_{l} \quad(l \geq 0) .
$$

For $N \geq 2$ we now consider the measures $\mu_{N, t, \nu}$ from $(7.1$. The proof of the following result is now completely analogous to that of Propositions 2.8 or 4.6

Proposition 7.2. Let $\mu \in M^{1}(\mathbb{R})$ be a probability measure for which the moment condition (2.13) holds. Assume that with the preceding notations $\lim _{N \rightarrow \infty} \frac{\nu(N)}{N}=$ $\nu_{0} \geq 0$ exists. Then for $t \geq 0$ there are unique probability measures $\mu_{t} \in M^{1}(\mathbb{R})$ with the moments $\left(c_{l}(t)\right)_{l \geq 0}$. Moreover, for all $t \geq 0$, the moment condition (2.13) holds for $\mu_{t}$, and $\mu_{t}$ is the weak limit of the empirical measures $\mu_{N, t, \nu(N)}$ for $N \rightarrow \infty$.

In the situation of Proposition 7.2 we now derive PDEs for the Stieltjes transforms

$$
G(t, z):=G_{\mu_{t}}(z), \quad G^{N}(t, z):=G_{\mu_{N, t, \nu}(N)}(z) \quad(t \geq 0, z \in \mathbb{C}, \Im z \neq 0)
$$

of the measures $\mu_{t}$ and $\mu_{N, t, \nu(N)}$. As the recurrence relations in Lemma 7.1 are different for even and odd moments, we decompose $\mu_{t}$ and $\mu_{N, t, \nu(N)}$ into their even and odd parts and study the Stieltjes transforms of these measures. We thus define the reflected probability measures $\mu_{t}^{*} \in M^{1}(\mathbb{R})$ with $\mu_{t}^{*}(A)=\mu_{t}(-A)$ for Borel sets $A \subset \mathbb{R}$ and

$$
\mu_{t, \text { even }}:=\frac{1}{2}\left(\mu_{t}+\mu_{t}^{*}\right), \quad \mu_{t, \text { odd }}:=\frac{1}{2}\left(\mu_{t}-\mu_{t}^{*}\right) .
$$

Please notice that $\mu_{t, \text { odd }}$ usually is a signed measure, and that $\mu_{t}=\mu_{t, \text { even }}+\mu_{t, \text { odd }}$. We now introduce the Stieltjes transforms

$$
G^{\text {even }}(t, z):=G_{\mu_{t, \text { even }}}(z), \quad G^{o d d}(t, z):=G_{\mu_{t, \text { odd }}}(z)
$$

with $G=G^{\text {even }}+G^{\text {odd }}$. As by the definition 2.17 of the Stieltjes transform $G_{\mu_{t}^{*}}(z)=-G_{\mu_{t}}(-z)$, and as $G_{\mu_{t}}(z)=\sum_{l=0}^{\infty} c_{l}(t) z^{-(l+1)}$ for $|z|$ sufficiently large, we obtain that

$$
\begin{aligned}
G^{\text {even }}(t, z) & =\frac{1}{2}(G(t, z)-G(t,-z))=\sum_{l=0}^{\infty} \frac{c_{2 l}(t)}{z^{2 l+1}}=z \tilde{G}^{\text {even }}\left(t, z^{2}\right) \\
G^{\text {odd }}(t, z) & =\frac{1}{2}(G(t, z)+G(t,-z))=\sum_{l=0}^{\infty} \frac{c_{2 l+1}(t)}{z^{2 l+2}}=\tilde{G}^{\text {odd }}\left(t, z^{2}\right)
\end{aligned}
$$

with the functions

$$
\tilde{G}^{\text {even }}(t, z):=\sum_{l=0}^{\infty} \frac{c_{2 l}(t)}{z^{l+1}}, \quad \tilde{G}^{\text {odd }}(t, z):=\sum_{l=0}^{\infty} \frac{c_{2 l+1}(t)}{z^{l+1}} .
$$

We use the corresponding notations and relations also for the measures $\mu_{N, t, \nu(N)}$.

In the setting of Proposition 7.2 we now have: 
Proposition 7.3. The Stieltjes transforms $G^{\text {even }}, G^{\text {odd }}$ and the functions $\tilde{G}^{\text {even }}, \tilde{G}^{\text {odd }}$ satisfy the PDEs

$$
\begin{aligned}
\tilde{G}_{t}^{\text {even }}(t, z) & =-2 \nu_{0} \tilde{G}_{z}^{\text {even }}(t, z)-4 z \tilde{G}_{z}^{\text {even }}(t, z) \tilde{G}^{\text {even }}(t, z)-2 \tilde{G}^{\text {even }}(t, z)^{2}, \\
\tilde{G}_{t}^{\text {odd }}(t, z) & =\left(-2 \nu_{0}-4 z \tilde{G}^{\text {even }}(t, z)\right) \tilde{G}_{z}^{\text {odd }}(t, z)
\end{aligned}
$$

and

$$
\begin{aligned}
G_{t}^{\text {even }}(t, z) & =\nu_{0}\left(\frac{G^{\text {even }}(t, z)}{z^{2}}-\frac{G_{z}^{\text {even }}(t, z)}{z}\right)-2 G^{\text {even }}(t, z) G_{z}^{\text {even }}(t, z) \\
G_{t}^{\text {odd }}(t, z) & =\left(-\frac{\nu_{0}}{z}-2 G^{\text {even }}(t, z)\right) G_{z}^{\text {odd }}(t, z)
\end{aligned}
$$

for $t \geq 0, z \in H$.

Proof. We first consider $\tilde{G}^{\text {even }}$. Here, 7.15, $\frac{d}{d t} c_{0}(t)=0$, and 7.12 imply that

$$
\begin{aligned}
\frac{d}{d t} \tilde{G}^{\text {even }}(t, z) & =\sum_{l=1}^{\infty} \frac{1}{z^{l+1}} \frac{d}{d t} c_{2 l}(t)=\sum_{l=0}^{\infty} \frac{1}{z^{l+2}} \frac{d}{d t} c_{2 l+2}(t) \\
& =\sum_{l=0}^{\infty} \frac{1}{z^{l+2}}\left(2(l+1) \nu_{0} c_{2 l}(t)+2 \sum_{h=0}^{l}(l+1) c_{2 h}(t) c_{2 l-2 h}(t)\right) \\
& =-2 \nu_{0} \tilde{G}_{z}^{\text {even }}(t, z)-2 \frac{d}{d z}\left(z \cdot \tilde{G}^{\text {even }}(t, z)^{2}\right)
\end{aligned}
$$

which implies the first equation in 7.16 . Moreover, $7.15, \frac{d}{d t} c_{1}(t)=0$, and 7.13 lead to

$$
\begin{aligned}
\frac{d}{d t} \tilde{G}^{\text {odd }}(t, z) & =\sum_{l=1}^{\infty} \frac{1}{z^{l+1}} \frac{d}{d t} c_{2 l+1}(t)=\sum_{l=0}^{\infty} \frac{1}{z^{l+2}} \frac{d}{d t} c_{2 l+3}(t) \\
& =\sum_{l=0}^{\infty} \frac{1}{z^{l+2}}\left(2(l+1) \nu_{0} c_{2 l+1}(t)+4 \sum_{h=0}^{l}(l+1-h) c_{2 h}(t) c_{2 l+1-2 h}(t)\right) \\
& =-2 \nu_{0} \tilde{G}_{z}^{o d d}(t, z)-4 \tilde{G}^{\text {even }}(t, z) \tilde{G}_{z}^{\text {odd }}(t, z) .
\end{aligned}
$$

Please notice that we here interchanged derivatives and summations several times. This can be made precise by the same methods as in the proof of Proposition 2.9 by studying the corresponding approximating PDEs for the Stieltjes transforms of the measures $\mu_{N, t, \nu(N)}$.

Finally, the equations in (7.17) follow from that in 7.16 by an easy computation.

We now analyze the PDEs 7.17 with the corresponding initial data for $t=0$. Clearly, the quasilinear PDE for $G^{\text {even }}$ has to be solved first which then leads to the even parts $\mu_{t, \text { even }}$ of our probability measures $\mu_{t}$. On the other hand, the remark after Lemma 7.1 implies that the $\mu_{t, \text { even }}$ can be determined via the results in Section 4. More precisely, this remark and Theorem 4.8 imply:

Corollary 7.4. For $t \geq 0$, the even parts $\mu_{t, \text { even }}$ of the probability measures $\mu_{t}$ of Proposition 7.2 are the unique even probability measures on $\mathbb{R}$ whose pushforwards under the mapping $x \mapsto x^{2} / 2$ are given by $\mu_{M P, \nu_{0}, t} \boxplus\left(\mu_{s c, 2 \sqrt{t}} \boxplus \mu_{\text {even }}\right)^{2}$. Hence,

$$
\mu_{t, \text { even }}=\left(\sqrt{\mu_{M P, \nu_{0}, 2 t} \boxplus\left(\mu_{s c, 2 \sqrt{2 t}} \boxplus \mu_{\text {even }}\right)^{2}}\right)_{\text {even }} \quad(t \geq 0) .
$$


Therefore, if the initial measure $\mu_{0}=\mu$ is even, then the associated linear PDE for $G^{o d d}$ in 7.17 has the solution $G^{o d d}=0$, i.e., the $\mu_{t}$ of Proposition 7.2 are given by the measures in Eq. 7.20.

We now study the case where the initial measure $\mu_{0}=\mu$ is not even. We here have to solve the linear PDE of first order in 7.17 for $G^{\text {odd }}$ by using $G^{\text {even }}$ from Corollary 7.4. This can be carried out by classical methods on PDEs; see e.g. Section 1.2 of $[\mathrm{St}$. However, we can solve the second PDE (7.17) directly:

Lemma 7.5. Let $\mu \in M^{1}(\mathbb{R})$ be a probability measure for which the moment condition (2.13) holds. Let $D \subset H$ be some non-empty open domain such that there is some (analytical) function $K$ with

$$
K\left[G_{\mu_{\text {even }}}(z) \cdot\left(G_{\mu_{\text {even }}}(z)+\nu_{0} G_{\delta_{0}}(z)\right)\right]=z \quad(z \in D) .
$$

Then, with the measures $\mu_{t, \text { even }}$ from Eq. (7.20),

$$
G(t, z):=G_{\mu_{\text {odd }}}\left[K\left(G_{\mu_{t, \text { even }}}(z) \cdot\left(G_{\mu_{t, \text { even }}}(z)+\nu_{0} G_{\delta_{0}}(z)\right)\right)\right]+G_{\mu_{t, \text { even }}}(z)
$$

is the solution of (7.17) with the initial condition $G(0, z)=G_{\mu}(z)$ for $z \in D$.

In particular, for $\nu_{0}=0$ this solution simplifies to

$$
G(t, z)=G_{\mu}\left(G_{\mu_{\text {even }}}^{-1}\left(G_{\mu_{s c, 2 \sqrt{2 t}} \boxplus \mu_{\text {even }}}(z)\right)\right) .
$$

Proof. We first notice that the Stieltjes transforms $G_{\mu}, G_{\mu_{\text {even }}}$ are analytic on $\mathbb{C} \backslash \mathbb{R}$ where, by $7.15, G_{\mu_{\text {even }}}(w)=\frac{1}{w}+\sum_{l=1}^{\infty} \frac{c_{2 l}(0)}{w^{l+1}}$ for $|w|$ sufficiently large. This implies that $G_{\mu_{\text {even }}} \cdot\left(G_{\mu_{\text {even }}}+\nu_{0} G_{\delta_{0}}\right)$ is not constant on $H$ which ensures that there are plenty of non-empty open domains $D \subset H$ where this function admits some analytical inverse function $K$.

We now analyze the right hand side of $\sqrt{7.21}$. As $(z, t) \mapsto G_{\mu_{\text {, even }}}(z)$ solves the first PDE in (7.17) by Corollary 7.4, we only have to establish that the first summand of (7.21) solves the second PDE in (7.17), and that the initial value is correct. The first summand in (7.21) has the form

$$
G^{\text {odd }}(z, t):=f\left(G^{\text {even }}(z, t) \cdot\left(G^{\text {even }}(z, t)+\frac{\nu_{0}}{z}\right)\right) .
$$

Hence,

$$
G_{t}^{\text {odd }}(z, t)=f^{\prime}\left(G^{\text {even }}(z, t) \cdot\left(G^{\text {even }}(z, t)+\frac{\nu_{0}}{z}\right)\right) \cdot G_{t}^{\text {even }}(z, t)\left(2 G^{\text {even }}(z, t)+\frac{\nu_{0}}{z}\right)
$$

and

$$
\begin{aligned}
G_{z}^{\text {odd }}(z, t)= & f^{\prime}\left(G^{\text {even }}(z, t) \cdot\left(G^{\text {even }}(z, t)+\frac{\nu_{0}}{z}\right)\right) \cdot \\
& \cdot\left[2 G^{\text {even }}(z, t) G_{z}^{\text {even }}(z, t)+G_{z}^{\text {even }}(z, t) \cdot \frac{\nu_{0}}{z}-\frac{\nu_{0}}{z^{2}} G^{\text {even }}(z, t)\right] .
\end{aligned}
$$

These derivatives yield immediately that $G^{\text {odd }}$ satisfies the second PDE in (7.17). Moreover, a simple calculation shows that the corresponding initial value is correct.

Let us consider the special case $\nu_{0}=0$. Here, $G_{\mu_{t, \text { even }}}=G_{\mu_{s c, 2 \sqrt{2 t}} \boxplus \mu_{\text {even }}}$ and $K=\left(G_{\mu_{\text {even }}}^{2}\right)^{-1}$. This reduces the function in 7.21 to that in 7.22 .

We now discuss an example with $\nu_{0}=0$ where the initial distribution $\mu$ has a semicircle law as initial even part.

Example 7.6. Let the starting distribution $\mu$ be the quartercircle distribution on $[0,2]$ with Lebesgue density $\frac{1}{\pi} \sqrt{4-x^{2}} \mathbf{1}_{[0,2]}$. Then

$$
\mu_{\text {even }}=\mu_{s c, 2} \quad \text { and } \quad \mu_{s c, 2 \sqrt{2 t}} \boxplus \mu_{\text {even }}=\mu_{s c, 2 \sqrt{2 t+1}} .
$$


Moreover,

$$
G_{\mu_{s c, 2}}(z)=\frac{1}{2}\left(z-\sqrt{z^{2}-4}\right) \quad \text { and } \quad G_{\mu_{s c, 2}}^{-1}(z)=z+1 / z
$$

(see e.g. page 305 of $[\mathrm{AGZ}$ ) and thus

$$
\begin{aligned}
w(z):=G_{\mu_{s c, 2 \sqrt{2 t+1}}}(z) & =\frac{1}{\sqrt{2 t+1}} G_{\mu_{s c, 2}}\left(\frac{z}{\sqrt{2 t+1}}\right) \\
& =\frac{1}{2(2 t+1)}\left(z-\sqrt{z^{2}-4(2 t+1)}\right) .
\end{aligned}
$$

Now consider the probability measures $\mu_{t}$ for $t \geq 0$ whose even parts are the semicircle laws $\mu_{s c, 2 \sqrt{2 t+1}}$. The $\mu_{t}$ are supported on $[-2 \sqrt{2 t+1},+2 \sqrt{2 t+1}]$ and admit densities $f_{t}$. We recapitulate from Theorem 2.4.3 of AGZ that for $x \in$ ]$-2 \sqrt{2 t+1},+2 \sqrt{2 t+1}[$

$$
f_{t}(x)=\frac{-1}{\pi} \lim _{\epsilon \downarrow 0} \Im G(t, x+i \epsilon)
$$

where we can determine $G(t, x+i \epsilon)$ by Lemma 7.5. Moreover, as the even parts of the $\mu_{t}$ are already known, we can restrict our attention to $\left.x \in\right] 0,2 \sqrt{2 t+1}[$.

For $z:=x+i \epsilon$ with $\epsilon>0$ we then see that

$$
\lim _{\epsilon \downarrow 0} w(x+i \epsilon)=\frac{1}{2(2 t+1)}\left(x-i \sqrt{4(2 t+1)-x^{2}}\right)=w(x)=: w
$$

is contained in the closed fourth quadrant with $|w|=1 / \sqrt{2 t+1} \leq 1$. In particular, $1 / w$ is in the closed first quadrant with $|1 / w| \geq|w|$, and $1 / w+w$ and $1 / w-w$ are also in the closed first quadrant.

We next determine

$$
G_{\mu}(u)=\frac{1}{\pi} \int_{0}^{2} \frac{\sqrt{4-x^{2}}}{u-x} d x .
$$

As e.g. by WOLFRAM Alpha

$$
\begin{aligned}
\int \frac{\sqrt{4-x^{2}}}{u-x} d x= & -\sqrt{4-x^{2}}+u \arcsin (x / 2) \\
& +\sqrt{4-u^{2}}\left(\ln \left(\sqrt{\left(4-x^{2}\right)\left(4-u^{2}\right)}-x u+4\right)-\ln (u-x)\right)+c
\end{aligned}
$$

we obtain

$$
\begin{aligned}
G_{\mu}(u) & =\frac{2}{\pi}+\frac{u}{2}+\frac{\sqrt{4-u^{2}}}{\pi}\left(\ln (2-u)-\ln (u-2)-\ln \left(\sqrt{4-u^{2}}+2\right)+\ln u\right) \\
& =\frac{2}{\pi}+\frac{u}{2} \pm \sqrt{u^{2}-4}-\frac{\sqrt{4-u^{2}}}{\pi}\left(\ln \left(\sqrt{4-u^{2}}+2\right)-\ln u\right) .
\end{aligned}
$$

For $w$ as above we now define $u:=1 / w+w$. We then have $\sqrt{u^{2}-4}= \pm(1 / w-w)$. An analysis of the correct branch of the square root for $u$ in the closed first quadrant and 7.21 now lead to

$$
\begin{aligned}
\lim _{\epsilon \downarrow 0} G(t, x+i \epsilon) & =G_{\mu}\left(G_{\mu_{\text {even }}}^{-1}\left(G_{\mu_{s c, 2 \sqrt{2 t}} \boxplus \mu_{\text {even }}}(x)\right)\right) \\
& =\frac{2}{\pi}+\frac{1 / w+w}{2}+(w-1 / w)-\frac{i}{\pi}(1 / w-w) \ln \left(\frac{\sqrt{4-u^{2}}+2}{u}\right) \\
& =\frac{2}{\pi}+\frac{1 / w+w}{2}+(w-1 / w)-\frac{i}{\pi}(1 / w-w) \ln \left(\frac{i(1 / w-w)+2}{1 / w+w}\right) .
\end{aligned}
$$


Moreover, as $1 / w=(2 t+1) \bar{w}$, we obtain from 7.26 that

$$
\Im\left(\frac{1 / w+w}{2}+(w-1 / w)\right)=-\frac{1+t / 2}{2 t+1} \sqrt{4(2 t+1)-x^{2}}
$$

and

Furthermore, as

$$
\Im(w-1 / w)=-\frac{t+1}{2 t+1} \sqrt{4(2 t+1)-x^{2}}
$$

$$
\begin{aligned}
\frac{i(1 / w-w)+2}{1 / w+w} & =\frac{i-i w^{2}+2 w}{1+w^{2}}=\frac{(-i)\left(w^{2}+2 i w-1\right)}{1+w^{2}}=\frac{(-i)(w+i)^{2}}{(w+i)(w-i)} \\
& =\frac{(-i)(w+i)}{w-i}=\frac{(-i)(w+i)(\bar{w}+i)}{(w-i)(\bar{w}+i)}=\frac{(-i)(w+i)(\bar{w}+i)}{|w-i|^{2}} \\
& =\frac{w+\bar{w}+i(1-w \bar{w})}{|w-i|^{2}}
\end{aligned}
$$

we obtain from 7.26 that

$$
\Im \ln \left(\frac{i(1 / w-w)+2}{1 / w+w}\right)=\arctan \left(\frac{1-w \bar{w}}{w+\bar{w}}\right)=\arctan (2 t / x) .
$$

Finally, $\Re(w-1 / w)=-\frac{t x}{2 t+1}$ and

$$
\Re \ln \left(\frac{i(1 / w-w)+2}{1 / w+w}\right)=\Re \ln \left(\frac{w+i}{w-i}\right)=\frac{1}{2} \ln \left(\frac{2(t+1)-\sqrt{4(2 t+1)-x^{2}}}{2(t+1)+\sqrt{4(2 t+1)-x^{2}}}\right)
$$

In summary, we see from $\arctan y+\arctan (1 / y)=\pi / 2$ for $y>0$ that for $x \in$ ] $0,2 \sqrt{2 t+1}[$

$$
\begin{aligned}
f_{t}(x)= & \frac{-1}{\pi} \lim _{\epsilon \downarrow 0} \Im G(t, x+i \epsilon) \\
= & \frac{1}{(2 t+1) \pi}\left(\frac{1}{2}+\frac{t+1}{\pi} \arctan \frac{x}{2 t}\right) \sqrt{4(2 t+1)-x^{2}} \\
& -\frac{1}{\pi^{2}} \frac{t x}{2(2 t+1)} \ln \left(\frac{2(t+1)+\sqrt{4(2 t+1)-x^{2}}}{2(t+1)-\sqrt{4(2 t+1)-x^{2}}}\right) .
\end{aligned}
$$

Moreover, as the even part of $\mu_{t}$ is the semicircle law $\mu_{s c, 2 \sqrt{2 t+1}}$, Eq. 7.29 remains also valid for $x \in]-2 \sqrt{2 t+1}, 0[$.

In order to understand densities $f_{t}$ better, we define the rescaled densities

$$
\tilde{f}_{t}(x)=\sqrt{2 t+1} f(x \sqrt{2 t+1})
$$

of probability measures on $[-2,2]$. We then have for $x \in[-2,2]$,

$$
\begin{aligned}
\tilde{f}_{t}(x)= & \frac{1}{\pi}\left(\frac{1}{2}+\frac{t+1}{\pi} \arctan (\sqrt{2 t+1} x /(2 t))\right) \sqrt{4-x^{2}} \\
& -\frac{1}{\pi^{2}} \frac{t x}{2} \ln \left(\frac{1+\frac{\sqrt{2 t+1}}{2(t+1)} \sqrt{4-x^{2}}}{1-\frac{\sqrt{2 t+1}}{2(t+1)} \sqrt{4-x^{2}}}\right) .
\end{aligned}
$$

A Taylor expansion of the arctan- and ln-term now yields that

$$
\tilde{f}_{t}(x)=\frac{\sqrt{4-x^{2}}}{2 \pi}+\frac{2 \sqrt{2} \cdot x \sqrt{4-x^{2}}}{3 \pi^{2}} \cdot O\left(\frac{1}{\sqrt{t}}\right)+O(1 / t)
$$


for $t \rightarrow \infty$ where the term $O\left(\frac{1}{\sqrt{t}}\right)$ is independent of $x \in[-2,2]$ while the term $O(1 / t)$ may depend on $x$. In particular, for $t \rightarrow \infty, \tilde{f}_{t}$ tends to the density of the semicircle law $\mu_{s c, 2}$, i.e., the influence of the asymmetric starting measure vanishes of order $O\left(\frac{1}{\sqrt{t}}\right)$. Figure 1 illustrates the time-behaviour of $\tilde{f}_{t}(x)$. It is plotted for $t=0.1$ (bold black), $t=1$ (dashed), $t=10$ (dashed small), $t=100$ (dotted) together with the start $t=0$ and the limit $t \rightarrow \infty$ (both grey).

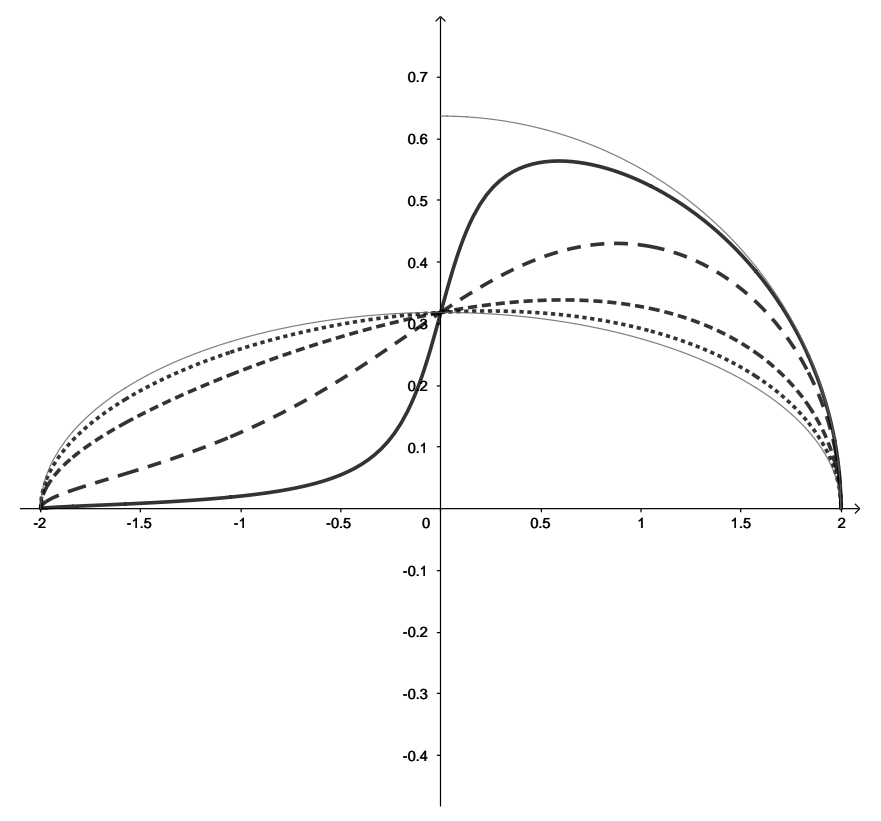

Figure 1. $\tilde{f}_{t}(x)$ for $t=0,0.1,1,10,100$ and $t=\infty$.

\section{Limit theorems for DunkL PRocesses of type B}

In this section we proceed to the next step and study the empirical distributions of normalized Dunkl processes $\left(\tilde{X}_{t, \nu, \beta}\right)_{t \geq 0}$ of type $B$ on $\mathbb{R}^{N}$ with the generators $\tilde{\mathcal{L}}_{\nu, \beta}=\frac{1}{2 \beta} \Delta+L_{\nu}$ with $L_{\nu}$ as in 6.4 ; ; see Example 6.2. On some informal level, the processes $\left(\tilde{X}_{t, \nu, \beta}\right)_{t \geq 0}$ converge for $\beta \rightarrow \infty$ to the frozen Dunkl processes. We assume that these processes start in deterministic points in $\mathbb{R}^{N}$ independent of $\beta$.

We denote the components of $X_{t, \nu, \beta}$ by $X_{t, \nu, \beta}^{j}$ for $j=1, \ldots, N$, and similar to Sections 3 and 5 we study the random normalized empirical measures

$$
\mu_{N, t, \nu, \beta}:=\frac{1}{N}\left(\delta_{\tilde{X}_{t, \nu, \beta}^{1} / \sqrt{N}}+\ldots+\delta_{\tilde{X}_{t, \nu, \beta}^{N} / \sqrt{N}}\right) \in M^{1}(\mathbb{R})
$$

of the processes $\left(\tilde{X}_{t, \nu, \beta}\right)_{t \geq 0}$ for $N \rightarrow \infty$. We claim that the measures $\mu_{N, t, \nu, \beta}$ converge to the same limit as the normalized empirical measures of the expectations of the frozen Dunkl process of the previous section. For this we study the moments

$$
S_{N, l, \nu, \beta}(t):=\frac{1}{N^{l / 2+1}}\left(\left(\tilde{X}_{t, \nu, \beta}^{1}\right)^{l}+\ldots+\left(\tilde{X}_{t, \nu, \beta}^{N}\right)^{l}\right) \quad(l \geq 0) .
$$


By the construction of the processes $\left(\tilde{X}_{t, \nu, \beta}\right)_{t \geq 0}$, the even moments $S_{N, 2 l, \nu, \beta}(t)$ are closely related to corresponding moments of the Bessel processes of type B, and for them we can proceed as in Section 5 . The odd moments however are different due to the additional jump components. We proceed as in Section 3 with a Lemma concerning the symmetric monomials $m_{\lambda}$ and refer to the notation there.

Lemma 8.1. Let $\left(x_{N}\right)_{N \geq 1}:=\left(x_{N, n}\right)_{N \geq 1,1 \leq n \leq N} \subset \mathbb{R}$ be a family of starting numbers with $x_{N, n-1} \geq x_{N, n}$ for $2 \leq n \leq N$, for which

$$
\lim _{N \rightarrow \infty} \frac{m_{\lambda}\left(x_{N}\right)}{N ! \cdot N^{|\lambda| / 2}}<\infty
$$

exists for all $\lambda \in \mathcal{P}$.

Let $\beta \in[1 / 2, \infty], \nu>0$, and $\left(\tilde{X}_{t, \nu, \beta}\right)_{t \geq 0}$ the renormalized Dunkl processes with start in $\left(x_{N, 1}, \ldots, x_{N, n}\right)$. Then, for all $\lambda \in \mathcal{P}$, the limits

$$
\lim _{N \rightarrow \infty} \frac{E\left(m_{\lambda}\left(\tilde{X}_{t, \nu, \beta}\right)\right)}{N ! \cdot N^{|\lambda| / 2}}
$$

exist locally uniformly in $t$ and are independent from $\beta$.

Proof. We prove this statement by induction on $|\lambda|$. For $\lambda=0$ we have $m_{\lambda}(x)=N$ ! and thus the claim. For $\lambda=(1,0, \ldots, 0)$ we have $m_{\lambda}(x)=(N-1) ! \cdot\left(x_{1}+\ldots+x_{N}\right)$. Thus, by Itô's formula for Dunkl processes in Corollary 3.6 of [CGY]

$$
\sum_{i=1}^{N} \tilde{X}_{t, \nu, \beta}^{i}=\sum_{i=1}^{N} x_{N, i}+\frac{1}{\sqrt{\beta}} \sum_{i=1}^{N} B_{t}^{i}+\sqrt{2 \nu} \sum_{i=1}^{N} M_{t}^{i}+2 \sum_{i=1}^{N} \sum_{j \neq i} M_{t}^{i j-},
$$

where, by Eq. (50) of [CGY], the jump components of the normalized Dunkl process $M^{i}$ and $M^{i j^{-}}$associated to the different roots are given by

$$
\begin{aligned}
M_{t}^{i} & =\sum_{s \leq t} \frac{-\sqrt{2} \tilde{X}_{s-, \nu, \beta}^{i}}{\sqrt{\nu}} \mathbf{1}_{\left(-\tilde{X}_{s, \nu, \beta}^{i} \neq \tilde{X}_{s-, \nu, \beta}^{i}\right)}+\int_{0}^{t} \frac{\sqrt{\nu}}{\sqrt{2} \tilde{X}_{s-, \nu, \beta}^{i}} d s \\
M_{t}^{i j^{-}} & =\sum_{s \leq t}-\left(\tilde{X}_{s-, \nu, \beta}^{i}+\tilde{X}_{s-, \nu, \beta}^{j}\right) \mathbf{1}_{\left(-\tilde{X}_{s, \nu, \beta}^{i} \neq \tilde{X}_{s-, \nu, \beta}^{j}\right)}+\int_{0}^{t} \frac{1}{\tilde{X}_{s-, \nu, \beta}^{i}+\tilde{X}_{s-, \nu, \beta}^{j}} d s .
\end{aligned}
$$

Notice that in the RHS of 8.3 the additional sum $S:=2 \sum_{i=1}^{N} \sum_{j \neq i} M_{t}^{i j}$ with

$$
M_{t}^{i j}=\sum_{s \leq t}-\left(\tilde{X}_{s-, \nu, \beta}^{i}-\tilde{X}_{s-, \nu, \beta}^{j}\right) \mathbf{1}_{\left(\tilde{X}_{s, \nu, \beta}^{i} \neq \tilde{X}_{s-, \nu, \beta}^{j}\right)}+\int_{0}^{t} \frac{1}{\tilde{X}_{s-, \nu, \beta}^{i}-\tilde{X}_{s-, \nu, \beta}^{j}} d s
$$

appears for which $S=0$ holds. As the $M_{t}^{i}, M_{t}^{i j^{-}}$are martingales by [CGY], the claim follows for $|\lambda|=1$.

Now let $\lambda \in \mathcal{P}$ with $|\lambda| \geq 2$; assume that the statement is already shown for partitions with weight at most $|\lambda|-1$. Itô's formula in Corollary 3.6 of [CGY] yields

$m_{\lambda}\left(\tilde{X}_{t, \nu, \beta}\right)=m_{\lambda}\left(x_{N}\right)+\frac{1}{\sqrt{\beta}} \sum_{i=1}^{N} \int_{0}^{t} \frac{d m_{\lambda}}{d x_{i}}\left(\tilde{X}_{s, \nu, \beta}\right) d B_{s}^{i}+\mathcal{M}_{t}+\int_{0}^{t}\left(\tilde{\mathcal{L}}_{\nu, \beta} m_{\lambda}\right)\left(\tilde{X}_{s, \nu, \beta}\right) d s$ 
with

$$
\begin{aligned}
& \mathcal{M}_{t}:=\sqrt{\nu} \sum_{\pi \in S_{N}} \sum_{i=1}^{N} \int_{0}^{t} \frac{\left(\left(\tilde{X}_{s-, \nu, \beta}^{i}\right)^{\lambda_{\pi(i)}}-\left(-\tilde{X}_{s-, \nu, \beta}^{i}\right)^{\lambda_{\pi(i)}}\right)\left(\tilde{X}_{s-, \nu, \beta}^{\pi(\lambda)}\right)_{i}}{\sqrt{2} \tilde{X}_{s-, \nu, \beta}^{i}} d M_{s}^{i} \\
& +\sum_{\pi \in S_{N}} \sum_{i, j: j \neq i} \int_{0}^{t} \frac{\left(\tilde{X}_{s-, \nu, \beta}^{i}\right)^{\lambda_{\pi(i)}}\left(\tilde{X}_{s-, \nu, \beta}^{j}\right)^{\lambda_{\pi(j)}}-\left(-\tilde{X}_{s-, \nu, \beta}^{i}\right)^{\lambda_{\pi(j)}}\left(-\tilde{X}_{s-, \nu, \beta}^{j}\right)^{\lambda_{\pi(i)}}}{\tilde{X}_{s-, \nu, \beta}^{i}+\tilde{X}_{s-, \nu, \beta}^{j}} \\
& \cdot\left(\tilde{X}_{s-, \nu, \beta}^{\pi(\lambda)}\right)_{i, j} d M_{s}^{i j^{-}} \\
& +\sum_{\pi \in S_{N}} \sum_{i, j: j \neq i} \int_{0}^{t} \frac{\left(\tilde{X}_{s-, \nu, \beta}^{i}\right)^{\lambda_{\pi(i)}}\left(\tilde{X}_{s-, \nu, \beta}^{j}\right)^{\lambda_{\pi(j)}}-\left(\tilde{X}_{s-, \nu, \beta}^{i}\right)^{\lambda_{\pi(j)}}\left(\tilde{X}_{s-, \nu, \beta}^{j}\right)^{\lambda_{\pi(i)}}}{\tilde{X}_{s-, \nu, \beta}^{i}-\tilde{X}_{s-, \nu, \beta}^{j}} \\
& \cdot\left(\tilde{X}_{s-, \nu, \beta}^{\pi(\lambda)}\right)_{i, j} d M_{s}^{i j}
\end{aligned}
$$

where $\left(x^{\pi(\lambda)}\right)_{i}$ and $\left(x^{\pi(\lambda)}\right)_{i, j}$ denote the multivariate products as in Section 3 where the factors involving $x_{i}$ or in addition $x_{j}$ are omitted respectively.

The diffusion parts $\frac{1}{\sqrt{\beta}} \sum_{i=1}^{N} \int_{0}^{t} \frac{d m_{\lambda}}{d x_{i}}\left(\tilde{X}_{s, \nu, \beta}\right) d B_{s}^{i}$ in 8.4 are martingales by the same arguments as in the proof of Lemma 3.1, taking into account that the sum of the squared components is again a one-dimensional Bessel process as all contributions from the jump components vanish. This yields

$$
E\left(\sum_{i=1}^{N} \int_{0}^{t} \frac{d m_{\lambda}}{d x_{i}}\left(\tilde{X}_{s, \nu, \beta}\right) d B_{s}^{i}\right)=0 \quad(t \geq 0) .
$$

Moreover, the integrals w.r.t. $\left(M_{t}^{i}\right)_{t},\left(M_{t}^{i j^{-}}\right)_{t},\left(M_{t}^{i j}\right)_{t}$ are also martingales and hence their expectations equal to zero. This follows easily from the representation of these martingales $\left(M_{t}^{i}\right)_{t},\left(M_{t}^{i j^{-}}\right)_{t},\left(M_{t}^{i j}\right)_{t}$ as compensated sums of jumps as on p. 125 of CGY; for instance, for $\left(M_{t}^{i}\right)_{t}$ we have

$$
\begin{aligned}
\sum_{\pi \in S_{N}} & \sum_{i=1}^{N} \int_{0}^{t} \frac{\left(\left(\tilde{X}_{s-, \nu, \beta}^{i}\right)^{\lambda_{\pi(i)}}-\left(-\tilde{X}_{s-, \nu, \beta}^{i}\right)^{\lambda_{\pi(i)}}\right)\left(\tilde{X}_{s-, \nu, \beta}^{\pi(\lambda)}\right)_{i}}{\sqrt{2} \tilde{X}_{s-, \nu, \beta}^{i}} \sqrt{\nu} d M_{s}^{i} \\
= & -\sum_{\pi \in S_{N}} \sum_{i=1}^{N} \sum_{s \leq t}\left(\left(\tilde{X}_{s-, \nu, \beta}^{i}\right)^{\lambda_{\pi(i)}}-\left(-\tilde{X}_{s-, \nu, \beta}^{i}\right)^{\lambda_{\pi(i)}}\right)\left(\tilde{X}_{s-, \nu, \beta}^{\pi(\lambda)}\right)_{i} \mathbf{1}_{\left(-\tilde{X}_{s, \nu, \beta}^{i} \neq \tilde{X}_{s-, \nu, \beta}^{i}\right)} \\
& +\nu \int_{0}^{t} \sum_{\pi \in S_{N}} \sum_{i=1}^{N}\left(\left(\tilde{X}_{s-, \nu, \beta}^{i}\right)^{\lambda_{\pi(i)}}-\left(-\tilde{X}_{s-, \nu, \beta}^{i}\right)^{\lambda_{\pi(i)}}\right)\left(\tilde{X}_{s-, \nu, \beta}^{\pi(\lambda)}\right)_{i} d s
\end{aligned}
$$

We finally turn to the drift term of the RHS in in 8.4. We there observe that by the theory of Dunkl operators (see e.g. [DX]) $\tilde{\mathcal{L}}_{\nu, \beta} m_{\lambda}$ is a homogeneous polynomial of the order $|\lambda|-2$. Moreover, by the definition of $\tilde{\mathcal{L}}_{\nu, \beta}$ in Section 6 , it can be easily checked that it also symmetric and that it has the form $\frac{1}{2 \beta} Q_{\lambda}+R_{\lambda}$ with

$$
Q_{\lambda}(x)=\sum_{\pi \in S_{N}} \sum_{i=1}^{N} \lambda_{\pi(i)}\left(\lambda_{\pi(i)}-1\right)\left(x_{i}\right)^{\lambda_{\pi(i)}-2}\left(x^{\pi(\lambda)}\right)_{i}
$$

and with some symmetric homogeneous polynomial $R_{\lambda}$ of order $|\lambda|-2$ which only depends on $\nu$, but not on $\beta$. The methods of the proof of (3.8) show that $Q_{\lambda}$ is a linear combination of the $m_{\tilde{\lambda}}$ with $|\tilde{\lambda}|=|\lambda|-2$ with coefficients independent of $N \geq L(\lambda)$. Moreover, as in the proof of Lemma 3.1. $R_{\lambda}$ is a linear combination of 
the $m_{\tilde{\lambda}}$ with $|\tilde{\lambda}|=|\lambda|-2$ with coefficients $c_{\tilde{\lambda}}$ such that the terms $c_{\tilde{\lambda}} / N$ converge to some limits for $N \rightarrow \infty$. As in the proof of Lemma 3.1, these assertions together with the induction assumption now lead to claim for $\lambda$.

Remark 8.2. The proof of Lemma 8.1 shows that for fixed $\beta$ and $\lambda$, the limit in Lemma 8.1 has order $O(1 / N)$.

Lemma 8.1 has the following application to the moments $S_{N, l}(t)$ :

Corollary 8.3. Let $\left(x_{N, n}\right)_{N \geq 1,1 \leq n \leq N} \subset \mathbb{R}$ be starting numbers with $x_{N, n-1} \geq x_{N, n}$ for $2 \leq n \leq N$, for which the convergence condition in Lemma 7.1 holds. Let $\beta \in[1 / 2, \infty], \nu>0$, and let for $N \geq 2,\left(\tilde{X}_{t, \nu, \beta}\right)_{t \geq 0}$ the renormalized Dunkl processes starting in $\left(x_{N, 1}, \ldots, x_{N, n}\right)$. Then, for $l \in \mathbb{N}_{0}$ and $c_{l}(t)$ from Lemma 7.1.

$$
E\left(S_{N, l}(t)\right) \rightarrow c_{l}(t) \quad \text { for } \quad N \rightarrow \infty
$$

Proof. This follows from Lemma 8.1 analogous to the proof of Corollary 3.3

Corollary 8.3 can be extended to an a.s. result:

Theorem 8.4. Consider the Dunkl processes $\left(\tilde{X}_{t, \nu, \beta}\right)_{t \geq 0}$ with $\beta \geq 1 / 2, \nu>0$ and with starting sequences $\left(x_{N, i}\right)_{i \geq 1} \subset \mathbb{R}$ as before such that for $l \geq 0$,

$$
c_{l}(0):=\lim _{N \rightarrow \infty} S_{N, l, \nu, \beta}(0)=\lim _{n \rightarrow \infty} \frac{1}{N^{l / 2+1}}\left(x_{N, 1}^{l}+\ldots+x_{N, N}^{l}\right)<\infty
$$

exists. Let $\nu=\nu(N)$ with $\nu_{0}:=\lim _{N \rightarrow \infty} \nu(N) / N \geq 0$. Then, for $l \in \mathbb{N}_{0}$,

$$
c_{l}(t):=\lim _{N \rightarrow \infty} S_{N, l, \nu, \beta}(t)
$$

exists a.s. locally uniformly in $t \in\left[0, \infty\left[\right.\right.$. Furthermore, the $c_{l}(t)$ satisfy the recurrence relation from Lemma 7.1, i.e., $c_{0}(t)=1, c_{1}(t)=c_{1}(0)$, and for $l \geq 1$,

$$
\begin{aligned}
c_{2 l}(t) & =c_{2 l}(0)+2 l \int_{0}^{t}\left(\nu_{0} c_{2 l-2}(s)+\sum_{h=0}^{l-1} c_{2 h}(s) c_{2 l-2 h-2}(s)\right) d s \\
c_{2 l+1}(t) & =c_{2 l+1}(0)+\int_{0}^{t}\left(2 l \nu_{0} c_{2 l-1}(s)+4 \sum_{h=0}^{l-1}(l-h) c_{2 h}(s) c_{2 l-2 h-1}(s)\right) d s .
\end{aligned}
$$

Proof. Again, by Itô's formula for Dunkl processes in Corollary 3.6 of [CGY], we obtain for $l \geq 1$

$$
\begin{aligned}
& \sum_{i=1}^{N}\left(\tilde{X}_{t, \nu, \beta}^{i}\right)^{l}=\sum_{i=1}^{N} x_{i}^{l}+D_{l}(t)+\frac{l}{\sqrt{\beta}} \sum_{i=1}^{N} \int_{0}^{t}\left(\tilde{X}_{s, \nu, \beta}^{i}\right)^{l-1} d B_{s}^{i} \\
& \quad+\sum_{i=1}^{N} \int_{0}^{t} \frac{\left(\tilde{X}_{s-, \nu, \beta}^{i}\right)^{l}-\left(-\tilde{X}_{s-, \nu, \beta}^{i}\right)^{l}}{\sqrt{2} \tilde{X}_{s-, \nu, \beta}^{i}} \sqrt{\nu} d M_{s}^{i} \\
& \quad+\sum_{i=1}^{N} \sum_{j \neq i} \int_{0}^{t} \frac{\left(\tilde{X}_{s-, \nu, \beta}^{i}\right)^{l}-\left(-\tilde{X}_{s-, \nu, \beta}^{j}\right)^{l}+\left(\tilde{X}_{s-, \nu, \beta}^{j}\right)^{l}-\left(-\tilde{X}_{s-, \nu, \beta}^{i}\right)^{l}}{\tilde{X}_{s-, \nu, \beta}^{i}+\tilde{X}_{s-, \nu, \beta}^{j}} d M_{s}^{i j^{-}}
\end{aligned}
$$


with the drift term

$$
\begin{aligned}
D_{l}(t) & :=\int_{0}^{t} l \sum_{i=1}^{N} \sum_{j \neq i} \frac{2\left(\tilde{X}_{s, \nu, \beta}^{i}\right)^{l}}{\left(\tilde{X}_{s, \nu, \beta}^{i}\right)^{2}-\left(\tilde{X}_{s, \nu, \beta}^{j}\right)^{2}} d s \\
& +\sum_{i=1}^{N} \int_{0}^{t}\left(l \nu+\frac{l(l-1)}{2 \beta}\right)\left(\tilde{X}_{s, \nu, \beta}^{i}\right)^{l-2} d s \\
& +\frac{\nu}{2} \int_{0}^{t} \sum_{i=1}^{N} \frac{\left(-\tilde{X}_{s-, \nu, \beta}^{i}-\left(\tilde{X}_{s-, \nu, \beta}^{i}\right)^{l}\right.}{\left(\tilde{X}_{s-, \nu, \beta}^{i}\right)^{2}} d s \\
& +\frac{1}{2} \int_{0}^{t} \sum_{i=1}^{N} \sum_{j \neq i} \frac{\left(-\tilde{X}_{s-, \nu, \beta}^{i}\right)^{l}+\left(-\tilde{X}_{s-, \nu, \beta}^{j}\right)^{l}-\left(\tilde{X}_{s-, \nu, \beta}^{j}\right)^{l}-\left(\tilde{X}_{s-, \nu, \beta}^{i}\right)^{l}}{\left(\tilde{X}_{s-, \nu, \beta}^{i}+\tilde{X}_{s-, \nu, \beta}^{j}\right)^{2}} d s .
\end{aligned}
$$

Please notice that here the sums of the integrals w.r.t. the $M^{i j}$ are zero and thus omitted. As in the proof of Lemma 8.1 the integrals with respect to $B^{i}, M^{i}, M^{i j-}$ in the RHS of (8.6) are martingales. For simplicity we denote them by $A^{1, l}, A^{2, l}, A^{3, l}$ respectively. We also notice that the covariations between the jump processes associated to different roots are zero by Eq. (49) in CGY.

As for the even moments of order $2 l$ all terms associated with the jump component of the Dunkl process vanish, we are left with the terms of a Bessel process of type B, and the claim follows by the results of Section 5 .

Hence it remains to prove the claim for the odd moments. Here we proceed similar to the proof of Theorem 3.4 where we now apply the Burkholder-DavisGundy inequality with exponent four in order to get a sufficiently fast convergence of the bound leading to a.s. convergence in the end. In fact, 8.6 together with the Markov inequality, Burkholder-Davis-Gundy inequality with exponent 4 and the inequality $(a+b+c)^{2} \leq 3\left(a^{2}+b^{2}+c^{2}\right)$ for $a, b, c \in \mathbb{R}$ show that for all $l \in \mathbb{N}_{0}$, $\epsilon>0$ and $T>0$ and some universal constant $c>0$,

$$
\begin{aligned}
\sup _{s \leq T} P(\mid & \left.\frac{1}{N^{\frac{2 l+1}{2}}+1}\left(\sum_{i=1}^{N}\left(\tilde{X}_{s, \nu, \beta}^{i}\right)^{2 l+1}-\sum_{i=1}^{N} x_{N, i}^{2 l+1}-D_{t}^{2 l+1}\right) \mid>\epsilon\right) \\
& \leq \frac{1}{\epsilon^{4}} E\left(\sup _{s \leq T}\left(\frac{1}{N^{\frac{2 l+1}{2}+1}}\left(A^{1,2 l+1}+A^{2,2 l+1}+A^{3,2 l+1}\right)\right)^{4}\right) \\
& \leq \frac{c}{\epsilon^{4}} \frac{1}{N^{4 l+6}} E\left(\left(\sum_{i=1}^{3}\left[A^{i, 2 l+1}, A^{i, 2 l+1}\right]_{T}\right)^{2}\right) \\
& \leq \frac{3 c}{\epsilon^{4}} \frac{1}{N^{2}} \sum_{i=1}^{3} E\left(\frac{1}{N^{4 l+4}}\left[A^{i, 2 l+1}, A^{i, 2 l+1}\right]_{T}^{2}\right) \\
& =: \frac{3 c}{\epsilon^{4}} \frac{1}{N^{2}} B_{N, T, 2 l+1} .
\end{aligned}
$$

We next prove

$$
\sum_{N=1}^{\infty} \frac{1}{N^{2}} B_{N, T, 2 l+1}<\infty
$$

For this, we show that $\lim _{N \rightarrow \infty} B_{N, T, 2 l+1}<\infty$, which holds if

$$
\lim _{N \rightarrow \infty} E\left(\frac{1}{N^{4 l+4}}\left[A^{i, 2 l+1}, A^{i, 2 l+1}\right]_{T}^{2}\right)<\infty \quad \text { for } \quad i=1,2,3 .
$$


We consider these expectations separately with the aid of Corollary 8.3 .

The Brownian martingale $A^{1,2 l+1}$ can be handled as in the proof of Theorem 3.4 ; in fact, Hölder's inequality and Corollary 8.3 yield that

$$
\begin{aligned}
E\left(\frac{1}{N^{4 l+4}}\left[A^{1,2 l+1}, A^{1,2 l+1}\right]_{T}^{2}\right) & =E\left(\frac{(2 l+1)^{2}}{\beta N^{4 l+4}}\left(\sum_{i=1}^{N} \int_{0}^{T}\left(\tilde{X}_{s, \nu, \beta}^{i}\right)^{4 l} d s\right)^{2}\right) \\
& \leq \frac{(2 l+1)^{2}}{\beta N^{4 l+4}} E\left(N \sum_{i=1}^{N}\left(\int_{0}^{T}\left(\tilde{X}_{s, \nu, \beta}^{i}\right)^{4 l} d s\right)^{2}\right) \\
& \leq \frac{T(2 l+1)^{2}}{\beta N^{2}} E\left(\sum_{i=1}^{N} \int_{0}^{T} \frac{\left(\tilde{X}_{s, \nu, \beta}^{i}\right)^{8 l}}{N^{4 l+1}} d s\right) \\
& =\frac{T(2 l+1)^{2}}{\beta N^{2}} \int_{0}^{T} E\left(S_{N, 4 l, \nu, \beta}(s)\right) d s \rightarrow 0
\end{aligned}
$$

for $N \rightarrow \infty$. For $A^{2,2 l+1}$ we use Eq. (48) from [CGY], Hölder's inequality, and Corollary 8.3 again and conclude that

$$
\begin{aligned}
E\left(\frac{1}{N^{4 l+4}}\left[A^{2,2 l+1}, A^{2,2 l+1}\right]_{T}^{2}\right) & =E\left(\frac{4 \nu^{2}}{N^{4 l+4}}\left(\sum_{i=1}^{N} \int_{0}^{T}\left(\tilde{X}_{s-, \nu, \beta}^{i}\right)^{4 l} d\left[M^{i}, M^{i}\right]_{s}\right)^{2}\right) \\
& \leq \frac{4 \nu^{2}}{N^{4 l+4}} E\left(N \sum_{i=1}^{N}\left(\int_{0}^{T}\left(\tilde{X}_{s-, \nu, \beta}^{i}\right)^{4 l} d s\right)^{2}\right) \\
& \leq 4 T \frac{\nu^{2}}{N^{2}} \sum_{i=1}^{N} E\left(\int_{0}^{T} \frac{\left(\tilde{X}_{s-, \nu, \beta}^{i}\right)^{8 l}}{N^{4 l+1}} d s\right)
\end{aligned}
$$

remains bounded for $N \rightarrow \infty$; notice here that $\nu / N$ tends to $\nu_{0}$. Moreover, using the polynom division as in $(7.7)$ and Hölder's inequality three times, we see from Lemma 8.1 that

$$
\begin{aligned}
E & \left.\frac{1}{N^{4 l+4}}\left[A^{3,2 l+1}, A^{3,2 l+1}\right]_{T}^{2}\right) \\
& =E\left(\frac{4}{N^{4 l+4}}\left(\sum_{i, j: j \neq i} \int_{0}^{T}\left(\sum_{h=0}^{2 l}(-1)^{h}\left(\tilde{X}_{s-, \nu, \beta}^{i}\right)^{h}\left(\tilde{X}_{s-, \nu, \beta}^{j}\right)^{2 l-h}\right)^{2} d\left[M^{i j^{-}}, M^{i j^{-}}\right]_{s}\right)^{2}\right) \\
& \leq \frac{4}{N^{4 l+4}} E\left(N(N-1) \sum_{i, j: j \neq i}\left(\int_{0}^{T}\left(\sum_{h=0}^{2 l}(-1)^{h}\left(\tilde{X}_{s-, \nu, \beta}^{i}\right)^{h}\left(\tilde{X}_{s-, \nu, \beta}^{j}\right)^{2 l-h}\right)^{2} d s\right)^{2}\right) \\
& \leq \frac{4 T}{N^{4 l+4}} E\left(N(N-1) \sum_{i, j: j \neq i} \int_{0}^{T}\left(\sum_{h=0}^{2 l}(-1)^{h}\left(\tilde{X}_{s-, \nu, \beta}^{i}\right)^{h}\left(\tilde{X}_{s-, \nu, \beta}^{j}\right)^{2 l-h}\right)^{4} d s\right) \\
& \leq 4 T(2 l+1)^{3} E\left(\frac{N(N-1)}{N^{2}} \sum_{i, j: j \neq i} \int_{0}^{T} \sum_{h=0}^{2 l} \frac{\left(\tilde{X}_{s-, \nu, \beta}^{i}\right)^{4 h}}{N^{2 h+1}} \frac{\left(\tilde{X}_{s-, \nu, \beta}^{j}\right)^{8 l-4 h}}{N^{4 l-2 h+1}} d s\right)
\end{aligned}
$$

also remains bounded for $N \rightarrow \infty$. This completes the proof of $(8.8)$.

Looking at the drift term $D^{2 l+1}$ of the RHS of 8.6 , we obtain the recurrence relation 7.13, where $\nu$ is replaced by $\nu+\frac{l-1}{2 \beta}$. The desired results now follow by the same arguments as in the proof of Theorem 3.4 using the results of Lemma 7.1 . as well as Theorem 4.8, 
As in Section 3, Theorem 8.4 leads to the following final limit theorem.

Theorem 8.5. Let $\mu \in M^{1}([0, \infty[)$ be a probability measure which satisfies the moment condition 2.13). Let $\left(x_{N, n}\right)_{N \geq 1,1 \leq n \leq N} \subset[0, \infty[$ such that the empirical measures

$$
\mu_{N, 0}:=\frac{1}{N}\left(\delta_{x_{N, 1} / \sqrt{N}}+\ldots \delta_{x_{N, N} / \sqrt{N}}\right)
$$

tend weakly to $\mu$ for $N \rightarrow \infty$. Consider the normalized Dunkl processes $\left(\tilde{X}_{t, \nu, \beta}\right)_{t \geq 0}$ of type $B$ with start in $\left(x_{N, 1}, \ldots, x_{N, N}\right)$ for $N \geq 2$. Then, for $t \geq 0$ and

$$
\lim _{N \rightarrow \infty} \frac{\nu(N)}{N}=\nu_{0} \geq 0
$$

the empirical measures

$$
\mu_{N, t}:=\frac{1}{N}\left(\delta_{\frac{\tilde{x}_{t, \nu, \beta}^{1},}{\sqrt{N}}}+\ldots+\delta_{\frac{\tilde{x}_{t, \nu, \beta}^{N}}{\sqrt{N}}}\right)
$$

tend weakly a.s. to the limiting measure whose Stieltjes transform satisfies the PDEs (7.17) with the corresponding initial condition.

\section{REFERENCES}

[A] N.I. Akhiezer, The Classical Moment Problems and Some Related Questions in Analysis. Engl. Translation, Hafner Publishing Co., New York, 1965.

[AGZ] G.W. Anderson, A. Guionnet, O. Zeitouni, An Introduction to Random Matrices. Cambridge University Press, 2010.

[AHV] S. Andraus, K. Hermann, M. Voit, Limit theorems and soft edge of freezing random matrix models via dual orthogonal polynomials. Preprint, arXiv:2009.01418.

[AKM1] S. Andraus, M. Katori, S. Miyashita, Interacting particles on the line and Dunkl intertwining operator of type A: Application to the freezing regime. J. Phys. A: Math. Theor. 45 (2012) 395201.

[AKM2] S. Andraus, M. Katori, S. Miyashita, Two limiting regimes of interacting Bessel processes. J. Phys. A: Math. Theor. 47 (2014) 235201.

[AV1] S. Andraus, M. Voit, Limit theorems for multivariate Bessel processes in the freezing regime. Stoch. Proc. Appl. 129 (2019), 4771-4790.

[AV2] S. Andraus, M. Voit, Central limit theorems for multivariate Bessel processes in the freezing regime II: the covariance matrices of the limit. J. Approx. Theory 246 (2019), 65-84.

[An] J.-P. Anker, An introduction to Dunkl theory and its analytic aspects. In: G. Filipuk, Y. Haraoka, S. Michalik. Analytic, Algebraic and Geometric Aspects of Differential Equations, Birkhäuser, Cham (Switzerland), pp.3-58, 2017.

[CG] T. Cabanal Duvillard, A. Guionnet, Large deviations upper bounds for the laws of matrixvalued processes and non-commutative entropies. Ann. Probab. 29 (2001), 1205-1261.

[CGY] O. Chybiryakov, L. Gallardo, M. Yor, Dunkl processes and their radial parts relative to a root system. In: P. Graczyk et al. (eds.), Harmonic and stochastic analysis of Dunkl processes, pp. 113-198. Hermann, Paris 2008.

[D] P. Deift, Orthogonal Polynomials and Random Matrices: A Riemann-Hilbert Approach. Amer. Math. Soc. 2000.

[DV] J.F. van Diejen, L. Vinet, Calogero-Sutherland-Moser Models. CRM Series in Mathematical Physics, Springer, Berlin, 2000.

[DE1] I. Dumitriu, A. Edelman, Matrix models for beta-ensembles. J. Math. Phys. 43 (2002), 5830-5847.

[DE2] I. Dumitriu, A. Edelman, Eigenvalues of Hermite and Laguerre ensembles: large beta asymptotics, Ann. Inst. Henri Poincare (B) 41 (2005), 1083-1099.

[DX] C.F. Dunkl, Y. Xu, Orthogonal Polynomials of Several Variables. Cambridge University Press, Cambridge, 2001.

[GY] L. Gallardo, M. Yor, Some remarkable properties of the Dunkl martingale. In: Seminaire de Probabilites XXXIX, pp. 337-356, dedicated to P.A. Meyer, vol. 1874, Lecture Notes in Mathematics, Springer, Berlin, 2006. 
[G] W. Gawronski, On the asymptotic distribution of the zeros of Hermite, Laguerre, and Jonquiere polynomials. J. Approx. Theory 50 (1987), 214-231.

[GK] V. Gorin, V. Kleptsyn, Universal objects of the infinite beta random matrix theory. Preprint, arXiv:2009.02006

[GM] V. Gorin, A.W. Marcus, Crystallization of random matrix orbits. Int. Math. Res. Notices 2020(3), 883913.

[GrM] P. Graczyk, J. Malecki, Strong solutions of non-colliding particle systems. Electron. J. Probab. 19 (2014), 21 pp.

[HT] U. Haagerup, S. Thorbjornsen, Random matrices with complex gaussian entries, Expo. Math. 21 (2003), 293-337.

[KM1] M. Kornyik, Gy. Michaletzky, Wigner matrices, the moments of Hermite polynomials and the semicircle law. J. Approx. Theory 211 (2016), 29-41.

[KM2] M. Kornyik, Gy. Michaletzky, On the moments of roots of Laguerre-polynomials and the Marchenko-Pastur law. Ann. Univ. Sci. Budapest., Sect. Comp. 46 (2017), 137-151.

[KVW] M. Kornyik, M. Voit, J. Woerner, Some martingales associated with multivariate Bessel processes. Acta Math. Hung., to appear, arXiv:1908.11189

[Me] M. Mehta, Random matrices (3rd ed.), Elsevier/Academic Press, Amsterdam, 2004.

[Men] G. Menon, Lesser known miracles of Burgers equation, Acta Math. Sci. 32B (2012), 281294.

[NS] A. Nica, R. Speicher, Lectures on the Combinatorics of Free Probability Theory, Cambridge University Press, Cambridge, 2006.

[OP] F. Oravecz, D. Petz, On the eigenvalue distribution of some symmetric random matrices, Acta Sci. Math. 63 (1997), 383-395.

[P] P.E. Protter, Stochastic Integration and Differential Equations. A New Approach. Springer, Berlin, 2003.

[RS] L.C.G. Rogers, Z. Shi, Interacting Brownian particles and the Wigner law. Probab. Theory Rel. Fields 95 (1993), 555-570.

[RW] L.C.G. Rogers, D. Williams, Diffusions, Markov Processes and Martingales, Vol. 1 Foundations. Cambridge University Press, Cambridge, 2000.

[R1] M. Rösler, Generalized Hermite polynomials and the heat equation for Dunkl operators. Comm. Math. Phys. 192 (1998), 519-542.

[R2] M. Rösler, Dunkl operators: Theory and applications. In: Orthogonal polynomials and special functions, Leuven 2002, Lecture Notes in Math. 1817 (2003), pp. 93-135. Springer Verlag, Berlin.

[RV1] M. Rösler, M. Voit, Markov processes related with Dunkl operators. Adv. Appl. Math. 21 (1998), 575-643.

[RV2] M. Rösler, M. Voit, Dunkl theory, convolution algebras, and related Markov processes. In: P. Graczyk et al. (eds.), Harmonic and stochastic analysis of Dunkl processes. pp. 1-112. Hermann, Paris 2008.

[Sch] B. Schapira, The Heckman-Opdam Markov processes. Probab. Theory Rel. Fields 138 (2007), 495-519.

[St] W.A. Strauss, Partial Differential Equations: An Introduction. Wiley, 1992.

[Sz] G. Szegö, Orthogonal Polynomials. Colloquium Publications (American Mathematical Society), Providence, 1939.

[V] M. Voit, Central limit theorems for multivariate Bessel processes in the freezing regime. $J$. Approx. Theory 239 (2019), 210-231.

[VW1] M. Voit, J.H.C. Woerner, Functional central limit theorems for multivariate Bessel processes in the freezing regime. Stoch. Anal. Appl., https://doi.org/10.1080/07362994.2020.1786402, arXiv:1901.08390

[VW2] M. Voit, J.H.C. Woerner, The differential equations associated with Calogero-MoserSutherland particle models in the freezing regime. Preprint 2019, arXiv:1910.07888

Fakultät Mathematik, Technische Universität Dortmund, Vogelpothsweg 87, D-44221 Dortmund, Germany

E-mail address: michael.voit@math.tu-dortmund.de, jeannette.woerner@math.tu-dortmund.de 